\title{
Spatial clustering in the ESO-Sculptor survey: two-point correlation functions by galaxy type at redshifts $0.1-0.5^{\star, \star \star}$
}

\author{
V. de Lapparent ${ }^{1}$ and E. Slezak ${ }^{2}$ \\ ${ }^{1}$ Institut d'Astrophysique de Paris, UMR 7095 CNRS, Univ. Pierre et Marie Curie, 98bis boulevard Arago, 75014 Paris, France \\ e-mail: lapparen@iap.fr \\ 2 Observatoire de la Côte d'Azur, BP 4229, 06304 Nice Cedex 4, France \\ e-mail: Eric.Slezak@oca.eu
}

Received 23 January 2007 / Accepted 4 June 2007

\begin{abstract}
Context. Galaxy clustering shows segregation effects with galaxy type, color and luminosity, which bring clues on the relationship with the underlying density field.

Aims. We explore these effects among the populations of giant and dwarf galaxies detected in the ESO-Sculptor survey.

Methods. We calculate the spatial two-point auto and cross-correlation functions for the 765 galaxies with $R_{\mathrm{c}} \leq 21.5$ and $0.1 \leq z \leq$ 0.51 and for subsets by spectral type and luminosity.

Results. At separation of $0.3 h^{-1} \mathrm{Mpc}$, pairs of early-type galaxies dominate the clustering over all the other types of pairs. At intermediate scales, $0.3-5 h^{-1} \mathrm{Mpc}$, mixed pairs of dwarf and giant galaxies contribute equally as pairs of giant galaxies, whereas the latter dominate at $\simeq 10 \mathrm{~h}^{-1} \mathrm{Mpc}$. Moreover, the correlation functions per galaxy type display the expected transition between the 1-halo and 2-halo regimes in the scenario of hierarchical merging of dark matter halos. The 1-halo component of the early-type galaxies largely outdoes that for the late spiral galaxies, and that for the dwarf galaxies is intermediate between both. In contrast, the 2-halo component of the early-type galaxies and late spiral galaxies are comparable, whereas that for the dwarf galaxies is consistent with null clustering.

Conclusions. We link the clustering segregation of the early-type and late spiral galaxies to their spatial distribution within the underlying dark matter halos. The early-type galaxies are preferentially located near the centers of the most massive halos, whereas late spiral galaxies tend to occupy their outskirts or the centers of less massive halos. This appears to be independent of luminosity for the early-type galaxies, whereas faint late spiral galaxies might reside in less dense regions than their bright analogs. The present analysis also unveils unprecedented results on the contribution from dwarf galaxies: at the scale at which they significantly cluster inside the halos $\left(\leq 0.3 h^{-1} \mathrm{Mpc}\right)$, they are poorly mixed with the late spiral galaxies, and appear preferentially as satellites of early-type galaxies.
\end{abstract}

Key words. surveys - galaxies: distances and redshifts - cosmology: large-scale structure of Universe galaxies: elliptical and lenticular, $\mathrm{cD}$ - galaxies: spiral - galaxies: dwarf

\section{Introduction}

The two-point correlation function is a fundamental statistic for characterizing the galaxy distribution. It partly quantifies the visual impression of clustering provided by the redshift maps, and subsequently allows one to perform direct comparison with the theoretical predictions. One of the issues is to determine how galaxies trace the underlying mass distribution, and whether and how this is related to their internal properties. This in turn can provide crucial information on how galaxies have formed and evolved until now.

Here we use the ESO-Sculptor Survey (hereafter ESS; de Lapparent et al. 2003) to statistically characterize the largescale clustering of galaxies at $z \lesssim 0.5$, and to examine its dependence on galaxy type. The ESS provides a nearly complete redshift survey of galaxies at $z \lesssim 0.5$ over a contiguous area of the sky (Bellanger et al. 1995), supplemented by CCD-based photometry (Arnouts et al. 1997) and a template-free spectral

* Based on observations collected at the European Southern Observatory (ESO), La Silla, Chile.

$\star \star$ All appendices are only available in electronic form at http://www . aanda. org classification (Galaz \& de Lapparent 1998). In agreement with the other existing redshift surveys to smaller or similar distances (de Lapparent et al. 1986; Shectman et al. 1996; Small et al. 1997; Colless et al. 2001; Zehavi et al. 2002), the ESS redshift map reveals a highly structured cell-like distribution out to $z \sim 0.5$ in which numerous sharp walls or filaments alternate with regions devoid of galaxies on a typical scale of $\sim 25 h^{-1}$ Mpc (Bellanger \& de Lapparent 1995). The deep pencilbeam geometry of the survey is characterized by a long line-ofsight of $1300 h^{-1} \mathrm{Mpc}$, and a transverse extent of $\sim 11 h^{-1} \mathrm{Mpc}$ at $z=0.3$, corresponding to $\sim 3$ correlation lengths (quoted scales are in comoving coordinates). The ESS therefore provides a sufficiently large sample for performing a useful two-point correlation analysis.

Using the ESS spectral classification and the corresponding luminosity functions per galaxy class (de Lapparent et al. 2003), we examine the variations in the ESS clustering as a function of galaxy type. Various surveys have detected the stronger clustering of early-type/red galaxies over late-type/blue galaxies at redshifts $z \lesssim 0.1$ (Loveday et al. 1999; Giuricin et al. 2001; Norberg et al. 2002; Magliocchetti \& Porciani 2003; Zehavi et al. 2005), and at higher redshifts $z \gtrsim 0.5$ (Shepherd et al. 2001; 
Phleps \& Meisenheimer 2003; Coil et al. 2004; Meneux et al. 2006). Further details were obtained by Li et al. (2006) from low redshift galaxies, whose analysis shows that the observed clustering differences between red and blue galaxies, namely a higher amplitude and steeper slope than for blue galaxies, are largest at small scales and for low mass galaxies; the authors also measure the same clustering segregation effects when considering galaxy age as traced by the $4000 \AA$ break strength, instead of galaxy color. To examine in further details the relationship between galaxy type and clustering, we propose here a new approach based on the separation of the giant and dwarf galaxies. We also measure the cross-correlation of the various samples, which provides complementary clues on the relative distribution of the different galaxy types.

The paper is organized as follows. Section 2 reviews the characteristics of the ESS galaxy redshift survey, defines the sub-samples used in the present analysis, and describes the luminosity functions used for calculating the selection functions. In Sect. 3, we evaluate the various sources of random and systematic errors. Results on the redshift space auto-correlation function $\xi(s)$ for the full ESS sample and the sub-samples by galaxy types are given in Sect. 4 . The correlation as a function of projected separation $w\left(r_{\mathrm{p}}\right)$ for the various ESS samples are described in Sect. 5, along with the cross-correlation functions between the different galaxy types. The auto and cross-correlation functions are then interpreted in Sect. 6 in terms of the occupation of the dark matter halos by the different galaxy types. In Sect. 7, we compare our results on $w\left(r_{\mathrm{p}}\right)$ to those from the other existing redshift surveys. Finally, Sect. 8 summarizes our conclusions and Sect. 9 discusses them in view of other existing results on galaxy clustering. In the Appendix, we describe the estimators which we use for calculating the two-point correlation functions, and we address the issues of the weighting scheme, the normalization of the correlation function, and the estimation of the mean density.

Throughout the present analysis, we assume a flat Universe with, at the present epoch, a scale parameter $H_{0}=$ $100 \mathrm{hm} \mathrm{s}^{-1} \mathrm{Mpc}^{-1}$, a matter density $\Omega_{\mathrm{m}}=0.3$ and a cosmological energy density $\Omega_{\Lambda}=0.7$ (Riess et al. 1998; Perlmutter et al. 1999; Phillips et al. 2001; Tonry et al. 2003). All absolute absolute magnitude are defined modulo $+5 \log h$.

\section{The ESO-sculptor survey}

The ESS covers a rectangular area of $0.37 \mathrm{deg}^{2}$ defined as a thin strip of $1.53^{\circ} \times 0.24^{\circ}$ near the south Galactic pole $\left(b_{\mathrm{II}} \sim\right.$ $-83^{\circ}$ ) and centered at $0^{\mathrm{h}} 22.5^{\mathrm{m}},-30.1^{\circ}$ (2000) in the Sculptor constellation. The observations were performed using the New Technology Telescope (NTT) and the $3.6 \mathrm{~m}$ telescope at the European Southern Observatory (ESO). The photometric catalogue is based on CCD multicolor imaging in the JohnsonCousins $B V R_{\mathrm{c}}$ system, and contains nearly 13000 galaxies to $V \simeq 24$ (Arnouts et al. 1997). The spectroscopic survey provides flux-calibrated spectra and redshifts (with an rms "external" uncertainty $\sigma(z) \sim 0.00055)$ for $\sim 600$ galaxies with $R_{\mathrm{c}} \leq 20.5$, within a slightly smaller field of $\sim 0.25 \mathrm{deg}^{2}=1.02^{\circ} \times 0.24^{\circ}$ (Bellanger et al. 1995). The $R_{\mathrm{c}} \leq 20.5$ sample has a $92 \%$ redshift completeness and its median redshift and effective depth are $z \simeq 0.3$ and $z \sim 0.5$, respectively. Additional redshifts for $\sim 250$ galaxies with $20.5<R_{\mathrm{c}} \leq 21.5$ were also measured in the same area, leading to a $52 \%$ redshift completeness to this fainter limit (see de Lapparent et al. 2003 for details).

The ESS spectroscopic catalogue was also used to perform a template-free spectral classification based on a principal component analysis of the flux-calibrated spectra, which yields a well-defined sequence parameterized continuously using 2 indices denoted $\delta$ and $\theta$ (Galaz \& de Lapparent 1998; de Lapparent et al. 2003): $\delta$ measures the shape of the continuum, hence the relative contribution from red and blue stellar populations, whereas the departures of $\theta$ from the sequence measure the strength of the nebular emission lines, hence the current star formation rate. Comparison with the Kennicutt templates (Kennicutt 1992) shows that the $\delta-\theta$ sequence is strongly related to the Hubble morphological type (see also Folkes et al. 1996; Bromley et al. 1998; Baldi et al. 2001), and provides a better estimate of the galaxy Hubble type than any color information (as used for example in Lilly et al. 1995; Lin et al. 1999).

The ESS catalogue is also complemented by precise typedependent K-corrections derived from the joint use of the spectral classification and the PEGASE spectrophotometric models (Fioc \& Rocca-Volmerange 1997). These in turn yield absolute magnitudes in the rest-frame filter bands (Johnson-Cousins $B V R_{\mathrm{c}}$ ), from which de Lapparent et al. (2003) have derived detailed luminosity functions as a function of spectral type. Here, we make use of these various parameters and characteristics of the ESS survey, to measure the two-point correlation functions.

\subsection{The galaxy samples}

The ESS spectroscopic sample was selected in $R_{\mathrm{c}}$ magnitude, and is thus most complete in this band, whereas the $V$ and $B$ samples suffer color-related selection effects at faint magnitudes (see de Lapparent et al. 2003). The present analysis is therefore based on the $R_{\mathrm{c}} \leq 21.5$ redshift sample, and all the quoted absolute magnitudes are in this band. In order to use only galaxies with a redshift value unaffected by peculiar motions within the local group, we omit in all samples the few ESS galaxies with $z \leq 0.1$. We also reject distant galaxies with $z \geq 0.51$ : at these redshifts, the selection function becomes of the order of $10 \%$ and decreases steeply, which causes a very sparse sampling of the large-scale structures. The bounding redshifts 0.1 and 0.51 correspond to comoving distances

$r_{\min }=284.33 h^{-1} \mathrm{Mpc}$

$r_{\max }=1338.04 h^{-1} \mathrm{Mpc}$,

respectively. Within these redshift boundaries, the ESS contains 765 galaxies with a reliable redshift, which lie in the $R_{\mathrm{c}}$ absolute magnitude interval $-23 \leq M+5 \log h \leq-16$.

To examine the varying clustering properties of the ESS with galaxy type, we define various sub-samples by galaxy type and absolute luminosity. We first consider the 3 spectral classes defined by de Lapparent et al. (2003): early-type with $\delta \leq-5$, intermediate-type with $-5<\delta \leq 3$, and late-type with $3<\delta$. As shown in de Lapparent et al. (2003), projection of the Kennicutt (1992) templates onto the ESS classification space indicates that these 3 classes approximately correspond to the following mixes of giant morphological types: $\mathrm{E}+\mathrm{S} 0+\mathrm{Sa}$ in the early class; $\mathrm{Sb}+\mathrm{Sc}$ in the intermediate class; and $\mathrm{Sc}+\mathrm{Sd} / \mathrm{Sm}$ in the late class.

Furthermore, the analysis of the ESS luminosity functions suggests that the ESS intermediate-type and late-type classes also contain dwarf morphological populations (de Lapparent et al. 2003): (1) gas-poor dwarf galaxies which are classified as intermediate spectral type due to their intermediate color, and most likely include dwarf elliptical (dE) and dwarf lenticular (dS0) galaxies, together with their nucleated analogs (Grant et al. 2005); here, these objects are altogether denoted dE; (2) dwarf 
Table 1. Definition of the ESO-Sculptor survey sub-samples used for calculation of the two-point correlation function.

\begin{tabular}{|c|c|c|c|c|c|c|c|c|}
\hline $\begin{array}{l}\text { Sub-sample } \\
\text { (1) }\end{array}$ & $\begin{array}{l}\text { Redshift range } \\
(2)\end{array}$ & $\begin{array}{c}R_{\mathrm{c}} \text { absolute magnitude range } \\
\text { (3) }\end{array}$ & \multicolumn{4}{|c|}{$\begin{array}{l}\text { Spectral range } \\
(4)\end{array}$} & \multirow[t]{2}{*}{$\begin{array}{l}N_{\mathrm{d}} \\
(5) \\
\end{array}$} & \multirow[t]{2}{*}{$\begin{array}{l}\langle\delta\rangle \\
(6) \\
\end{array}$} \\
\hline all galaxies: & & & & & $\leq$ & & & \\
\hline - with the over-density & ] $0.1 ; 0.51]$ & $-23.0 \leq M-5 \log h \leq-16.0$ & -20 & $<\delta$ & $\leq 2$ & 20 & 765 & -0.53 \\
\hline - without the over-density & ] $0.1 ; 0.51]-[0$. & $-23.0 \leq M-5 \log h \leq-16.0$ & -20 & $<\delta$ & $\leq 2$ & 20 & 654 & -0.09 \\
\hline - without the under-density & ] $0.1 ; 0.51]-[$ & $-23.0 \leq M-5 \log h \leq-16.0$ & -20 & $<\delta$ & $\leq 2$ & 20 & 709 & -0.44 \\
\hline early-type: & & & & $<$ & $\leq$ & & & \\
\hline - with the over-density & ]0.1;0.51] & $-23.0 \leq M-5 \log h \leq-16.0$ & -20 & $<\delta$ & $\leq-$ & -5 & 274 & -8.46 \\
\hline - bright early-type & $0.1 ; 0.51]$ & $-23.0 \leq M-5 \log h \leq-21.14$ & -20 & $<\delta$ & $\leq-$ & -5 & 137 & -8.63 \\
\hline - faint early-type & $0.1 ; 0.51]$ & $4 \leq M-5 \log h \leq-16.0$ & -20 & $<\delta$ & $\leq-$ & -5 & 137 & -8.29 \\
\hline - without the over-density & ] $0.1 ; 0.51]-[0.41 ; 0.44]$ & $-23.0 \leq M-5 \log h \leq-16.0$ & -20 & $<\delta$ & $\leq-$ & -5 & 218 & -8.43 \\
\hline - bright early-type & $; 0.51]-[0.41 ; 0$. & $-23.0 \leq M-5 \log h \leq-21.14$ & -20 & $<\delta$ & $\leq-$ & -5 & 97 & -8.45 \\
\hline - faint early-type & $; 0.51]-[0.41 ; 0.44]$ & $-21.14 \leq M-5 \log h \leq-16.0$ & -20 & $<\delta$ & $\leq-$ & -5 & 121 & -8.41 \\
\hline - without the under-density & $101 \cdot 051]-[0$ & $-23.0 \leq M-5$ & -20 & $<\delta$ & $\leq-$ & -5 & 245 & -8.45 \\
\hline intermediate-type: & & & & & $\leq$ & & & \\
\hline - with the ove & ] $0.1 ; 0.51]$ & $-23.0 \leq M-5 \log h \leq-16.0$ & -5 & $<\delta$ & & 3 & 240 & -1.04 \\
\hline - bright interme & ] $0.1 ; 0$ & $-23 .($ & -5 & $<\delta$ & $\leq$ & 3 & 207 & -1.15 \\
\hline - without the or & ] $0.1 ; 0.51]-[($ & -23 & -5 & $<\delta$ & $\leq$ & 3 & 206 & -1.03 \\
\hline - bright intermediate-type & $0.51]-[($ & -23 . & -5 & $<\delta$ & $\leq$ & 3 & 173 & -1.16 \\
\hline - without the under-density & ] $0.1 ; 0.51]-[0.2$ & $-23.0 \leq M-5 \log h \leq$ & -5 & $<\delta$ & $\leq$ & 3 & 231 & -1.02 \\
\hline late-type: & & & & & $\leq$ & & & \\
\hline - with the over-density & ] $0.1 ; 0.51]$ & $-23.0 \leq M-5 \log h \leq-19.30 /-19.57$ & 3 & $<\delta$ & $\leq 2$ & 20 & 251 & +8.61 \\
\hline - bright late-type & & -23 . & 3 & $<\delta$ & $\leq$ & 20 & 125 & +7.53 \\
\hline - without the over-density & ]0. & -23 & 3 & $<\delta$ & $\leq$ & 20 & 230 & +8.66 \\
\hline - bright late- $t$ & & -23. & 3 & $<\delta$ & $\leq$ & 20 & 106 & +7.53 \\
\hline - without the under-density & ] $0.1,0.01]-[0.04,0.59]$ & $-23.0 \leq M-5 \log h \leq-16.0$ & 3 & $<\delta$ & $\leq 2$ & 20 & 233 & +8.55 \\
\hline late spiral galaxies: & & & & & & & & \\
\hline - with & ] $0.1 ; 0.51]$ & $-23.0 \leq M-5 \log h \leq-19.30 /-19.57$ & -5 & $<\delta$ & & 20 & 332 & +2.11 \\
\hline - bright late spiral & ] $0.1 ; 0.5$ & $-23.0 \leq M-5 \log h \leq-20.56$ & -5 & $<\delta$ & $\leq$ & & 166 & +0.65 \\
\hline - faint late spiral & $0.1 ; 0.5$ & $-20.56 \leq M-5 \log h \leq-19.30 /-19.57$ & -5 & $<\delta$ & $\leq$ & 20 & 166 & +3.59 \\
\hline - without the over-density & ] $0.1 ; 0.51]-[0.41 ; 0.44]$ & $-23.0 \leq M-5 \log h \leq-19.30 /-19.57$ & -5 & $<\delta$ & $\leq$ & 20 & 279 & +2.14 \\
\hline - bright late spiral & ] $0.1 ; 0.51]-[($ & $-23.0 \leq M-5 \log h \leq-20.56$ & -5 & $<\delta$ & $\leq$ & 20 & 127 & +0.65 \\
\hline - faint late spiral & $0.51]-[0.41,0.44]$ & $-20.56 \leq M-5 \log h \leq-19.30 /-19.57$ & -5 & $<\delta$ & $\leq$ & 20 & 152 & +3.39 \\
\hline dwarf galaxies: & & & & & $\leq$ & & & \\
\hline - with the over-density & ] $0.1 ; 0.51]$ & $-19.30 /-19.57 \leq M-5 \log h \leq-16.0$ & -5 & $<\delta$ & $\leq$ & 20 & 159 & +7.59 \\
\hline - faint intermediate-type & ] $0.1 ; 0.51]$ & $-19.30 \leq M-5 \log h \leq-16.0$ & -5 & $<\delta$ & $\leq$ & & 33 & -0.37 \\
\hline - faint late-type & ] $0.1 ; 0.51]$ & $-19.57 \leq M-5 \log h \leq-16.0$ & 3 & $<\delta$ & 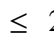 & 20 & 126 & +9.68 \\
\hline
\end{tabular}

Definition of columns: (1) identification of the sub-sample; (2) redshift interval; (3) interval of absolute magnitude; (4) interval of spectral index $\delta$; (5) number of galaxies in the sub-sample; (6) average spectral type $\delta$ for the sub-sample.

irregular (dI) galaxies, which are detected in the late spectral class due to their richer gas content, hence blue colors.

In the hierarchical scenario of galaxy formation, variations in the clustering properties of high mass and low mass galaxies are predicted (Pearce et al. 2001; Yang et al. 2004), which lead to the expectation of variations in the clustering properties of giant and dwarf galaxies. To separate the giant and dwarf galaxy populations which are mixed within the intermediate-type and late-type ESS spectral classes, we take advantage of their respective relative contribution at the bright and faint ends of the corresponding luminosity functions (see Fig. 11 in de Lapparent et al. 2003; or Fig. 1 of de Lapparent et al. 2004): we apply an $R_{\mathrm{c}}$ absolute luminosity cut at $M\left(R_{\mathrm{c}}\right)=-19.3$ for the intermediatetype galaxies, and at $M\left(R_{\mathrm{c}}\right)=-19.57$ for the late-type galaxies. We then merge the bright, resp. faint, populations of both spectral classes, to built 2 samples which are expected to include essentially:

- late spiral galaxies: $\mathrm{Sb}+\mathrm{Sc}+\mathrm{Sd} / \mathrm{Sm}$;

- dwarf galaxies: $\mathrm{dE}+\mathrm{dI}$.

To examine the dependence of the two-point correlation function on the absolute luminosity, we further divide the giant galaxy classes into "bright" and "faint" sub-samples defined by the median $R_{\mathrm{c}}$ absolute magnitude of the sample. Moreover, because the ESS exhibits a marked over-dense region in the interval $0.41 \leq z<0.44$ which causes a strengthening of the clustering signal (see Sect. 4.2), we also consider the sub-samples by galaxy type after removal of all galaxies in that particular redshift interval. At last, for further testing the sensitivity of the correlation function to cosmic variance, we also exclude the underdense region defined by $0.34 \leq z \leq 0.39$ (see Sect. 4.3). The characteristics of these various sub-samples are listed in Table 1.

\subsection{The luminosity functions}

For the luminosity function $\phi(M)$, we use the composite luminosity functions proposed by de Lapparent et al. (2003): a twowing Gaussian function for the early-type galaxies, and the sum of a Gaussian function and a Schechter (1976) function for the intermediate-type and late-type galaxies. These composite fits are motivated by their better adjustment to the ESS luminosity functions than pure Schechter functions, and by their good agreement with the luminosity functions per galaxy type measured locally (Jerjen \& Tammann 1997; see de Lapparent et al. 2003 for details). The composite fits of the ESS luminosity functions also confirm the morphological content of the ESS spectral classes in terms of giant galaxies and dwarf galaxies. 
Table 2. Parameters of the Gaussian and Schechter components of the composite luminosity functions fitted to the ESO-sculptor spectral classes at $R_{\mathrm{c}} \leq 21.5$.

\begin{tabular}{lccc}
\hline \hline Luminosity function parameters & Early-type & Intermediate-type & Late -type \\
\hline Gaussian component: & $\mathrm{E}+\mathrm{S} 0+\mathrm{Sa}$ & $\mathrm{Sb}+\mathrm{Sc}$ & $\mathrm{Sc}+\mathrm{Sd} / \mathrm{Sm}$ \\
Morphol. $\operatorname{content}$ & $-20.87 \pm 0.23$ & $-20.27 \pm 0.21$ & $-19.16 \pm 0.29$ \\
$M_{0}-5 \log h$ & $0.84 \pm 0.24 / 1.37 \pm 0.36$ & $0.91 \pm 0.18$ & $0.97 \pm 0.13$ \\
$\sigma$ & 0.00333 & 0.00326 & $0.00194[1+3.51(z-0.15)]$ \\
$\phi_{0}$ & & $\mathrm{dE}$ & $\mathrm{dI}$ \\
\hline Schechter component: & $-19.28 \pm 0.37$ & $-18.12 \pm 0.22$ \\
Morphol. $\operatorname{content}$ & $-1.53 \pm 0.33$ & -0.30 \\
$M^{*}-5 \log h$ & 0.00426 & $0.02106[1+3.51(z-0.15)]$ \\
$\alpha$ & & \\
$\phi^{*}$ & &
\end{tabular}

Table 2 lists the various giant and dwarf components of the luminosity functions for the 3 spectral classes and the corresponding parameters. The two-wing Gaussian luminosity function for the early-type galaxies is parameterized as

$$
\begin{aligned}
\phi(M) \mathrm{d} M & =\phi_{0} \mathrm{e}^{-\left(M_{0}-M\right)^{2} / 2 \sigma_{a}^{2}} \mathrm{~d} M \text { for } M \leq M_{0} \\
& =\phi_{0} \mathrm{e}^{-\left(M_{0}-M\right)^{2} / 2 \sigma_{b}^{2}} \mathrm{~d} M \text { for } M \geq M_{0}
\end{aligned}
$$

where $M_{0}$ is the peak magnitude, and $\sigma_{a}$ and $\sigma_{b}$ are the dispersion values for the 2 wings. The Gaussian component of the intermediate-type and late-type luminosity functions is parameterized as

$\phi(M) \mathrm{d} M=\phi_{0} \mathrm{e}^{-\left(M_{0}-M\right)^{2} / 2 \sigma^{2}} \mathrm{~d} M$,

where $M_{0}$ and $\sigma$ are the peak and rms dispersion respectively. The Schechter (1976) component of the intermediate-type and late-type luminosity functions is parameterized as

$\phi(M) \mathrm{d} M=0.4 \ln 10 \phi^{*} \mathrm{e}^{-X} X^{\alpha+1} \mathrm{~d} M$

with

$X \equiv \frac{L}{L^{*}}=10^{0.4\left(M^{*}-M\right)}$

where $M^{*}$ is the characteristic magnitude, and $\alpha+1$ the "faintend slope". For the late spiral and dwarf sub-samples, we use the bright and faint parts resp. of the luminosity functions by spectral type (see Table 1).

The parameters listed in Table 2 are those derived by de Lapparent et al. (2004) from the $R_{\mathrm{c}} \leq 21.5$ sample. For the early-type and intermediate-type classes, the listed values of $\phi_{0}$ are those listed as $\Phi_{1}(0.51)$ in Table 2 of de Lapparent et al. (2004), derived using an "equal pair" weighting for the mean density estimator (see Eqs. (F.1) and (D.1)); they thus yield a total expected number of galaxies over the redshift interval $0.1-0.51$ for a homogeneous distribution which is equal to the observed number of galaxies in each spectral class. For the late-type class, we use (and list here in Table 2) the parameterization $\Phi_{1}(z)$ provided in Table 2 of de Lapparent et al. (2004), which reflects the marked evolution of the amplitudes $\phi_{0}$ and $\phi^{*}$ for the ESS late-type galaxies. This evolution could also be due to pure luminosity evolution, but the available data do not allow us to discriminate between the 2 effects (de Lapparent et al. 2004). Whatever the nature of this evolution, the parameterized amplitude evolution listed in Table 2 does allow us to estimate the selection function (Appendix C) required for calculating correlation functions.
For the intermediate-type and late-type class, the values of $\phi^{*}$ are derived from the values of $\phi_{0}$ using the ratios

$$
\begin{aligned}
& \frac{\phi_{0}}{0.4 \ln 10 \phi^{*}}=0.83 \text { for intermediate-type } \\
& \frac{\phi_{0}}{0.4 \ln 10 \phi^{*}}=0.10 \text { for late-type }
\end{aligned}
$$

also provided by de Lapparent et al. (2004, see their Table 1).

These various luminosity functions allow us to derive the corresponding selection functions affecting the ESS sub-samples (see Sect. C). These yield the expected redshift distribution for homogeneous samples, which are shown in Figs. 1 and 2 and are compared with the ESS observed distributions for each spectral class and galaxy type. To calculate the expected redshift distribution, we also use for each sub-sample the $K$-correction function $K(z, \delta)$ provided by de Lapparent et al. (2004) at the mean value of the spectral-type $\delta$ listed in the Table 1 . Note that comparison of the observed and expected distributions for the 3 spectral classes shown in Fig. 1 confirms the validity of the luminosity functions and amplitudes listed in Table 2, and in particular validates the parameterization of the amplitude evolution for the late-type galaxies.

\section{Computing the correlation function and its uncertainties}

The formalism for derivation of the comoving distances $r$ from the redshifts, for calculating the three different estimators of the redshift-space and projected correlation function (Davis-Peebles DP, Landy-Szalay LS, and Hamilton H), the various selection functions and the corresponding three weighting schemes $(\mathrm{J} 3$, "equal volume" EV, and "equal pair" EP), the normalization, and the mean density are defined in the Appendix. Because the variance in the estimates of $\xi(s)$ depends on the chosen statistical weights, it is useful to apply the three weighting schemes to each of the 3 estimators of the correlation function, and to cross-check the results; this is done in the Sect. 4. Here, as a preliminary, we describe and estimate the various uncertainties at play in the correlation function measurements.

\subsection{Statistical noise in $\xi(s)$}

From the experimental point of view, statistical error bars in $\xi(s)$ would be best determined from the ensemble error by splitting the survey into independent regions containing approximately 


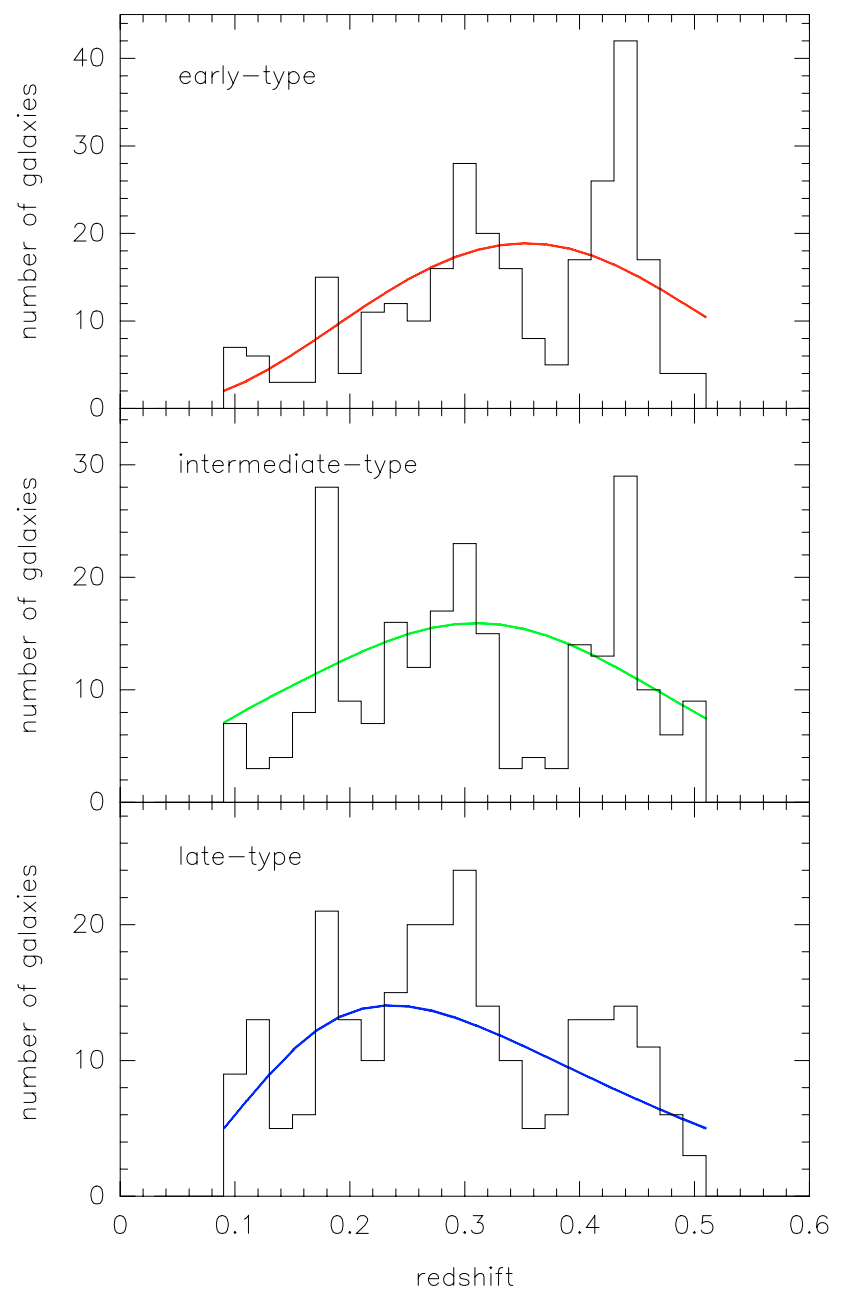

Fig. 1. Redshift histograms for the early-type (top panel), intermediatetype (middle panel), and late-type (bottom panel) spectral classes of the ESO-Sculptor survey, using a redshift bin of 0.02. The solid curves show the expected distributions for a homogeneous sample given the luminosity functions defined in Table 2, the magnitude limits and the angular coverage of the survey.

equal numbers of galaxies and taking the standard deviation of the correlation function estimates calculated in these sub-areas. Unfortunately, the size of the ESS redshift sample is too small to allow for such an approach. Here we consider both the Poisson error

$\sigma_{\text {Poiss }}(s)=[1+\xi(s)] D D(s)^{-1 / 2}$

and the bootstrap error $\sigma_{\text {boot }}(s)$, which is derived as the standard deviation in the estimates $\xi_{(k)}$ from 50 randomly data sets of $N_{\mathrm{d}}$ points obtained from the original $N_{\mathrm{d}}$ galaxies by re-sampling with replacement, without any correction for possible systematic biases (Ling et al. 1986). The Poisson error is an underestimate of the true uncertainty for correlated data but is appropriate in the regime of weak clustering, i.e. at large scales $\left(s \gtrsim 10 h^{-1} \mathrm{Mpc}\right)$. In contrast, the bootstrap error tends to overestimate the true error in over-dense regions by roughly a factor two, as shown by Fisher et al. (1994). In the following, we thus adopt the bootstrap error at all scales $s$ as the statistical random uncertainty in the measured values of $\xi(s)$ and $\xi\left(r_{\mathrm{p}}, \pi\right)$.

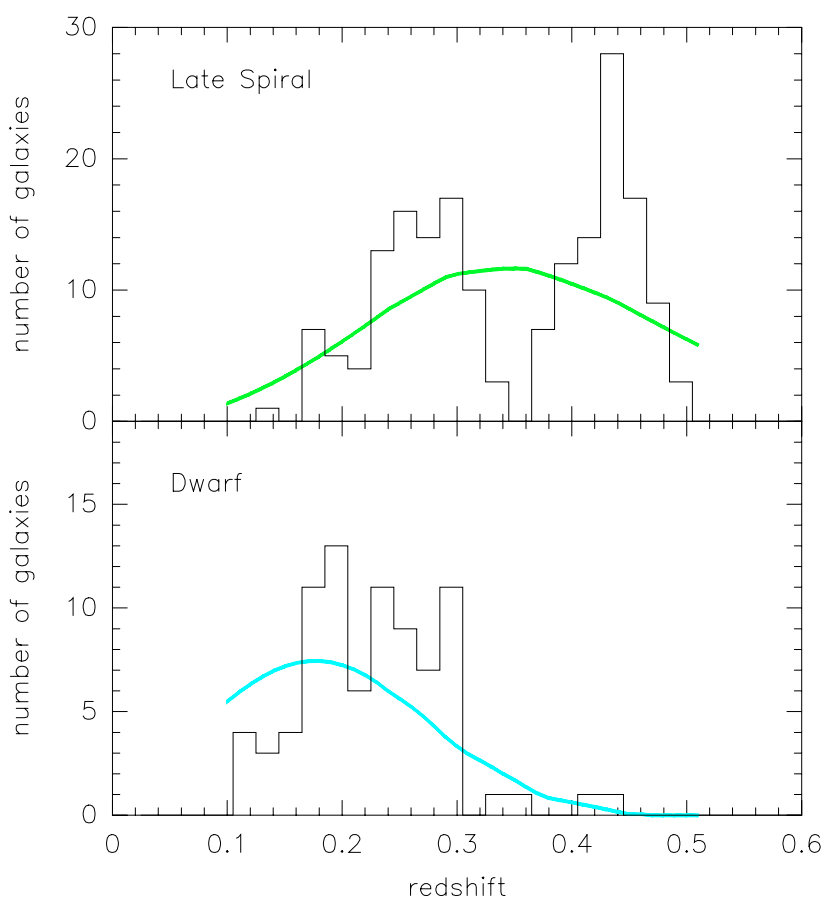

Fig. 2. Redshift histograms for the late spiral (top panel) and dwarf (bottom panel) sub-samples of the ESO-Sculptor survey (see Fig. 1 for details).

\subsection{Uncertainties from distances}

A priori, derivation of distances for the ESS galaxies requires to correct the ESS velocities for the motion within the Local Group (Yahil et al. 1977; Courteau \& van den Bergh 1999), the infall onto Virgo (Ramella et al. 1997; Ekholm et al. 2001), and the cosmic microwave background dipole (Smoot et al. 1991). We estimate the impact of these velocity corrections by calculating the difference in correction value for 4 imaginary points located at the extreme 4 "corners" or the ESS survey region. Each "corner" point is defined by the constant value of either one of the 2 coordinates RA (J2000), Dec (J2000) among

$\begin{array}{ll}\mathrm{RA}_{\text {min }}=0^{\mathrm{h}} 19.0^{\mathrm{m}} & \mathrm{RA}_{\max }=0^{\mathrm{h}} 23.5^{\mathrm{m}} \\ \mathrm{Dec}_{\text {min }}=-30^{\circ} 14^{\prime} & \operatorname{Dec}_{\text {max }}=-29^{\circ} 58^{\prime},\end{array}$

while the other coordinate takes the 2 possible values. The largest resulting velocity differences are $2.5 \mathrm{~km} \mathrm{~s}^{-1}$ for pairs of points in which only RA varies; $1.4 \mathrm{~km} \mathrm{~s}^{-1}$ for pairs of points in which only Dec varies. The largest velocity difference of $3.7 \mathrm{~km} \mathrm{~s}^{-1}$ is obtained for the pair of points with coordinates $\left(\mathrm{RA}_{\min }, \mathrm{Dec}_{\max }\right)$ and $\left(\mathrm{RA}_{\max }\right.$, Dec $\left.c_{\min }\right)$. These values are negligeable compared to the ESS rms uncertainty in the velocities of $\sigma(v) \sim 165 \mathrm{~km} \mathrm{~s}^{-1}$. We thus neglect the effects of the 3 mentioned systematic motions in the estimation of distances.

The uncertainties in the ESS redshifts $(\sigma(z) \sim 0.00055)$ is also a source of random error in the measurement of the correlation function. To estimate its impact, we add to all galaxies in the ESS early-type sub-sample an additional dispersion in the redshift $\delta z$ defined by a Gaussian probability distribution centered at zero and with an rms deviation $\sigma(z) \sim 0.00055$. We repeat this procedure 30 times, and measure an rms dispersion in $\xi_{\mathrm{LS}}(s)$ at scales $1<s<15 h^{-1} \mathrm{Mpc}$ which is 2 to 3 times smaller than the bootstrap uncertainty. Similar results are obtained using the intermediate-type and late-type sub-samples. We thus neglect this additional source of random error. 


\subsection{Uncertainties from the luminosity function}

As far as systematic errors are concerned, a dominant contribution to the correlation function may be the uncertainty in the selection function (Appendix $\mathrm{C}$ ), which results from the uncertainties in the luminosity function. As shown by Peebles (1980), the quadratic $\mathrm{H}$ estimator (Eq. (B.6)) is more affected than the DP and LS estimators (Eqs. (B.5) and (B.7); see Sect. B). For power-law correlation functions (Eq. (D.4)), such biases have more impact on the correlation length $s_{0}$ than on the power-law index $\gamma$. As described in Sect. 2.2 and Table 2, five luminosity functions are involved in the computation according to the considered spectral class. In principle, any derivation of $s_{0}$ and $\gamma$ should be repeated by varying the selection function parameters along the six principal axes of its error ellipsoid, and the scatter among the results added in quadrature to their statistical uncertainties. In practise, we change the value of $M_{0}$ for the early-type and intermediate-type luminosity functions, and the value of the Schechter slope $\alpha$ for the intermediate-type and latetype luminosity functions by plus or minus their rms uncertainty (listed in Table 2). The resulting systematic shifts in $\xi_{\mathrm{LS}}(s)$ for $1 \leq s \leq 5 h^{-1} \mathrm{Mpc}$ are $\pm 4.2 \%$ and $\pm 8.6 \%$ when changing $M_{0}$ for the early-type and intermediate-type samples resp.; and ${ }_{-3.5 \%}^{+4.0 \%}$ and ${ }_{5.3 \%}^{+9.0 \%}$ when changing $\alpha$ for the intermediate-type and latetype samples respectively; these various shifts are $\sim 4$ to 10 times smaller than the bootstrap errors for each sample.

\subsection{Uncertainties from the $J_{3}$ weighting}

Another source of uncertainty is the choice of the parameterization for $J_{3}(s)$ in the case of a $J_{3}$ weighting scheme (see Eq. (D.3)). We estimate this uncertainty by comparing $\xi_{\mathrm{LS}}(s)$ for the ESS early-type sub-sample with that using the following $J_{3}$ weighting parameterization

$J_{3}(s)=14.98 s^{1.4} h^{-3} \mathrm{Mpc}^{3}$ for $s \leq s_{\mathrm{c}}$,
$J_{3}(s)=1752 h^{-3} \mathrm{Mpc}^{3}$ for $s>s_{\mathrm{c}}$.

(with $s_{\mathrm{c}} \equiv 30 h^{-1} \mathrm{Mpc}$ ). This other parameterization of $J_{3}(s)$ is obtained from the power-law description of the correlation function (Eq. (D.4)) with a $10 \%$ increase of $s_{0}$ above the value given in Eq. (D.5). The relative change in $\xi_{\mathrm{LS}}(s)$ is then $\lesssim 1.1 \%$ at all scales, which is one to two orders of magnitude smaller than the bootstrap random uncertainties. Performing a similar test in which only the slope $\gamma$ of the power-law parameterization is increased by $10 \%$ over the value in Eq. (D.5) yields slightly larger variations in $\xi(s)(<2.2 \%)$. Decreasing either $s_{0}$ or $\gamma$ by similar amounts yields the corresponding opposite shifts in $\xi(s)$. The systematic uncertainties in $J_{3}(s)$ therefore have a very small impact on the error budget in $\xi(s)$.

\subsection{Cosmic bias}

The correlation function is also affected by a systematic bias called "cosmic bias", which is caused by using the observed density of galaxies in the sample for normalization of the number of pairs (see Appendix E). This results in an implicit normalization to zero of the integral of the correlation function over the survey volume. However, in finite samples, the two-point correlation function is positive out to scales of $\sim 20 \mathrm{~h}^{-1} \mathrm{Mpc}$, and the mean density of galaxies estimated from a sample of comparable scale is an over-estimate of the mean density of the Universe. The corresponding bias in the correlation function is then expected to be negative. In that case, the cosmic bias can be corrected for by an additive correction, called "integral constraint" (Ratcliffe et al. 1996; Brainerd et al. 1995). In contrast, for sample sizes of $\sim 100 \mathrm{~h}^{-1} \mathrm{Mpc}$ or larger, the presence of an under-density occupying a large volume may instead lead to an under-estimation of the mean density and a corresponding over-estimation of the correlation function.

To estimate the cosmic bias for the ESS, we vary by $\pm 4 \%$ (which corresponds to $\sqrt{N} / N \simeq 0.036$, where $N=765$ is the number of galaxies in the full ESS sample, see Table 1) the amplitude $\phi_{0}$ of the Gaussian component of the early-type, intermediate-type and late-type luminosity functions, and the amplitude $\phi^{*}$ of the Schechter components of the intermediatetype and late-type luminosity functions (see Table 2), and calculate the resulting $\xi(s)$ for the full ESS sample. The shift in $\xi(s)$ for the full ESS sample is $\lesssim 4 \%$ for $1 \leq s \leq 9 h^{-1} \mathrm{Mpc}$, which is 10 times smaller than the bootstrap error; the shift then increases at larger scales, taking its largest value at $s \simeq 14-28 h^{-1} \mathrm{Mpc}$, as it is comparable to the transverse extent of the survey.

Nevertheless, we show in Sect. 4.2 that a marked over-dense region of the ESS causes a larger systematic shift in the correlation function than the above estimated cosmic bias, and furthermore, this shift is in the opposite direction (an excess correlation). We thus choose to neglect the standard "cosmic bias", and instead, we evaluate the impact of this over-density onto the various measured correlation functions in Sects. 4.1, 4.2, 4.3, 5.1 and 5.3. In Sect. 5.2, we further discuss the role of this structure in terms of cosmic variance.

\section{The redshift-space correlation function $\xi(s)$}

\subsection{General behavior of $\xi(s)$}

Top panel of Fig. 3 shows the 9 combinations of the 3 estimators H, LS and DP (see Sect. B) and the 3 weighting schemes J3, EV and EP (see Sect. D) applied to the full ESS sample limited to $R_{\mathrm{c}} \leq 21.5,-23<M\left(R_{\mathrm{c}}\right)-5 \log h<-16$ and $0.10<z<0.51$ (765 objects, see Table 1). Comparison of the various estimators confirms that the $J 3$ weighting behaves as the EP weighting at small separation $s$, and as the EV weighting at large $s$. For a given weighting scheme and at scales $s \gtrsim 0.2 h^{-1} \mathrm{Mpc}$, the differences between the 3 estimators are significantly smaller than the error bars in each estimate. The bootstrap errors for the LS estimate of $\xi(s)$ are shown in Fig. 4; the bootstrap errors in the DP and $\mathrm{H}$ estimates are comparable to those in the LS estimate, but have a less stable behavior with varying separation. For this reason, and because the $J 3$ weighting is the minimum variance weighting and does not favor nearby nor distant pairs, we only show and examine in the following the LS estimate of $\xi(s)$ with $J 3$ weighting; note, however, that the results and conclusions of the article are unchanged using the other estimators and weighting schemes.

The various pairs of objects in top panel of Fig. 3 are counted in logarithmic bins of equal size with $\Delta \log s=0.1$. In the following, we adopt this bin size, as it allows one to probe the small scale regime $s \lesssim 1 h^{-1} \mathrm{Mpc}$. Note that even if $\xi(s)$ for the ESS is biased on these scales, $w\left(r_{\mathrm{p}}\right)$ can successfully be measured down to $s \simeq 0.2 h^{-1} \mathrm{Mpc}$ (see Sect. 5). We show in bottom panel of Fig. 3 that the overall behavior of $\xi(s)$ for the ESS is kept unchanged when shifting the bin set by half the bin size, or when using larger bin sizes with $\Delta \log s=0.15$ and $\Delta \log s=0.2$; similar conclusions are drawn for the correlation functions $\xi\left(r_{\mathrm{p}}, \pi\right)$ and $w\left(r_{\mathrm{p}}\right)$ considered in the following sections.

In Fig. 4, we show the LS estimate of $\xi(s)$ with $J 3$ weighting from the full ESS sample (labeled as " $0.10<z<0.51$ "), along 


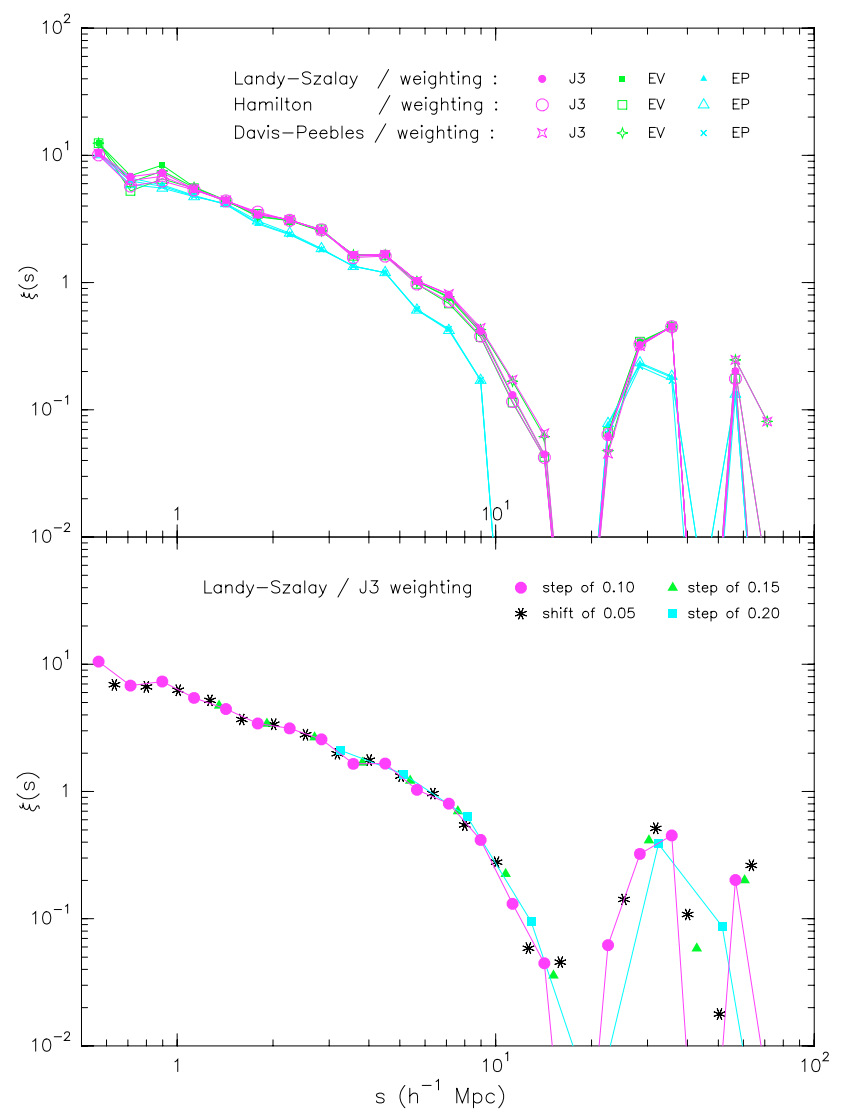

Fig. 3. The redshift-space correlation function $\xi(s)$ for all ESO-Sculptor galaxies with $0.10<z<0.51$. The top panel displays the result obtained with a bin size of $\Delta \log (s)=0.10$ for the 3 estimators Landy-Szalay (filled symbols), Hamilton (open symbols), Davis-Peebles (starred symbols), and the 3 weighting schemes J3 (magenta circles and diagonal stars), "equal volume" (denoted EV, green squares and stars) and "equal pair" (denoted EP, cyan triangles and cross). The bottom panel compares the Landy-Szalay estimate of $\xi(s)$ with a $J 3$-weighting for different values of the bin size: $\Delta \log (s)=0.10$ (magenta circles), $\Delta \log (s)=0.15$ (green triangles), $\Delta \log (s)=0.20$ (cyan squares); the bin size $\Delta \log (s)=0.10$ with a 0.05 shift in $\Delta \log (s)$ from the origin is also plotted (black asterisks). For sake of clarity, only two sets of points are connected by a solid line.

with its bootstrap errors; in top panel, $\xi(s)$ is in logarithmic scale, and in bottom panel, in linear scale. The redshift-space correlation function has the usual power-law behavior at small scales, and a smooth roll-off at $s \lesssim 10 h^{-1} \mathrm{Mpc}$. The adjustment of a power-law (see Eq. (D.4)) in the interval $0.5<s<5.0 h^{-1} \mathrm{Mpc}$ yields a correlation length and correlation slope of

$s_{0}=7.49 \pm 3.18 h^{-1} \mathrm{Mpc}, \quad \gamma=0.90 \pm 0.13$,

resp.; this power-law fit is shown as a solid line in both panels of Fig. 4.

Top panel of Fig. 4 shows that at larger scales, $s \sim$ $10 h^{-1} \mathrm{Mpc}, \xi(s)$ breaks down from the power-law fit. Then at $s \simeq 15 h^{-1} \mathrm{Mpc}$, the amplitude of the correlation function crosses zero, as seen in bottom panel of Fig. 4. $\xi(s)$ rises up again at scales $25 \lesssim s \lesssim 40 h^{-1} \mathrm{Mpc}$, and another peak occurs at $50<s<60 h^{-1} \mathrm{Mpc}$. This large-scale behavior is discussed in the next sub-sections.

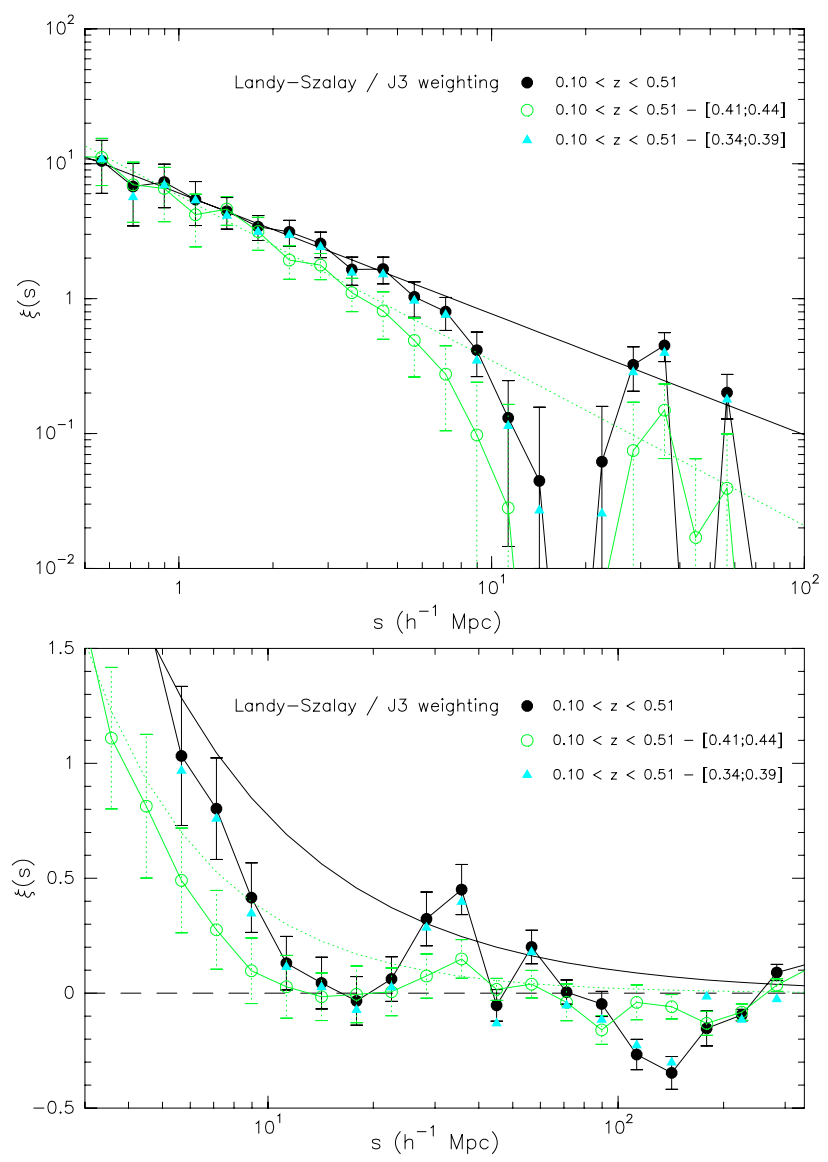

Fig. 4. The redshift-space correlation function $\xi(s)$ for the ESOSculptor galaxies, computed with the Landy-Szalay estimator using the minimum-variance $J_{3}$-weighting scheme for (i) all galaxies with $0.10<z<0.51$ (black filled circles); (ii) the sub-sample in which the over-density in the interval $0.41<z<0.44$ is removed (green open circles); (iii) the sub-sample in which the under-dense region in the interval $0.34<z<0.39$ is removed (cyan triangles). The bottom panel shows the same curves in linear scale, restricted to $s>3 h^{-1} \mathrm{Mpc}$. Both panels display $\xi(s)$ with a bin size $\Delta \log (s)=0.10$, and show the powerlaw model fitted to the full sample with $0.10<z<0.51$ in the interval $0.5<s<5.0 h^{-1} \mathrm{Mpc}$ (black solid line). The bootstrap error bars for the sub-sample without the under-density at $0.34<z<0.39$ are not shown, as they are similar to those for the full sample.

\subsection{Impact of the over-density at $0.41<z<0.44$}

Part of the deviations of $\xi(s)$ from null clustering at $s \gtrsim$ $10 h^{-1} \mathrm{Mpc}$ (seen in bottom panel of Fig. 4) appear to be due to the presence of a marked over-density in the redshift interval $0.41<z<0.44$. This structure is clearly seen in the redshiftcones of the survey shown in Fig. 5. It also appears in the redshift histograms shown in Fig. 1 as an integrated excess by nearly a factor 2 over the expected number for a homogeneous distribution (indicated by the solid lines) for the early-type and late-type galaxies.

The ESS over-dense region at $0.41<z<0.44$ has an impact on the redshift-space correlation function at both intermediate and large scales. Top panel of Fig. 4 shows that when removing the over-density, $\xi(s)$ shifts to lower amplitudes in the interval $0.5 \leq s \leq 5 h^{-1} \mathrm{Mpc}$, which results in a lower amplitude and steeper slope for the power-law fit:

$s_{0}=4.22 \pm 1.15 h^{-1} \mathrm{Mpc}, \quad \gamma=1.22 \pm 0.15$, 


\title{
ESO-Sculptor Survey
}

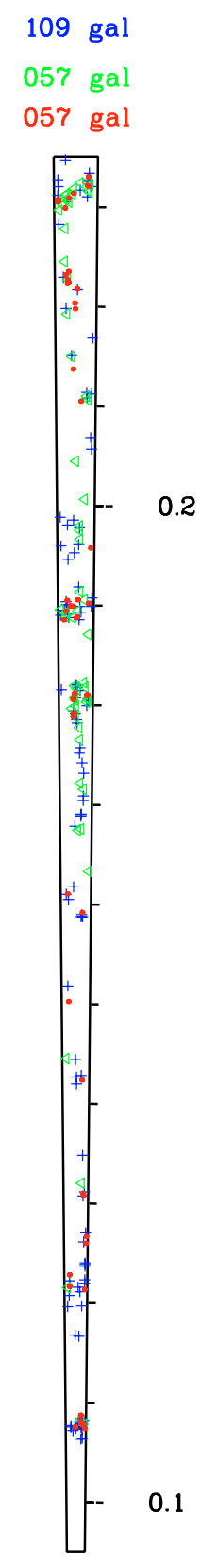

\author{
$046 \mathrm{gal}$ \\ 129 gal \\ $103 \mathrm{gal}$
}

005 gal

147 gal

$160 \mathrm{gal}$

dwarf

$116 \mathrm{gal}$

$333 \mathrm{gal}$

late Spiral

276 gal

early-type $\quad$
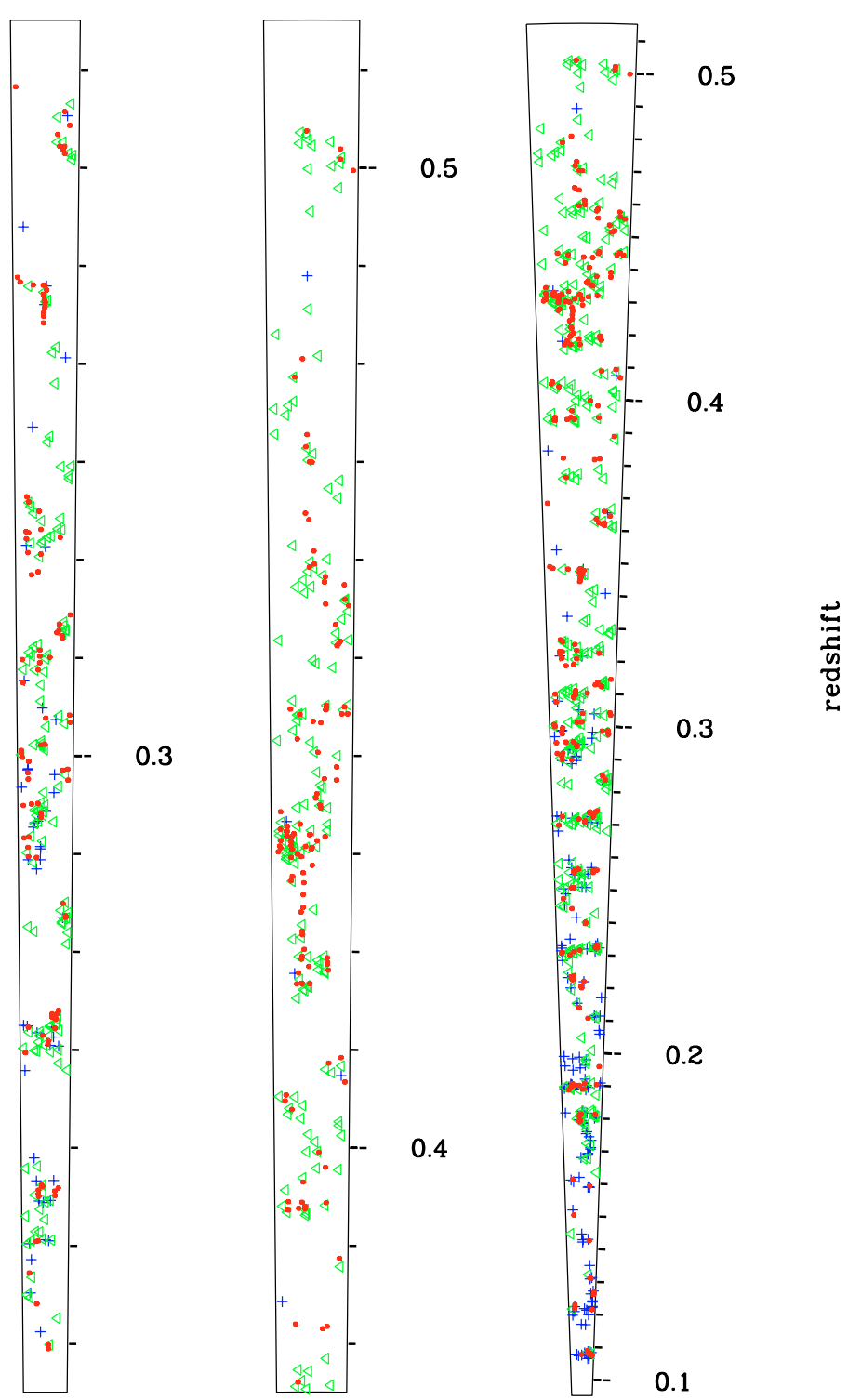

0.3

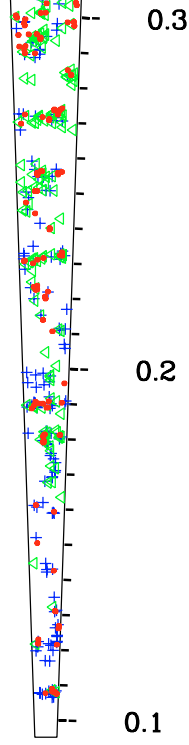

right ascension

Fig. 5. Redshift cone-diagrams for the ESO-Sculptor survey, truncated into 3 redshift intervals ( 3 left cones). A total number of 769 galaxies with $R \leq 21.5$ and reliable redshift in the interval $0.1 \leq z \leq 0.51$ are plotted in the full cone (to the right), which is stretched in angle by a factor 3 . Dwarf galaxies (blue crosses) are plotted first, then the late spiral galaxies (green triangles), and finally the early-type galaxies (red disks). These graphs show that the survey intercepts many large-scale structures, appearing as an alternation of voids and walls or filaments. The stronger clustering of the early-type galaxies over the late spiral and dwarf galaxies is also visible.

(measured for $0.5<s<5 h^{-1} \mathrm{Mpc}$ ). If the change in $\xi(s)$ was solely due to the change in the normalizing density, a higher amplitude of $\xi(s)$ would be expected when removing the galaxies in the $0.41<z<0.44$ interval, as this over-dense region tends to artificially increase the mean density of the sample, thus contributing to the cosmic bias (see Sect. 3.5). The decreasing amplitude of $\xi(s)$ at $s \gtrsim 2 h^{-1}$ Mpc implies that galaxies in the $0.41<z<0.44$ region have stronger clustering at medium and large scale than the average for the rest of the survey. Indeed, detailed examination of Fig. 5 indicates that the large-scale structure at $0.41<z<0.44$ is a dense collection of groups of galaxies extending over $\Delta z \sim 0.025$, that is $\sim 60 h^{-1} \mathrm{Mpc}$ in comoving distance. In Sect. 4.4 below, we show that both the early-type and late spiral galaxies contribute to the excess clustering in this region.

When the galaxies in the redshift interval $0.41<z<0.44$ are removed from the ESS full sample, the deviations of $\xi(s)$ from zero at scales $9 \lessgtr s \lesssim 150 h^{-1}$ Mpc (see bottom panel of Fig. 4) are reduced to less than the bootstrap uncertainty for most points. In particular, the second peak at $50<s<60 h^{-1} \mathrm{Mpc}$ 
becomes insignificant. The peak at $25 \lesssim s \lesssim 40 h^{-1} \mathrm{Mpc}$ is also significantly reduced to a "marginal" detection, as $\xi(s)$ deviates by less than twice the bootstrap error for $s \simeq 35 h^{-1} \mathrm{Mpc}$. One possible interpretation of the shift towards negative values of $\xi(s)$ at $s \sim 90 h^{-1} \mathrm{Mpc}$, when removing the over-density within $0.41<z<0.44$, could be the artificial anti-correlation thus created between this empty region and the foreground/background walls of galaxies: the difference in comoving distance between $z=0.41$ and $z=0.44$ is $\simeq 72 h^{-1} \mathrm{Mpc}$.

When removing a given redshift interval from an ESS sample, the selection function of each of the 3 spectral-type samples is assigned to zero in that interval, and no points are generated in that redshift interval of the random distributions. To check that the procedure does not introduces any bias, we also calculate $\xi(s)$ for the full ESS sample with all galaxies in the redshift interval $0.34<z<0.39$ removed: this region of the ESS has a similar volume as the region defined by $0.41<z<0.44$, and corresponds to an under-dense region in the ESS early-type and intermediate-type redshift histograms (Fig. 1). The resulting $\xi(s)$ with the $0.34<z<0.39$ interval removed is over-plotted in both panels of Fig. 4, and compared to that for the full ESS sample: it shows negligeable changes at all scales. Although the $0.34<z<0.39$ region is under-dense compared to the rest of the ESS, it has little impact on the two-point correlation function at all scales.

\subsection{Large-scale power in $\xi(s)$}

The excess in $\xi(s)$ at $25 \lesssim s \lesssim 40 h^{-1} \mathrm{Mpc}$, seen in bottom panel of Fig. 4, might also be due in part to the pencil-beam geometry of the ESS. The large-scale clustering of galaxies is characterized by walls and filaments which delineate large voids (de Lapparent et al. 1986; Shectman et al. 1996; Small et al. 1997; Colless et al. 2001; Zehavi et al. 2002). This results in an alternation of voids and narrow portions of walls or filaments intercepted at a wide range of angles with the line-of-sight of the survey, as one then expects few walls, and even fewer filaments, to be intercepted parallel to the line-of-sight, and thus to appear as an extended over-density along the line-of-sight (the over-density at $0.41<z<0.44$ may however be one of these rare occurrences). Then, the particular line-of-sight and limited volume sampled by the ESS may define a typical scale for the wall/filament separation, which would appear as excess signal in the two-point correlation.

A pair separation analysis provides quantitative evidence for the impact of the ESS pencil-beam geometry onto the detected large-scale clustering. Top panel of Fig. 6 shows the histogram of the galaxy pair separations in comoving distance for the ESS full sample. This distribution exhibits numerous peaks which seem to define a preferred scale. The bottom panel of Fig. 6 shows the "periodogram" obtained by a spectral density analysis of the pair separation distribution from which the continuum has been subtracted. A marked peak occurs at $34 h^{-1} \operatorname{Mpc}(v=0.0295)$, which indicates an increased probability of having pairs of ESS galaxies separated by this scale. This in turn explains the excess signal in $\xi(s)$ at $25 \lesssim s \lesssim 40 h^{-1} \mathrm{Mpc}$ (bottom panel of Fig. 4). Note that this scale corresponds to the mean interval picked up by the eye between adjacent walls/filaments in the cone diagram of the ESS (Fig. 5): $34 h^{-1} \mathrm{Mpc}$ corresponds to $\Delta z=0.013$ at $z=0.3$, that is $30 \%$ larger than the tick mark separation. When the over-dense region with $0.41<z<0.44$ is removed from the ESS, the results of the pair separation analysis remain; the peak at $34 h^{-1} \mathrm{Mpc}$ in the periodogram becomes however weaker,
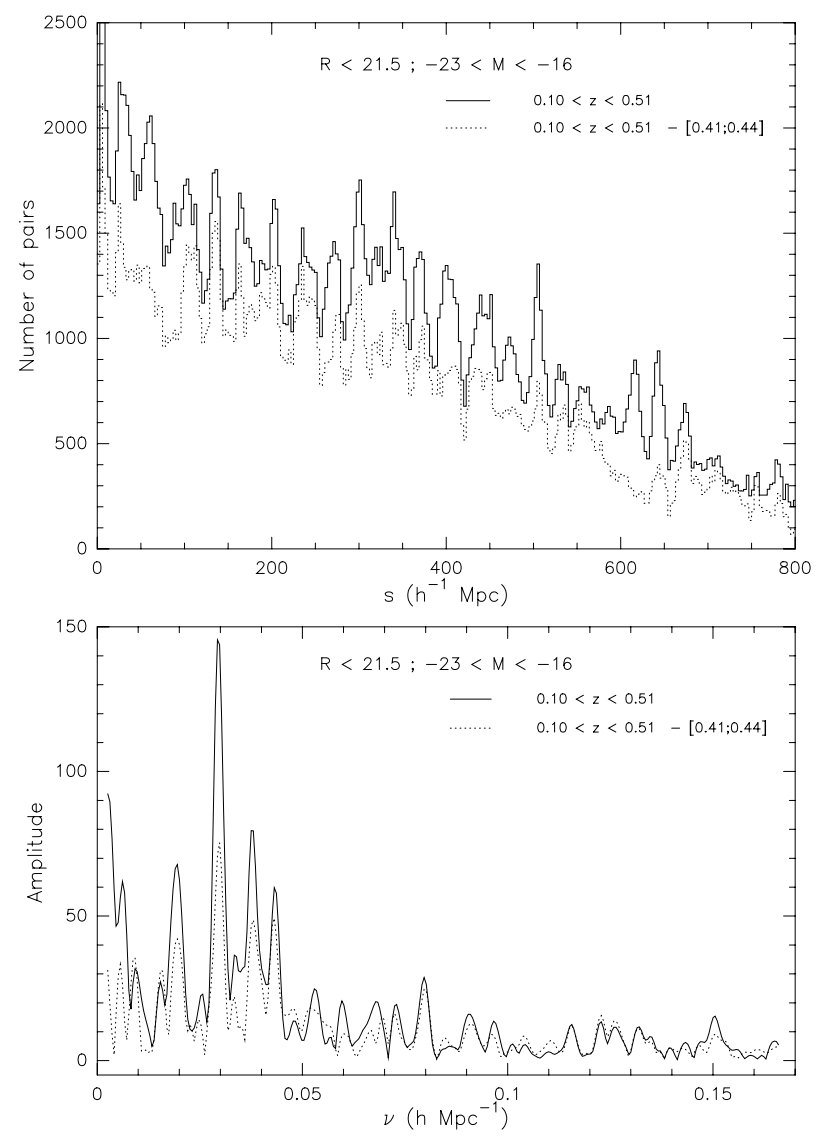

Fig. 6. Pairs separations and the redshift-space correlation function $\xi(s)$ at large scales. The top panel shows the histogram of the data pair separations for all ESO-Sculptor galaxies with $0.10<z<0.51$. The number of pairs decreases as the separation in comoving distance increases, as expected in a pencil-beam survey. Superimposed on the overall trend, numerous peaks are visible and define an apparently regular pattern. The periodogram of the detrended signal is given in the bottom panel. A period of $33.9 h^{-1} \mathrm{Mpc}(v=0.0295)$ is evidenced. Also present are at least four other smaller peaks linked to components with 26.4 and $23.3 \mathrm{~h}^{-1} \mathrm{Mpc}$ periods and the related multiples.

which confirms that the over-dense region also contributes excess pairs in $\xi(s)$ at $25 \lesssim s \lesssim 40 h^{-1} \mathrm{Mpc}$.

We emphasize that the result shown in Fig. 6 is not interpreted as evidence for periodicity in the galaxy distribution (see Yoshida et al. 2001). It is symptomatic of the fact that the ESS does not represent a fair sample of the galaxy distribution, due to its limited volume: as a result, the alternation of voids and walls/filaments along the line-of-sight, and the presence of one over-density parallel to the line-of-sight, both leave an imprint in the correlation function.

\section{4. $\xi(s)$ per galaxy type}

Top panel of Fig. 7 shows $\xi(s)$ for the early-type, late spiral and dwarf galaxies (see Table 1 for details on each sub-sample). For comparison, the power-law fit to $\xi(s)$ for the full sample is also plotted. This comparison shows that the correlation functions for the early-type, fitted by

$s_{0}=7.67 \pm 4.08 h^{-1} \mathrm{Mpc}, \quad \gamma=1.06 \pm 0.18$

(measured for $0.7<s<7.5 h^{-1} \mathrm{Mpc}$ ), has a similar power-law behavior as for the full sample (see Eq. (10) and Fig. 3). The late spiral galaxies present a lower amplitude, and flatter slope of $\xi(s)$ 


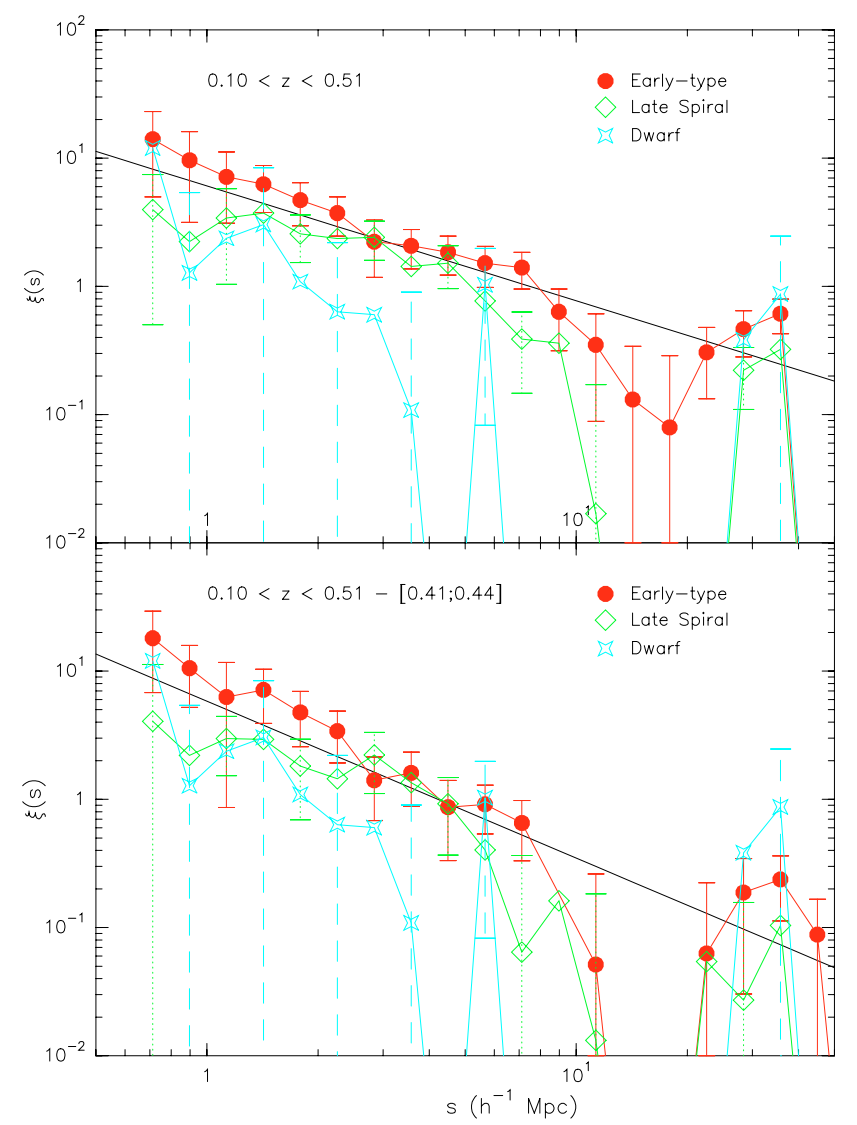

Fig. 7. The redshift-space correlation function $\xi(s)$ for the ESOSculptor sub-samples restricted to the 3 galaxy types: early-type (red circles), late spiral (green diamonds), and dwarf galaxies (cyan open stars). The top panel displays $\xi(s)$ for all samples with $0.10<z<0.51$ while the bottom panel shows the results for the samples in which galaxies within the over-density $0.41<z<0.44$ have been excluded. The same correlation function for the dwarf galaxies (cyan open stars) is shown in both panels as this sample contains no galaxies with $z>0.41$ (see Fig. 2). In each panel, the continuous line indicates the power-law fit to $\xi(s)$ for the full sample (see Fig. 4, and Eqs. (10)-(11)). For clarity, in both panels, the one-sigma error bars are only plotted for every other point of the late spiral and dwarf galaxies.

at small scales than for the early-type galaxies: the power-law fit is defined by

$s_{0}=4.90 \pm 2.55 h^{-1} \mathrm{Mpc}, \quad \gamma=0.96 \pm 0.18$

(measured for $0.7<s<9.0 h^{-1} \mathrm{Mpc}$ ). In contrast, the dwarf galaxies show evidence for a lower clustering amplitude, by a factor $\sim 3.5$, and a steeper slope than for the earlier galaxy types:

$s_{0}=1.94 \pm 0.81 h^{-1} \mathrm{Mpc}, \quad \gamma=2.18 \pm 0.71$

(for $0.7<s<4.0 h^{-1} \mathrm{Mpc}$ ). The marked cut-off in $\xi(s)$ around $s=14 h^{-1} \mathrm{Mpc}$ and the excess power at $s \sim 30 h^{-1} \mathrm{Mpc}$, visible for the 3 spectral classes is symptomatic of the geometry and limited volume of the ESS survey, already discussed in the previous sub-section.

We emphasize that the different clustering for the late spiral and dwarf galaxies in Fig. 7 strongly supports the fact that both galaxy types are physically relevant classes for distinguishing the different components of the galaxy density field. Note also that in Fig. 7 and in most of the following graphs, the mentioned clustering differences among the various sub-samples are often significant at the $2 \sigma$ level at most, due to the limited size of the
ESS. We however take them at face value and derive an interpretation in terms of type segregation in the two-point clustering.

The clustering differences among the ESS galaxy types confirm the visual impression from the ESS redshift cone (Fig. 5): the early-type galaxies are concentrated within the densest regions, corresponding to groups of galaxies, whereas the late spiral and dwarf galaxies also populate the sparser regions of the density field. The observed behavior of the $\xi(s)$ for the 3 ESS galaxy types is related to the type-density relation, originally named "morphology-density" relation (Dressler 1980; Postman \& Geller 1984). The elliptical galaxies tend to populate the densest regions of the Universe, namely clusters and groups of galaxies, and thus tend to have a stronger two-point correlation function on scales of a few $h^{-1} \mathrm{Mpc}$, corresponding to the extent of these concentrations. The late-type spiral galaxies are much more weakly clustered and populate the lowest density regions, whereas the early spiral galaxies have an intermediate behavior.

Recently, using the Sloan Digital Sky Survey, Blanton et al. (2005) showed that it is the present star formation rate which is directly related to the local density, and the correlation with the morphology is a consequence of the relationship between the present star formation rate of a galaxy and its morphology. The ESS spectral classification has the advantage to be tightly related to the present star formation rate (Galaz \& de Lapparent 1998), and the observed trends of $\xi(s)$ seen in Fig. 7 are in agreement with the earlier-type galaxies residing in higher density environments than the late spiral galaxies.

The correlation function for the dwarf galaxies points to a more subtle effect, which is detected here for the first time. At the smallest scales, the amplitude is as high as that for the early-type galaxies, and it steadily decreases at larger and larger scales: at $0.9 \leq s \leq 1.3 h^{-1} \mathrm{Mpc}, \xi(s)$ for the dwarf galaxies is comparable to that for the late spiral galaxies, and it becomes a factor of $\sim 2-10$ times lower at $1.3 \leq s \leq 4.0 h^{-1} \mathrm{Mpc}$. This is consistent with the dwarf galaxies populating the dense groups (small scale behavior of $\xi(s)$ ), whereas on scales of $2-4 h^{-1} \mathrm{Mpc}$, they appear much more weakly clustered than both types of giant galaxies (early-type and late spiral).

Bottom panel of Fig. 7 shows $\xi(s)$ for the ESS early-type and late spiral galaxies when excluding all galaxies with $0.41<$ $z<0.44$. For the dwarf galaxies, we plot the same curve as in Fig. 10 as this sample contains only 2 galaxies with $z>0.41$ (see Fig. 2). The power-law fit to $\xi(s)$ for the full sample without the over-density is also plotted (Eq. (11)). A power-law model is still a good fit to both functions. For the early-type class, we measure

$s_{0}=4.79 \pm 1.69 h^{-1} \mathrm{Mpc}, \quad \gamma=1.47 \pm 0.20$

in the interval $0.7<s<7.5 h^{-1} \mathrm{Mpc}$. For the late spiral galaxies, we obtain

$s_{0}=3.81 \pm 2.41 h^{-1} \mathrm{Mpc}, \quad \gamma=0.96 \pm 0.25$

(for $0.7<s<9.0 h^{-1} \mathrm{Mpc}$ ). Therefore, the effect of removing the over-density onto the correlation functions for the early-type and late spiral galaxies is a decrease in amplitude at scales $s \gtrsim$ $3 h^{-1}$ Mpc: a lower amplitude $s_{0}$ for both types, and a steeper slope for the early-type galaxies. The fact that $\xi(s)$ for both the early-type and late spiral galaxies are affected by removing the over-density indicates that these two populations contribute to the excess clustering in this region.

The differences in the spatial correlation function between different galaxy types may actually be larger than those seen in 


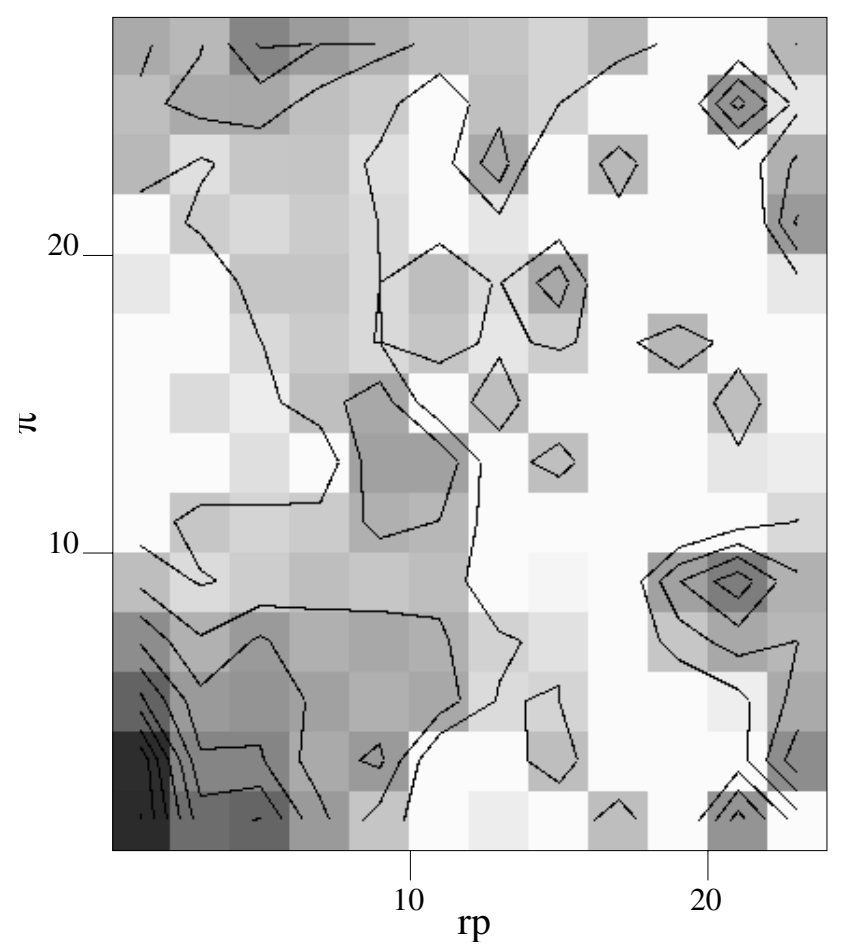

Fig. 8. Line-of-sight/transverse correlation function $\xi\left(r_{\mathrm{p}}, \pi\right)$ for the ESO-Sculptor galaxies with $0.10<z<0.51$, where $r_{\mathrm{p}}$ and $\pi$ are the separations perpendicular and parallel to line-of-sight, both measured in unit of $h^{-1} \mathrm{Mpc}$. The grey levels (from white to black) and the contours of constant $\xi\left(r_{\mathrm{p}}, \pi\right)$ are linearly spaced from $\xi\left(r_{\mathrm{p}}, \pi\right)=0.0$ to $\xi\left(r_{\mathrm{p}}, \pi\right)=3.0$ with steps of $\Delta \xi\left(r_{\mathrm{p}}, \pi\right)=0.25$.

$\xi(s)$, because of the redshift distortions caused by the galaxy peculiar velocities (see Appendix G), which tend to erase the type effects. At small scales $\left(s \sim 1 h^{-1} \mathrm{Mpc}\right)$, the redshift distortions caused by the random motions in dense regions tend to weaken the amplitude increase of $\xi(s)$ for the early-type galaxies with respect to the other types; although there are no rich clusters of galaxies in the ESS, the survey contains contains numerous groups of galaxies (seen as small "fingers-of-god" in Fig. 5) which contribute to this effect at small scales. At larger scales $\left(s \gtrsim 5 h^{-1} \mathrm{Mpc}\right.$ ), the coherent bulk flows tend to increase $\xi(s)$ for the late spiral galaxies, which dominate in the medium and low density environments. In order to free the type measurements from the redshift distortion effect, we calculate in the following sections the projected spatial correlation function $w\left(r_{\mathrm{p}}\right)$.

\section{The projected spatial correlation function $w\left(r_{p}\right)$}

\subsection{General behavior of $w\left(r_{p}\right)$}

To measure $w\left(r_{\mathrm{p}}\right)$, one must first calculate the correlation function $\xi\left(r_{\mathrm{p}}, \pi\right)$ as a function of separation parallel and perpendicular to the line-of-sight (see Appendix G). Figure 8 shows $\xi\left(r_{\mathrm{p}}, \pi\right)$ for all ESS galaxies using the LS estimator (Eq. (B.7)) with the minimum-variance weights (Eq. (D.3)) and bin widths of $2 h^{-1}$ Mpc. The contours of constant clustering amplitude are drawn as solid lines. Figure 8 exhibits a stretching along the lineof-sight ( $\pi$ direction) for separations smaller than $4 h^{-1} \mathrm{Mpc}$, caused by the peculiar velocities within the numerous groups of galaxies present in the ESS (see Fig. 5). The flattening of the contours of $\xi\left(r_{\mathrm{p}}, \pi\right)$ along the line-of-sight due to the coherent infall of galaxies onto the over-dense regions, which was first detected in the 2dFGRS by Hawkins et al. (2003), is hardly seen

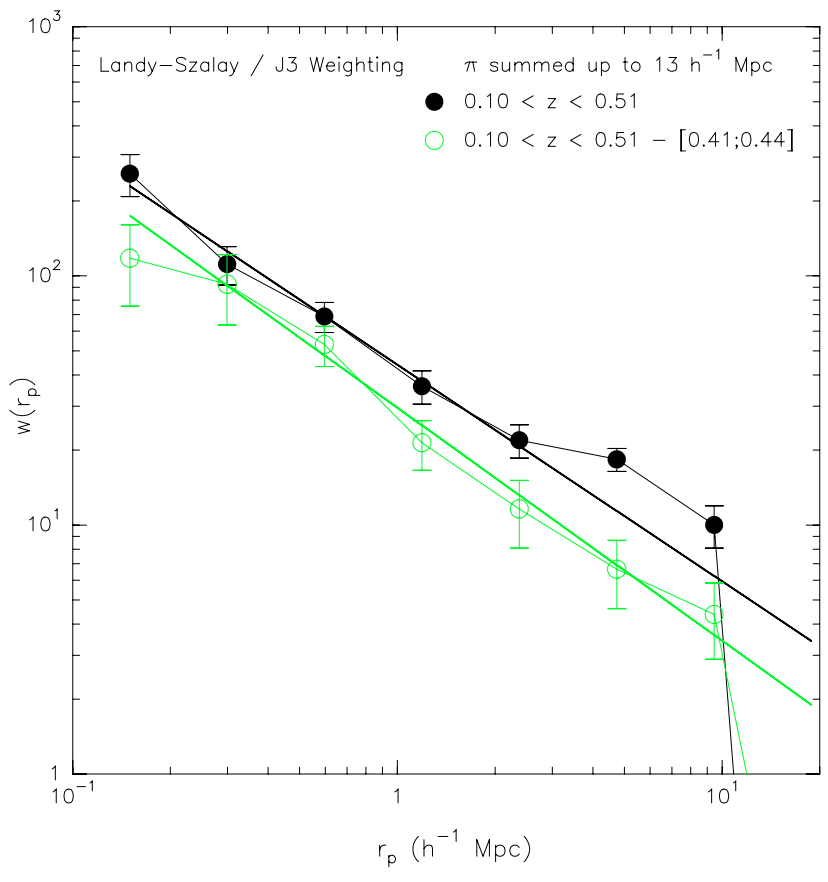

Fig. 9. Projected correlation function $w\left(r_{\mathrm{p}}\right)$ for the ESO-Sculptor subsample including all galaxies (black filled circles), and after removal of the over-density in the interval $0.41<z<0.44$ (green empty circles). The straight lines correspond to the best-fit power-laws whose parameters are given in Eqs. (17) and (18).

here, due to the limited angular extent of the ESS in right ascension, which subtends $\sim 8 h^{-1} \mathrm{Mpc}$ at $z \sim 0.2$.

Because $\xi\left(r_{\mathrm{p}}, \pi\right)$ is a decreasing function of both $r_{\mathrm{p}}$ and $\pi$, one can estimate the projected real-space correlation function $w\left(r_{\mathrm{p}}\right)$ by an integral over $\pi$ (see Eq. (G.2)). In practise, the integral can only be performed to a finite bound, which needs to be determined. To this end, we have calculated the various functions $w\left(r_{\mathrm{p}}\right)$ with integration bounds of $6.31 h^{-1} \mathrm{Mpc}$, $12.59 h^{-1} \mathrm{Mpc}, 25.12 h^{-1} \mathrm{Mpc}$, and $50.12 h^{-1} \mathrm{Mpc}$. The $12.59 h^{-1} \mathrm{Mpc}$ integration bound appears as the smallest bound with evidence for stabilization, and we thus adopt this value. The chosen integration bound of $12.59 \mathrm{~h}^{-1} \mathrm{Mpc}$ also insures that the random noise fluctuations visible at larger values of $\pi$ in Fig. 8 are excluded, as we interpret these as symptomatic of the limited sampling volume of the ESS (see Sect. 4).

The resulting projected real-space correlation function $w\left(r_{\mathrm{p}}\right)$ for the full ESS sample is shown in Fig. 9. In the interval $0.1<r_{\mathrm{p}}<3 h^{-1} \mathrm{Mpc}, w\left(r_{\mathrm{p}}\right)$ is well fitted by a power-law with parameters

$r_{0}=5.25 \pm 1.82 h^{-1} \mathrm{Mpc}, \quad \gamma=1.87 \pm 0.07$,

(the quoted uncertainties in $r_{0}$ and $\gamma$ ignore the correlation between the various points of $w\left(r_{\mathrm{p}}\right)$, and are therefore underestimated).

\subsection{Nature of the over-density at $0.41<z<0.44$}

We also plot in Fig. $9 w\left(r_{\mathrm{p}}\right)$ for the ESS sample without the over-density. The resulting correlation function can be fitted by a power-law over a larger interval of $r_{\mathrm{p}}$ than for the full ESS sample: for $0.2<r_{\mathrm{p}}<10 \mathrm{~h}^{-1} \mathrm{Mpc}$, we obtain the following best fit parameters

$r_{0}=3.50 \pm 1.21 h^{-1} \mathrm{Mpc}, \quad \gamma=1.93 \pm 0.09$ 
The decrease in the amplitude $r_{0}$ of $w\left(r_{\mathrm{p}}\right)$ from Eqs. (17) to (18), with a nearly constant slope, indicates that the over-density at $0.41<z<0.44$ contributes excess pairs of galaxies at all scales. Note that $w\left(r_{\mathrm{p}}\right)$ can be fitted by a power-law over a larger range of scales than $\xi(s)$ (compare Figs. 4 and 9), because the latter function is affected by the peculiar velocities (but see Sect. 6 for discussion of the small deviations at $r_{\mathrm{p}} \simeq 0.15 h^{-1} \mathrm{Mpc}$ and $r_{\mathrm{p}} \simeq 1.0 \mathrm{~h}^{-1}$ Mpc in Fig. 4).

Close examination of Fig. 9 indicates that the over-density in the $0.41<z<0.44$ redshift interval also has a differential effect on $w\left(r_{\mathrm{p}}\right)$ at both small and large scales, with an excess clustering at $r_{\mathrm{p}} \lesssim 0.2 h^{-1} \mathrm{Mpc}$ and $3 \lesssim r_{\mathrm{p}} \lesssim 10 h^{-1} \mathrm{Mpc}$ compared to the power-law fits. The small scale $0.2 \mathrm{~h}^{-1} \mathrm{Mpc}$ corresponds to the typical virial radius of galaxy groups (Yang et al. 2005d), suggesting that the over-density may be due to richer groups than in the rest of the survey. Besides, the $3 h^{-1} \mathrm{Mpc}$ intermediate scale is close to the minimum separation between the galaxy groups (Yang et al. 2005c), which suggest that the groups located within the over-density are more densely clustered than in the rest of the ESS.

Calculation of $w\left(r_{\mathrm{p}}\right)$ therefore clarifies the nature of the overdensity, and quantifies the visual impression that this redshift interval contains richer groups and with a higher spatial contrast than in the rest of the survey (see Fig. 5). This region is thus clearly peculiar. Because of its large size and strong excess in clustering, it has a visible impact on the ESS clustering measurements, which can be interpreted in terms of cosmic variance. When this structure is excluded, the ESS clustering measurements are in good agreement with the other measurements from larger redshift surveys at $z \sim 0$ and $z \sim 0.5$ (see Sect. 7). Moreover, there is enough clustering signal in the ESS survey outside the over-density for allowing us to measure the galaxy correlation functions. Thanks to a detailed account of the various selection effects related to the galaxy types and the luminosity functions, the signal-to-noise in the ESS correlation measurements when excluding the over-density is only slightly reduced. The gain in revealing the 1-halo and 2-halo components of dark matter halos (see Sect. 6) largely compensates for this slight loss in signal-to-noise.

In the following, we thus only consider the correlation functions obtained when the over-density at $0.41<z<0.44$ is excluded, as these best reflect the typical galaxy clustering.

\section{3. $w\left(r_{p}\right)$ per galaxy type}

Figure 10 shows the projected real-space correlation function $w\left(r_{\mathrm{p}}\right)$ for each of the 3 ESS galaxy types: early-type, late spiral and dwarf galaxies. The relative behavior of $w\left(r_{\mathrm{p}}\right)$ for the late spiral galaxies and early-type galaxies is somewhat similar to that seen in $\xi(s)$. The late spiral have a significantly weaker correlation function than for the early-type galaxies at small scales $\left(r_{\mathrm{p}} \leq 0.3 h^{-1} \mathrm{Mpc}\right)$. Then at larger scale, $w\left(r_{\mathrm{p}}\right)$ for the late spiral galaxies has a very similar behavior to that for the early-type galaxies, with a comparable slope, and a factor $\sim 1.5-2.0$ lower amplitude.

A remarkable result in Fig. 10 is that for $r_{\mathrm{p}}>0.3 h^{-1} \mathrm{Mpc}$, the correlation function for the dwarf galaxies is consistent with null clustering within the error bars. This is best seen in Fig. 11, which shows $w\left(r_{\mathrm{p}}\right)$ in linear scale for the dwarf galaxies (with the same color coding as in Fig. 10). Significant clustering of the dwarf galaxies is only detected for $r_{\mathrm{p}} \leq 0.3 h^{-1} \mathrm{Mpc}$ (with a $2 \sigma$ significance level).

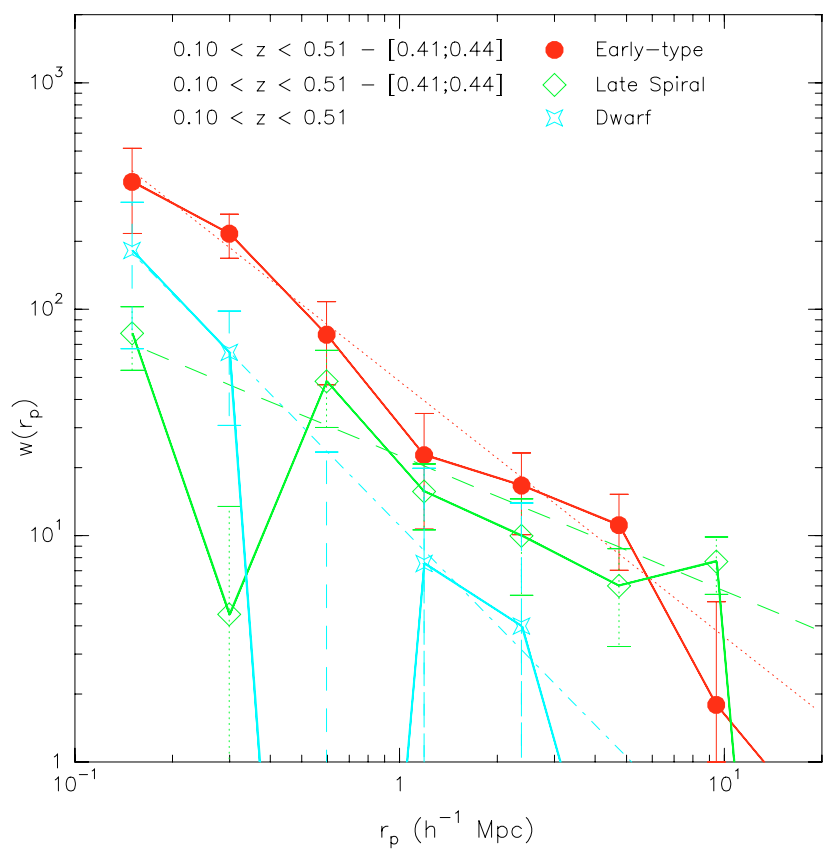

Fig. 10. Projected correlation function $w\left(r_{\mathrm{p}}\right)$ for the ESO-Sculptor subsamples per galaxy type: early-type galaxies (red filled circles), late spiral galaxies (green open diamonds), and dwarf galaxies (cyan open stars). For each galaxy type, the straight line corresponds to the best-fit power-law whose parameters are given in Eqs. (19) to (21). The overdensity in the interval $0.41<z<0.44$ has been removed from the early-type and late spiral samples.

We emphasize the noteworthy differences in the correlation functions for the three galaxy types in Fig. 10. First, pairs of early-type galaxies tend to dominate over pairs of both other galaxy types at all scales (except maybe at $r_{\mathrm{p}} \geq 10 h^{-1} \mathrm{Mpc}$ ), and the effect is even stronger at small scales, $r_{\mathrm{p}} \leq 0.3 \mathrm{~h}^{-1} \mathrm{Mpc}$. Once the early-type galaxies are set aside, the complementary clustering of the late spiral and dwarf galaxies is worth attention: dwarf galaxies have a dominating clustering at $r_{\mathrm{p}} \leq 0.3 \mathrm{~h}^{-1} \mathrm{Mpc}$, and fall-off to null clustering at larger scales; in contrast, Fig. 11 shows that $w\left(r_{\mathrm{p}}\right)$ for the late spiral galaxies have moderate clustering at $r_{\mathrm{p}} \simeq 0.15 h^{-1} \mathrm{Mpc}$, a factor 2 below the dwarf galaxies; then $w\left(r_{\mathrm{p}}\right)$ is consistent with null clustering at $r_{\mathrm{p}} \simeq 0.3 h^{-1} \mathrm{Mpc}$, whereas significant signal is detected at larger scales, from $r_{\mathrm{p}} \simeq$ $0.6 h^{-1} \mathrm{Mpc}$ to $r_{\mathrm{p}} \simeq 10 h^{-1} \mathrm{Mpc}$.

These differences with galaxy types are quantified by the power-law fits of $w\left(r_{\mathrm{p}}\right)$. For the early-type galaxies, we obtain

$r_{0}=3.80 \pm 0.67 h^{-1} \mathrm{Mpc}, \quad \gamma=2.11 \pm 0.10$

(fit over the $0.15 \leq r_{\mathrm{p}} \leq 5 \mathrm{~h}^{-1} \mathrm{Mpc}$ interval). For the late spiral galaxies, both the amplitude and the slope are smaller:

$r_{0}=2.72 \pm 0.64 h^{-1} \mathrm{Mpc}, \quad \gamma=1.60 \pm 0.08$

(fit over the $0.15 \leq r_{\mathrm{p}} \leq 10 \mathrm{~h}^{-1} \mathrm{Mpc}$ interval). In contrast, the dwarf galaxies have an even smaller amplitude and significantly steeper slope than for the giant galaxies:

$r_{0}=1.85 \pm 0.83 h^{-1} \mathrm{Mpc}, \quad \gamma=2.46 \pm 0.38$

(fit over the $0.15 \leq r_{\mathrm{p}} \leq 2.5 h^{-1} \mathrm{Mpc}$ interval).

Binggeli et al. (1990) showed from a local wide-angle survey of low surface brightness galaxies that although dwarf galaxies delineate the same large-scale structures as the giant galaxies, there is a strong segregation among dwarf galaxies: (1) dE lie preferentially in concentrations of galaxies, 


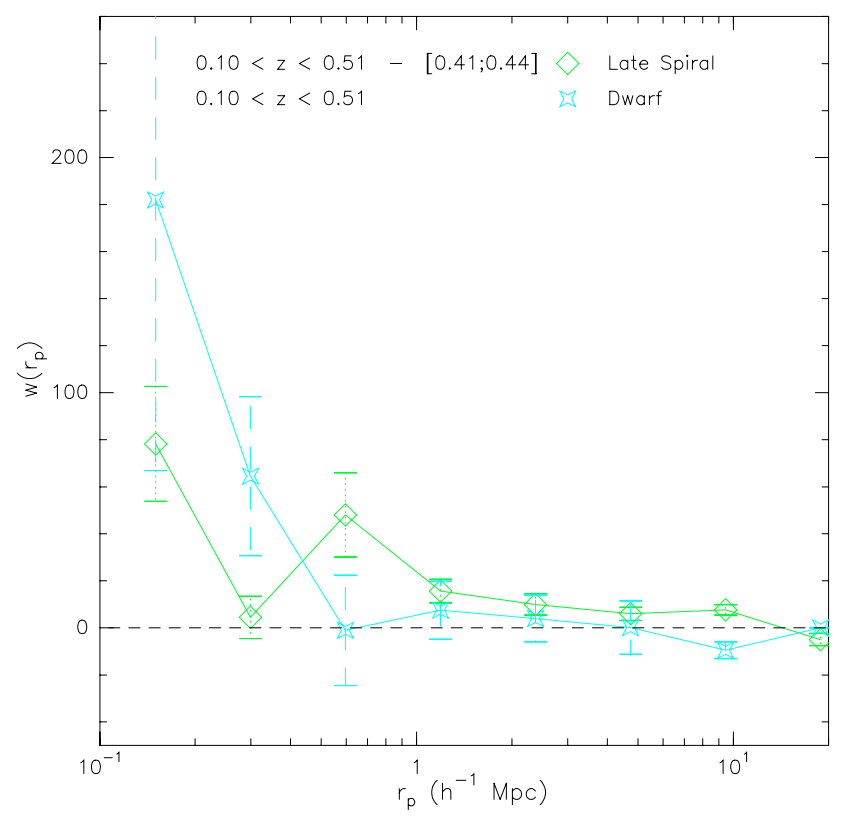

Fig. 11. Projected correlation function $w\left(r_{\mathrm{p}}\right)$ in linear scale for the ESOSculptor late spiral galaxies (green open diamonds) and dwarf galaxies (cyan open stars). The over-density in the interval $0.41<z<0.44$ has been removed from the late spiral sample.

whereas dI are more dispersed; (2) outside clusters, dE also tend to be satellites of giant galaxies. Indeed, studies of dense galaxy clusters show extensive populations of red dwarf or dE galaxies (Andreon \& Cuillandre 2002; Trentham 1997). In less dense environments, one well-studied example being the Local Group, dE galaxies and the even fainter dwarf spheroidal (dSph) galaxies are preferentially found as satellites of the giant spiral galaxies, with typical distances $\leq 0.1 h^{-1} \mathrm{Mpc}$; in contrast, the dI galaxies are more sparsely distributed, and in the Local Group, most of them populate the outskirts at distances of $\leq 0.5 h^{-1} \mathrm{Mpc}$ (http://www.astro.washington.edu/ mayer/LG/LG.html). It is remarkable that the 2 quoted scales are consistent with the interval over which the ESS dwarf galaxies show significant clustering. In the picture of the local Universe, the $\mathrm{dE}$ would be responsible for the dwarf clustering at $r_{\mathrm{p}} \simeq 0.15 h^{-1} \mathrm{Mpc}$ whereas the dI would contribute to the signal at $r_{\mathrm{p}} \simeq 0.3 h^{-1} \mathrm{Mpc}$.

\subsection{Cross-correlation of the giant and dwarf galaxies}

To directly measure how dwarf galaxies cluster around giant galaxies, we plot in Fig. 12 the cross-correlation $w\left(r_{\mathrm{p}}\right)$ between the dwarf galaxies and either the early-type galaxies or the late spiral galaxies. As for the auto-correlation function, we adopt the LS estimator with $J 3$ weighting (see Appendix B). Strikingly, the cross-correlation functions of the dwarf galaxies versus both types of giant galaxies show significant signal, with an amplitude comparable to or intermediate between the autocorrelation functions of the early-type and late spiral galaxies at $r_{\mathrm{p}} \leq 5 \mathrm{~h}^{-1} \mathrm{Mpc}$ (also shown in the graph).

First, we have also calculated the dwarf versus giant galaxy cross-correlation functions after excluding the $\mathrm{dE}$ galaxies: the resulting curves are identical to those shown in Fig. 12, indicating that both the $\mathrm{dE}$ galaxies and the $\mathrm{dI}$ galaxies contribute similarly to the cross-correlation signal. The smaller number of $\mathrm{dE}$ galaxies in the dwarf sub-sample may nevertheless indicate a weaker correlation for these objects, compared to the dI galaxies.

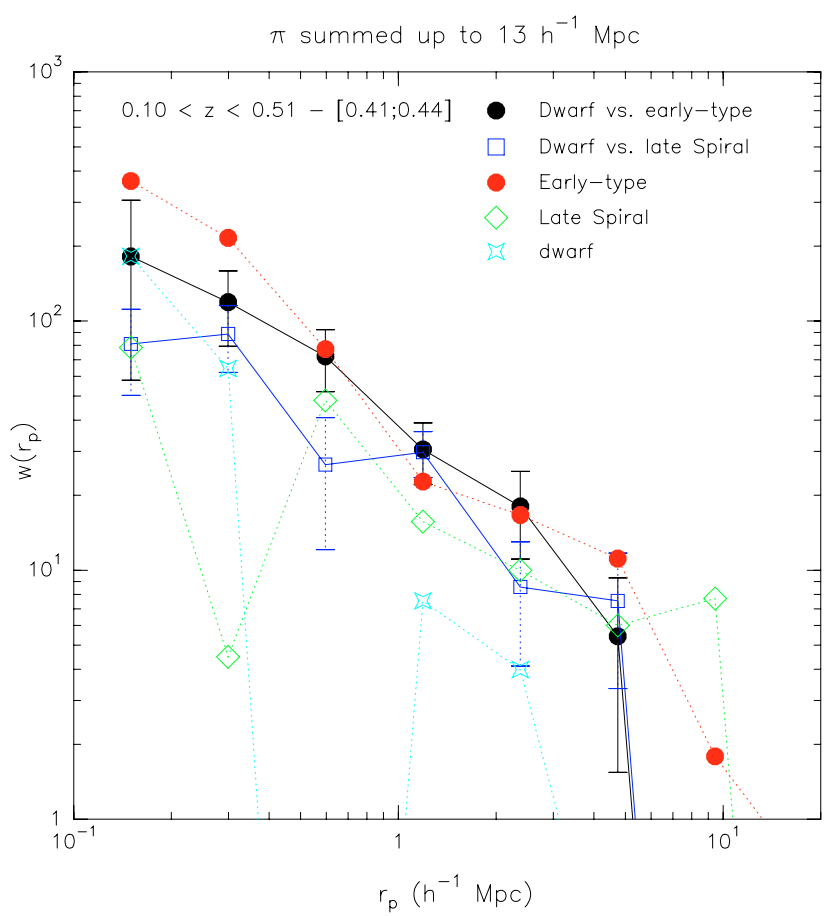

Fig. 12. Projected cross-correlation function $w\left(r_{\mathrm{p}}\right)$ of the ESO-Sculptor dwarf galaxies with the early-type galaxies (black filled circles) and the late spiral galaxies (blue open squares); both curves are connected by solid lines. For comparison, the auto-correlation function for the earlytype (red filled circles), late spiral (green open diamonds), and dwarf galaxies (cyan open stars) are over-plotted, connected by dotted lines. The over-density in the interval $0.41<z<0.44$ has been removed from the early-type and late spiral samples.

In Fig. 12, the cross-correlation of the dwarf versus earlytype galaxies has a $\sim 1.5-2$ higher amplitude ( $1 \sigma$ effect) than that for the dwarf versus late spiral galaxies, over the full scale range $r_{\mathrm{p}} \leq 5 \mathrm{~h}^{-1} \mathrm{Mpc}$. Moreover, both functions have a comparable amplitude as the auto correlation of the dwarf galaxies at small scales $\left(r_{\mathrm{p}} \leq 0.3 h^{-1} \mathrm{Mpc}\right)$. At these scales, the auto-correlation of the early-type galaxies is stronger by a factor $\sim 2(1 \sigma$ effect $)$ than the dwarf/early-type cross-correlation, and by a factor $\sim 3-4$ ( $2 \sigma$ effect) than the dwarf/late spiral cross-correlation. This indicates that at $r_{\mathrm{p}} \leq 0.3 \mathrm{~h}^{-1} \mathrm{Mpc}$, pairs of early-type galaxies may dominate over all the other types of pairs, namely latespiral/late-spiral, dwarf/dwarf, dwarf/early-type, and dwarf/latespiral pairs. It also suggests that the small-scale clustering of the dwarf galaxies may be due to the combined effects of them being satellites of early-type galaxies, and of the early-type galaxy clustering.

In the intermediate scale range $0.6<r_{\mathrm{p}} \leq 5 h^{-1} \mathrm{Mpc}$, mixed pairs of dwarf and giant galaxies appear to contribute equally to the clustering as pairs of giant galaxies, whereas pairs of dwarf galaxies are significantly less frequent. At larger scale $\left(r_{\mathrm{p}}>5 h^{-1} \mathrm{Mpc}\right)$, the cross-correlation signal of dwarf versus giant galaxies vanishes, indicating that the remaining clustering signal is fully dominated by pairs of giant galaxies.

The significant cross-correlation signal between the dwarf galaxies and the late spiral galaxies in the full scale range $r_{\mathrm{p}} \leq$ $5 h^{-1} \mathrm{Mpc}$, seen in Fig. 12, provides direct indication that the $\mathrm{dE}$ and dI galaxies are also clustered in the vicinity of the late spiral galaxies. However, the different behavior of the auto-correlation functions for the two galaxy populations, with the dominating clustering of the dwarf galaxies at $r_{\mathrm{p}} \leq 0.3 h^{-1} \mathrm{Mpc}$ and that 


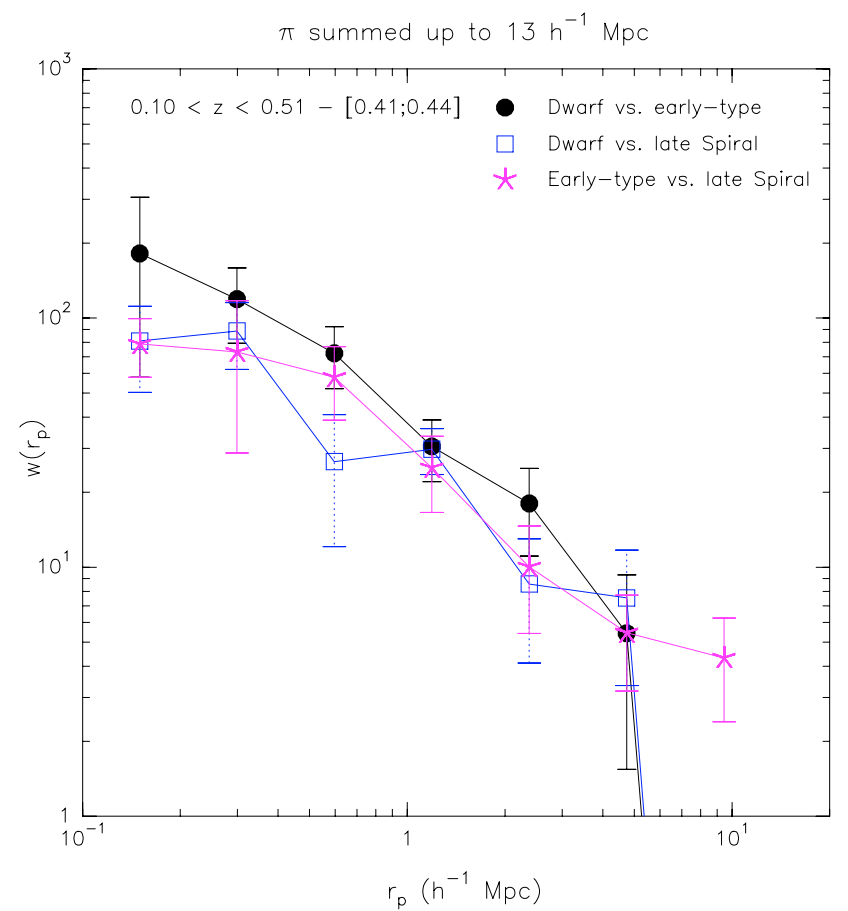

Fig. 13. The same cross-correlation functions as in Fig. 12, namely the ESO-Sculptor dwarf versus early-type galaxies (filled black circles) and the dwarf versus late spiral galaxies (blue open squares), overlaid with the cross-correlation function of the late spiral versus early-type galaxies (magenta asterisks). The over-density in the interval $0.41<z<0.44$ has been removed from the early-type and late spiral samples.

of the late spiral galaxies at larger scales, may indicates that the two populations have distinct distributions at these scales.

In Fig. 13, we compare the cross-correlation function of the dwarf versus late spiral galaxies with the cross-correlation of the late spiral versus early-type galaxies. We observe that at all scales with $r_{\mathrm{p}} \leq 5 \mathrm{~h}^{-1} \mathrm{Mpc}$, both functions are indistinguishable $^{1}$, whereas the amplitude of the dwarf versus early-type cross-correlation function (also plotted in Fig. 13) is a factor of 1.5-2 higher ( $1 \sigma$ effect). This suggests the interesting property that the clustering of the dwarf galaxies around late spiral galaxies might be a consequence of how both galaxy types cluster in the environment of early-type galaxies. This is further developed in the next sub-section, where we interpret these observed clustering properties in terms of the occupation of the dark matter halos by the different galaxy types.

\section{Interpretation in terms of dark matter haloes}

Based on respectively the $2 \mathrm{dF}$ Galaxy Redshift Survey (2dFGRS hereafter) and the Sloan Digital Sky Survey (SDSS hereafter), Magliocchetti \& Porciani (2003) and Zehavi et al. (2004) showed evidence for a deviation of the projected correlation function $w\left(r_{\mathrm{p}}\right)$ from a power-law, with a change of slope at $r_{\mathrm{p}} \simeq 2 \mathrm{~h}^{-1} \mathrm{Mpc}$. Both groups of authors interpret this inflexion point as the transition from the small-scale regime where pairs of galaxies located within the same dark matter halos dominate (denoted hereafter "1-halo component"), to the large-scale regime where pairs of galaxies residing in separate halos overtake the

1 The late spiral versus early-type cross-correlation function might be smoother than the dwarf versus late spiral cross-correlation because there are $72 \%$ more early-type galaxies than dwarf galaxies in the considered samples (see Table 1). clustering signal (denoted hereafter "2-halo component"), this transition occurring near the virial diameter of the halos. This interpretation is further confirmed by the excellent fit of the observed deviations of $w\left(r_{\mathrm{p}}\right)$ from a power-law using the general formalism of the "halo occupation distribution" (HOD hereafter; Magliocchetti \& Porciani 2003; Zehavi et al. 2004, 2005). This approach has the advantage of providing an analytical description of the clustering of biased galaxy populations (Benson et al. 2000; Berlind \& Weinberg 2002), and comparison with observations provides constraints on the HOD parameters.

The projected correlation function $w\left(r_{\mathrm{p}}\right)$ for the full ESS sample without the over-density at $0.41<z<0.44$, shown in Fig. 9, displays a similar deviation from a power-law, with an inflexion point at $\sim 1 h^{-1} \mathrm{Mpc}$. At scales smaller than the inflexion point, the correlation function $w\left(r_{\mathrm{p}}\right)$ is poorly fitted by a power-law. This matches the theoretical expectation that the 1-halo component follows the halo mass function, which flattens off at small scales (Zehavi et al. 2004; Jenkins et al. 2001). At $r_{\mathrm{p}} \sim 1 \mathrm{~h}^{-1} \mathrm{Mpc}$, the 2-halo regime takes over and is determined by the matter correlation function and the halo bias (Zehavi et al. 2004). At larger scales, the correlation function is also expected to deviate from a power-law, but this is not visible in Fig. 9, due to limited statistics.

\subsection{Dependence on galaxy type}

We now turn to the analysis of the ESS correlation functions by galaxy type, as they provide a new insight into the contribution of the different galaxy populations to the halo components. Figure 10 shows that for the three ESS galaxy types, $w\left(r_{\mathrm{p}}\right)$ does deviate from a simple power-law fit. For the early-type galaxies, the 1-halo to 2-halo transition is clearly detected, and is located at $r_{\mathrm{p}} \simeq 1 \mathrm{~h}^{-1} \mathrm{Mpc}$. Moreover, both the 1-halo and 2-halo components have the similar non power-law behavior as that measured from the 2dFGRS early-type galaxies by Magliocchetti \& Porciani (2003); the two components are modelled by a standard mass profile (Navarro et al. 1997), and a prescription for the two-point correlation function of dark matter halos respectively. Similar results and modelling are derived from the SDSS by Zehavi et al. (2005).

Nevertheless, there are indications of differences between the ESS early-type clustering and those measured locally from the 2dFGRS and SDSS: an apparently smaller transition scale of $r_{\mathrm{p}} \simeq 1 h^{-1} \mathrm{Mpc}$, instead of $r_{\mathrm{p}} \simeq 2 h^{-1} \mathrm{Mpc}$; and the fall-off of the ESS large-scale clustering power at $r_{\mathrm{p}} \geq 10 \mathrm{~h}^{-1} \mathrm{Mpc}$, whereas both the $2 \mathrm{dFGRS}$ and SDSS have significant clustering out to at least $r_{\mathrm{p}} \simeq 20 h^{-1} \mathrm{Mpc}$. The cut-off at $r_{\mathrm{p}} \simeq 10 h^{-1} \mathrm{Mpc}$ for all the ESS auto and cross-correlation functions is likely due to the limited angular extent of the survey: its angular size is $\sim 1.0^{\circ}$, which subtends $r_{\mathrm{p}} \simeq 10 \mathrm{~h}^{-1} \mathrm{Mpc}$ at the median redshift of the survey $(z=0.3)$; as a result, any existing correlation signal beyond $\sim 10 h^{-1} \mathrm{Mpc}$ cannot be detected in $w\left(r_{\mathrm{p}}\right)$. In contrast, the smaller transition scale in the ESS may be real and could be due to evolution effects related to the higher redshift range of the ESS $(0.1 \leq z \leq 0.5)$ compared to $z \leq 0.1$ for the 2 dFGRS and SDSS.

Figure 10 shows that the higher amplitude and slope of the ESS early-type auto-correlation compared to that for the late spiral galaxies can be decomposed into a $50 \%$ higher amplitude but similar slope for the 2-halo component, and a factor $\sim 2-4$ higher amplitude and significantly flatter slope for the 1-halo component. This is in good agreement with the results obtained by Magliocchetti \& Porciani (2003) and Zehavi et al. (2005) from 
the 2dFGRS and SDSS respectively. These authors successfully model the clustering of both galaxy types (early/red, late/blue) using the HOD prescription, which confirms that both galaxy types follow the dark matter distribution within the halos.

One of the major predictions of hierarchical clustering is that the most massive halos have the strongest two-point clustering (Zheng et al. 2002). In this context, the $50 \%$ excess amplitude of the early-type auto-correlation function over that for the late spiral galaxies in the 2-halo regime indicates that early-type galaxies tend to reside in more massive halos than the late spiral galaxies. Moreover, the relative behavior in the 1-halo regime shows that the excess of early-type clustering is even stronger within the halos, and increases at higher clustering levels. Given that the clustering is stronger in higher density regions, at all scales from 0.1 to $30 h^{-1} \mathrm{Mpc}$ (Abbas \& Sheth 2006), and that the density increases towards the center of the halos (Navarro et al. 1997), the excess small scale clustering of the ESS early-type galaxies is consistent with them being preferentially located at smaller radii from the halos centers than the late spiral galaxies.

The auto-correlation function of the ESS dwarf galaxies can also be conveniently interpreted in terms of halo membership. Figure 10 shows a clear 1-halo component which falls off at $r_{\mathrm{p}}>0.3 h^{-1} \mathrm{Mpc}$, thus indicating that the dwarf galaxies are confined to the densest parts of the halos. This is in agreement with the fact that these regions are dominated by early-type galaxies, and that dwarf galaxies are preferentially satellites of early-type galaxies (see Sect. 5.4). A possible weak 2-halo component of the dwarf galaxies at $1 \leq r_{\mathrm{p}} \leq 2 h^{-1} \mathrm{Mpc}$ (Fig. 10) could be the replica of the early-type 2-halo component with the appropriately scaled amplitude.

Information on the degree of mixing of the early-type and late spiral galaxies within halos can be obtained from their crosscorrelation function. It is displayed in Fig. 14, where it is compared with the auto-correlation functions $w\left(r_{\mathrm{p}}\right)$ for both galaxy types. The cross-correlation is close to the mean of the two auto-correlation functions at all scales but $r_{\mathrm{p}} \sim 0.15 \mathrm{~h}^{-1} \mathrm{Mpc}$. In the 2-halo regime, this is naturally expected and provides no additional information over the auto-correlation functions (Zehavi et al. 2005). In contrast, in the 1-halo regime, it suggests that both galaxy types are well mixed within halos at $r_{\mathrm{p}} \geq 0.3 \mathrm{~h}^{-1} \mathrm{Mpc}$, that is that they do not avoid residing in the same halos. Using the cross-correlation function, Zehavi et al. (2005) also found a good level of mixing of the red and blue SDSS galaxies, at all scales of the 1-halo regime.

Nevertheless, in the ESS analysis presented here, the identical amplitude of the early-type versus late spiral crosscorrelation function and the late spiral auto-correlation function at $r_{\mathrm{p}} \simeq 0.15 h^{-1} \mathrm{Mpc}$, together with a factor $\sim 5$ higher amplitude for the early-type auto-correlation function at this scale indicate a lack of mixing of the two galaxy types: pairs of earlytype galaxies dominate over pairs of late spiral galaxies and cross-pairs of early-type and late spiral galaxies. We notice that $r_{\mathrm{p}} \simeq 0.15 \mathrm{~h}^{-1} \mathrm{Mpc}$ is also the smallest detected scale of correlation signal in the 2dFGRS (Magliocchetti \& Porciani 2003) and SDSS (Zehavi et al. 2005), which have higher statistics than the ESS. This scale is likely to correspond to the highest density regions, hence to the very centers of the most massive halos (Navarro et al. 1997). The cross-correlation functions therefore bring the additional information that the centers of the most massive halos are dominated by pairs of early-type galaxies. Conversely, these observations suggest that the late spiral galaxies tend to lie either in the outer regions of the densest halos or in the centers of less dense halos.

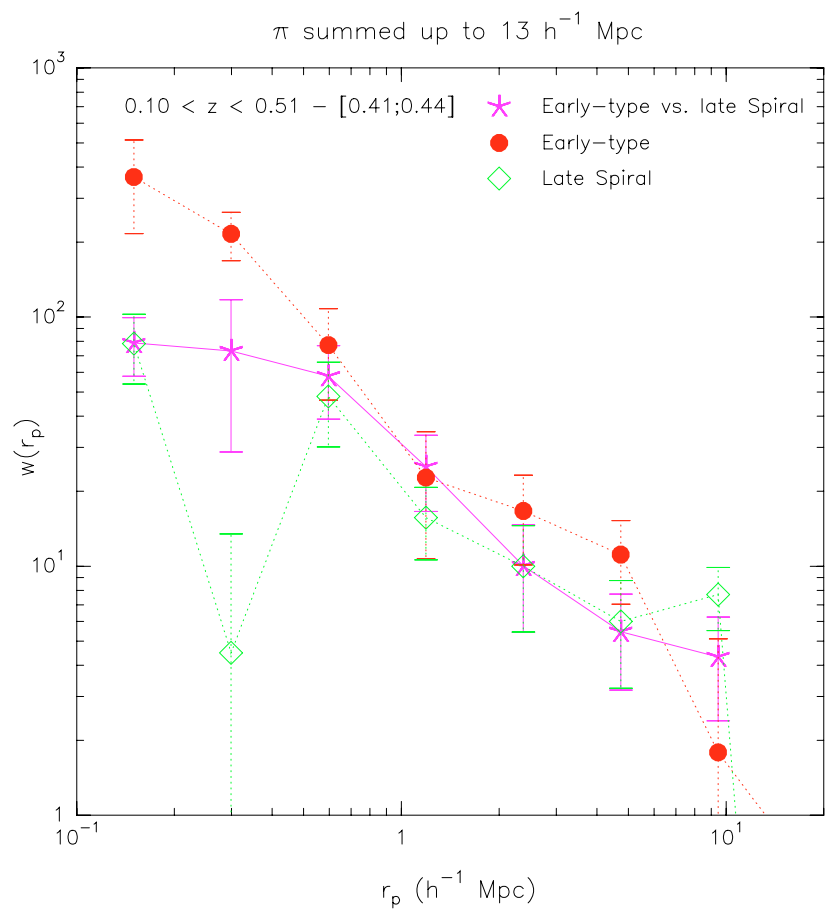

Fig. 14. Projected cross-correlation function $w\left(r_{\mathrm{p}}\right)$ of the ESO-sculptor late spiral galaxies with the early-type galaxies (magenta asterisks). For comparison, the auto-correlation functions for the early-type galaxies (red filled circles) and the late spiral galaxies (green open diamonds) are also shown. The over-density in the interval $0.41<z<0.44$ has been removed from the early-type and late spiral samples.

These results are consistent with the HOD-based model proposed by Zehavi et al. (2005), in which blue galaxies are the central galaxies of the least massive halos, whereas red-type galaxies are the central galaxies in all other halos, including the most massive. Such a segregation effect is also detected by Magliocchetti \& Porciani (2003), whose HOD modelling using a common dark matter profile can successfully predict $w\left(r_{\mathrm{p}}\right)$ for both the early-type and late-type galaxies, provided that the former populate the halos out to one virial radius, and the latter are allowed to extend out to twice that distance. The marked deficit of pairs of ESS late spiral galaxies at $r_{\mathrm{p}} \simeq 0.3 h^{-1} \mathrm{Mpc}$ (see Fig. 10) could be interpreted as further evidence that a significant part of these objects tend to populate the outer regions of the halos.

\subsection{Dependence on galaxy luminosity}

It has been widely observed that intrinsically luminous galaxies cluster more strongly than faint ones (e.g. Benoist et al. 1996; Guzzo et al. 2000; Zehavi et al. 2002). To examine whether such systematic variations are present in the ESS, we separate the early-type and late spiral galaxies into bright and faint subsamples using the median absolute magnitude of each sample: -21.14 for the early-type galaxies, -20.56 for the late spiral galaxies; the corresponding numbers of galaxies for the subsamples are listed in Table 1. The resulting projected autocorrelation functions $w\left(r_{\mathrm{p}}\right)$ for both the early-type and late spiral sub-samples are displayed in Fig. 15, using filled, open symbols for the bright and faint sub-samples respectively.

For the early-type galaxies (left panel of Fig. 15), the correlation function is unchanged when restricting to the bright subsample, except at $r_{\mathrm{p}} \simeq 0.15 \mathrm{~h}^{-1} \mathrm{Mpc}$ where the signal decreases 

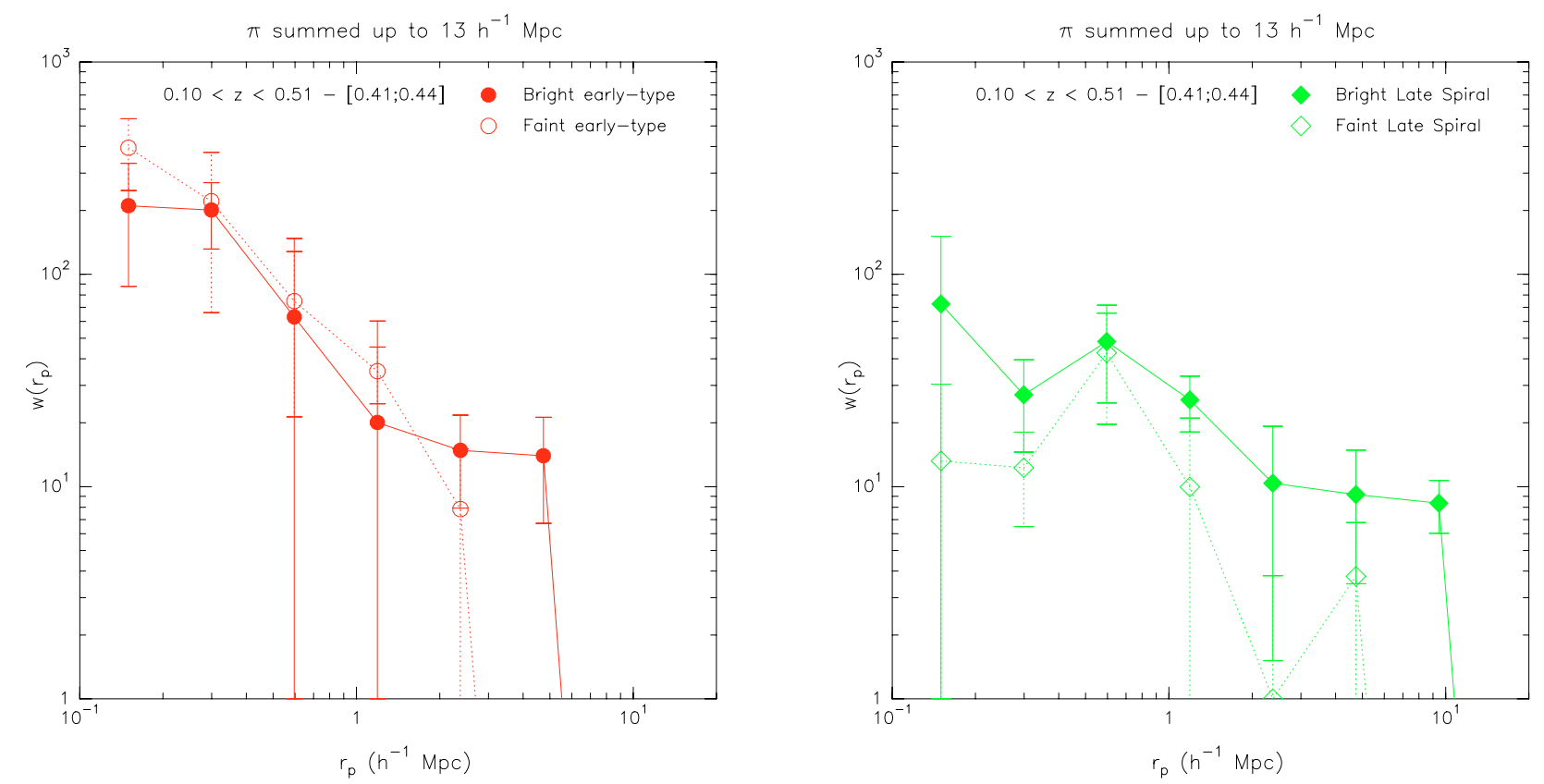

Fig. 15. Projected correlation function $w\left(r_{\mathrm{p}}\right)$ for the ESO-sculptor sub-samples split at their median absolute magnitude: the early-type, late spiral galaxies are shown in the left and right panels respectively; the filled symbols and solid lines mark the bright sub-samples, the open symbols and dotted lines the faint sub-samples. In both panels, the over-density in the interval $0.41<z<0.44$ has been removed from the samples.

by a factor $\sim 2$, and at $r_{\mathrm{p}} \simeq 10 h^{-1} \mathrm{Mpc}$ where the signal vanishes. The faint sub-sample has nearly the same clustering strength as the full early-type sample at $r_{\mathrm{p}} \lesssim 1 h^{-1} \mathrm{Mpc}$; then the correlation function decreases to nearly half that for the bright galaxies at $r_{\mathrm{p}} \simeq 2.5 \mathrm{~h}^{-1} \mathrm{Mpc}$, and the power vanishes beyond. Altogether, the relative behavior of the early-type luminosity sub-samples indicates that in the 1-halo regime, both sub-samples contribute equally, with maybe a dominant contribution from the fainter galaxies at the very small scale $r_{\mathrm{p}} \simeq 0.15 \mathrm{~h}^{-1} \mathrm{Mpc}$. Whereas in the 2-halo regime, the bright early-type galaxies tend to dominate at $r_{\mathrm{p}} \geq 2.5 h^{-1} \mathrm{Mpc}$. This is in agreement with the results of Zehavi et al. (2005) that bright red galaxies exhibit the strongest clustering at large scale, whereas faint red galaxies exhibit the strongest clustering at small scales. The authors reproduce this behavior using an HOD model in which nearly all faint red galaxies are satellite in high mass halos.

When compared to the early-type galaxies, the relative clustering of the faint and bright late spiral galaxies (right panel of Fig. 15) shows a somewhat similar behavior at large scales, and a difference at small scales. Indeed, the 2-halo component of the bright late spiral sub-sample is consistent with a factor $\sim 2-5$ ( $1 \sigma$ deviation) stronger clustering than for the faint sub-sample. In contrast to the early-type galaxies, the 1-halo component of the bright late spiral sub-sample tends to have stronger clustering than for the faint sub-sample. This is again in agreement with the steadily decreasing amplitude of $w\left(r_{\mathrm{p}}\right)$ for the faint blue SDSS galaxies. At variance with the early-type galaxies, there is no indication of excess clustering of the faint late spiral galaxies at $r_{\mathrm{p}} \simeq 0.15 \mathrm{~h}^{-1} \mathrm{Mpc}$; on the contrary, they might be less clustered than their bright analogs.

Yet another prediction of hierarchical clustering is that luminous galaxies are expected to be preferentially located within massive halos, which in turn are more strongly clustered. Here again, although the clustering deviations in the luminosity subsamples of the early-type and late spiral galaxies are only significant at the $2 \sigma$ level at most, we take them at face value and derive an interpretation in terms of dark matter halo membership. The excess clustering in the 2-halo regime of the ESS bright subsamples over the faint sub-samples, which is detected for both the early-type and late spiral galaxies in Fig. 15, is consistent with this expected property of hierarchical clustering.

In contrast, the difference in the relative behavior of the 1-halo components for the early-type and late spiral galaxies suggests that the two galaxy types trace the dark matter profiles of the halo in a different way. In the previous section, we suggested that early-type galaxies tend to occupy the centers of the most massive halos, and that late spiral galaxies tend to lie either in the centers of less dense halos and/or in the outer regions of the densest halos. The additional information brought here is that this is nearly independent of luminosity for the early-type galaxies, whereas faint late spiral galaxies might tend to reside in even less dense regions than their bright analogs. This implies a specific spatial segregation of the early-type and late spiral galaxies inside the dark matter halos.

\section{Comparison with other surveys}

Before the establishment of the currently standard cosmology $\Omega_{\mathrm{m}}=0.3$ and $\Omega_{\Lambda}=0.7$ (Riess et al. 1998; Perlmutter et al. 1999; Phillips et al. 2001; Tonry et al. 2003), various surveys have obtained measures of the galaxy two-point correlation function in redshift space and/or projected separation assuming either a low or high matter density Universe and a null cosmological constant (at $z \simeq 0.1-0.5$ : Cole et al. 1994; Le Fevre et al. 1996; Small et al. 1999; Guzzo et al. 2000; Hogg et al. 2000; Carlberg et al. 2000; Shepherd et al. 2001; at $z \simeq 0$ : Loveday et al. 1992; Park et al. 1994; Baugh 1996; Tucker et al. 1997; Ratcliffe et al. 1998b; Giuricin et al. 2001). To compare our results with those from the other surveys, we thus consider only the more recent measurements, which use the new standard cosmological parameters.

We specifically focus on the projected-separation correlation function $w\left(r_{\mathrm{p}}\right)$ and list in Table 3 its amplitude and slope obtained for the full ESS sample without the over-density in 
Table 3. Parameters of power-law fits to the projected-separation correlation function for other redshift surveys.

\begin{tabular}{|c|c|c|c|c|c|c|}
\hline $\begin{array}{l}\text { Survey } \\
\text { Galaxy type }\end{array}$ & Redshift range & Absolute magnitude range & Numb. & $r_{0}\left(h^{-1} \mathrm{Mpc}\right)$ & $\gamma$ & Reference \\
\hline ESS & $0.1 \leq z \leq 0.51 \dagger$ & $M_{R_{\mathrm{c}}}-5 \log h \leq-16.0$ & 654 & $3.50 \pm 1.21$ & $1.93 \pm 0.09$ & present analysis \\
\hline VVDS & $0.1 \leq z \leq 0.5$ & $M_{B}-5 \log h \leq-17.0$ & 1089 & $3.0 \pm 0.5$ & $1.7 \pm 0.1$ & Pollo et al. (2006) \\
\hline SDSS & $z \leq 0.04$ & $M_{r}-5 \log h \leq-18.0 *$ & 8730 & $3.7 \pm 0.3$ & $1.87 \pm 0.05$ & Zehavi et al. (2005) \\
\hline SDSS & $z \leq 0.06$ & $M_{r}-5 \log h \leq-17.0 *$ & 23560 & $4.6 \pm 0.2$ & $1.89 \pm 0.03$ & id. \\
\hline 2dFGRS & $0.01 \leq z \leq 0.20$ & & 165659 & $5.0 \pm 0.3$ & $1.70 \pm 0.03$ & id. \\
\hline \multicolumn{7}{|l|}{ ESS: } \\
\hline - early-type galaxies & $0.1 \leq z \leq 0.51$ & $M_{R_{\mathrm{c}}}-5 \log h \leq-16.0$ & 218 & $3.80 \pm 0.67$ & $2.11 \pm 0.10$ & present analysis \\
\hline - late spiral galaxies & $0.1 \leq z \leq 0.51$ & $M_{R_{\mathrm{c}}}-5 \log h \leq-16.0$ & 279 & $2.72 \pm 0.64$ & $1.60 \pm 0.08$ & id. \\
\hline $\begin{array}{l}\text { - dwarf galaxies } \\
\text { VVDS: }\end{array}$ & $0.1 \leq z \leq 0.51$ & $M_{R_{\mathrm{c}}}-5 \log h \leq-16.0$ & 159 & $1.85 \pm 0.83$ & $2.46 \pm 0.38$ & id. \\
\hline - elliptical/S0 galaxies & $0.2 \leq z \leq 0.6$ & $M_{B_{A B}}-5 \log h \leq-15.0$ & 164 & $3.1 \pm 0.8$ & $2.4 \pm 0.3$ & Meneux et al. (2006) \\
\hline - Sb-Sc galaxies & $0.2 \leq z \leq 0.6$ & $M_{B_{A B}}-5 \log h \leq-15.0$ & 736 & $2.8 \pm 0.4$ & $1.9 \pm 0.2$ & id. \\
\hline $\begin{array}{l}\text { - Magellanic irregulars } \\
\text { 2dFGRS: }\end{array}$ & $0.2 \leq z \leq 0.6$ & $M_{B_{A B}}-5 \log h \leq-15.0$ & 507 & $1.7 \pm 0.3$ & $1.8 \pm 0.1$ & id. \\
\hline - passive galaxies & $0.01 \leq z \leq 0.20$ & & 36318 & $6.05 \pm 0.35$ & $1.94 \pm 0.03$ & Madgwick et al. (2003) \\
\hline \multicolumn{7}{|l|}{ SDSS: } \\
\hline - red galaxies & $0.03 \leq z \leq 0.07$ & $-20 \leq M_{r}-5 \log h \leq-19.0 *$ & 5804 & $5.7 \pm 0.3$ & $2.10 \pm 0.05$ & Zehavi et al. (2005) \\
\hline - blue galaxies & $0.03 \leq z \leq 0.07$ & $-20 \leq M_{r}-5 \log h \leq-19.0 *$ & 8419 & $3.6 \pm 0.3$ & $1.70 \pm 0.05$ & id. \\
\hline \multicolumn{7}{|l|}{ SDSS: } \\
\hline$-\operatorname{red} w(\theta)$ & & $-21 \leq M_{r}-5 \log h$ & 343 & $6.59 \pm 0.17$ & $1.96 \pm 0.05$ & Budavári et al. (2003) \\
\hline - blue $w(\theta)$ & & $-21 \leq M_{r}-5 \log h$ & 316 & $4.51 \pm 0.19$ & $1.68 \pm 0.09$ & id. \\
\hline
\end{tabular}

Notes: $\dagger$ The over-density in the interval $0.41<z<0.44$ is excluded from the listed redshift interval. * The star symbol in the absolute magnitude range column indicates that the corresponding sample is volume limited to the quoted absolute magnitude limits. Elsewhere, the indicated absolute magnitude results from the combination of the apparent magnitude and redshift limits of the sample.

the redshift interval $0.41 \leq z<0.44$. For comparison, we list the measurements from the SDSS, the 2dFGRS, and the VIMOS-VLT Deep Survey (VVDS). The ESS values are in good agreement with those obtained from the VVDS (Pollo et al. 2006) in a similar redshift interval. The comparatively higher ESS amplitude $r_{0}=5.25 \pm 1.82 h^{-1} \mathrm{Mpc}$, derived when the over-density at $0.41 \leq z<0.44$ is included, strengthens our conclusion that this structure is a peculiar region of the survey.

The parameters of $w\left(r_{\mathrm{p}}\right)$ derived from the ESS are also consistent with those obtained from the 8730 SDSS galaxies with $M_{r} \leq-18.0$ (Zehavi et al. 2005). Our value of $r_{0}$ is nevertheless below that obtained from 23560 SDSS galaxies with $M_{r} \leq-17.0$ (Zehavi et al. 2005), and that from 165659 2dFGRS galaxies (for supposedly $M_{b_{J}} \leq-17.5$, Hawkins et al. 2003). In a $\Lambda$ CDM universe undergoing hierarchical clustering, an evolution in $r_{0}$ is expected between redshifts 0 and 0.5 , specifically a decrease by $\sim 0.5 h^{-1} \mathrm{Mpc}$ (Benson et al. 2001; Kauffmann et al. 1999b). Although such a variation is compatible with the comparison of the ESS and the local SDSS and 2dFGRS correlation measurements, the wide error bars of the ESS correlation function do not allow us to draw any firm conclusion on the evolution in $r_{0}$.

The second part of Table 3 lists the parameters of the powerlaw fits to $w\left(r_{\mathrm{p}}\right)$ for the same surveys as quoted in the top of the Table, split by galaxy type. For the VVDS, we list the measurements for the elliptical/SO (type 1), Sb-Sc spiral (type 2) and Magellanic irregular galaxies (type 4) (Meneux et al. 2006); note that we do not consider the correlation function for galaxy types Sc-Sd (type 3) measured by Meneux et al. (2006), because the results are nearly identical to those for types Sb-Sc; moreover, among the ESS late spiral galaxies, intermediate-type galaxies which correspond to Sb-Sc type dominate in number over later type galaxies (see Table 1). Both the ESS early-type and VVDS elliptical/S0 on the one hand, and the ESS late spiral and the VVDS Sb-Sc galaxies on the other hand, have values of the amplitude $r_{0}$ and the slope $\gamma$ which are in $1 \sigma$ agreement. The amplitude for the ESS dwarf galaxies and the VVDS Magellanic irregulars are also in good agreement, whereas the slope is significantly steeper in the ESS. The slope of $w\left(r_{\mathrm{p}}\right)$ for the ESS dwarf galaxies could be even steeper as the signal at $r_{\mathrm{p}} \geq 1 h^{-1} \mathrm{Mpc}$ may not be real in this population (see Fig. 10).

At the smaller redshifts covered by the SDSS and 2dFGRS, the correlation function for the red galaxies has a higher amplitude and steeper slope than for the blue galaxies (Zehavi et al. 2005; Madgwick et al. 2003), indicating similar segregation effects as in the ESS. This type effect was also detected from the SDSS angular correlation function (Budavári et al. 2003), with good agreement in the power-law parameters. Note however that the correlation functions by galaxy type for the SDSS and 2dFGRS have higher amplitudes than for the ESS and VVDS, which may also be the trace of clustering evolution.

\section{Summary of results}

We calculate the two-point correlation function for the ESOSculptor redshift survey. The sample is limited to the 765 galaxies with $R_{\mathrm{c}} \leq 21.5$ in the redshift interval $0.1 \leq z \leq 0.51$. We use on the one hand the template free spectral classification of the sample into early, intermediate, and late-type galaxies, which correspond to the following mixes of morphological type: $\mathrm{E}+\mathrm{SO}+\mathrm{Sa}, \mathrm{Sb}+\mathrm{Sc}$, and $\mathrm{Sc}+\mathrm{Sd} / \mathrm{Sm}$, respectively (Galaz \& de Lapparent 1998); and on the other hand the results of the ESS luminosity function analysis, which indicates that the three ESS spectral classes contain two additional components, dwarf elliptical and dwarf irregular galaxies, mixed into the intermediate and late-type classes respectively 
(de Lapparent et al. 2003). This leads us to separate the intermediate-type and late-type spectral classes into their giant and dwarf galaxy components, which we merge into two classes dominated by late spiral $(\mathrm{Sb}+\mathrm{Sc}+\mathrm{Sd} / \mathrm{Sm})$, and dwarf $(\mathrm{dE}+\mathrm{dI})$ galaxies respectively. The resulting three galaxy classes (earlytype, late spiral, dwarf galaxies) are therefore defined by spectral/morphological and luminosity criteria, which are both relevant for studying segregation effect in galaxy clustering. We use the corresponding Schechter and Gaussian luminosity functions for defining the selection function for each of the three galaxy types.

We test the various estimators of the correlation function, and adopt the Landy \& Szalay (1993) estimator with J3 weighting, which combines stability and minimum variance. The redshiftspace correlation function $\xi(s)$ can be fitted by a power-law with amplitude $s_{0}=7.49 \pm 3.18 h^{-1} \mathrm{Mpc}$ and slope $\gamma=0.90 \pm$ 0.13 in the interval $0.5<s<5 h^{-1}$ Mpc. At larger scales, $\xi(s)$ oscillates between negative and positive low amplitude values, with a peak at $\sim 35 h^{-1} \mathrm{Mpc}$ and its multiples. This is due to the combination of the pencil-beam geometry of the ESS survey with the alternation of walls and voids, as demonstrated by a pair separation analysis. The ESS also contains an over-dense region located in the redshift interval $0.41<z<0.44$, which affects the correlation function by adding excess large-scale power in $\xi(s)$. When removing this region, the power-law fit to $\xi(s)$ yields $s_{0}=4.22 \pm 1.15 h^{-1} \mathrm{Mpc}$ and $\gamma=1.22 \pm 0.15$ (in $0.5 \leq s \leq$ $5 h^{-1} \mathrm{Mpc}$ ), in better agreement with the other existing surveys.

We then calculate the redshift-space correlation function $\xi(s)$ for the three galaxy types. These show marked differences, with a dominant signal originating from the early-type galaxies at nearly all scales. The late spiral galaxies show a weaker correlation amplitude at small and large scales, in agreement with the type-density relationship (Blanton et al. 2005). The new result is that the dwarf galaxies show a very steep correlation function over a narrow range of scales: $\xi(s)$ decreases from the clustering amplitude of the early-type galaxies at very small scale, to more than one order of magnitude weaker at $r_{\mathrm{p}} \simeq 4 h^{-1} \mathrm{Mpc}$. These segregation effects in the two-point clustering quantify the visual impression drawn from the redshift cone of the ESS.

To free the correlation function measurements from the effect of peculiar velocities, as they decrease the clustering amplitude at small scales due to random motions and increase its amplitude at large scales due to coherent bulk flows, we then calculate the real-space correlation function as a function of projected separation $w\left(r_{\mathrm{p}}\right)$. This is done by integrating the 2-dimensional correlation function $\xi\left(r_{\mathrm{p}}, \pi\right)$ along the line-ofsight separation $\pi$. The resulting projected-separation correlation function $w\left(r_{\mathrm{p}}\right)$ can be adjusted by a power-law over a larger range of scales than $\xi(s)$, from $0.15 h^{-1} \mathrm{Mpc}$ to $10 h^{-1} \mathrm{Mpc}$. In this scale range, and after removing the over-density at $0.41<z<$ 0.44 , we obtain a best fit amplitude $r_{0}=3.50 \pm 1.21 h^{-1} \mathrm{Mpc}$ and a slope $\gamma=1.93 \pm 0.09$ which, as expected, is significantly steeper than that measured from $\xi(s)$.

When splitting the ESS by galaxy type, the projectedseparation correlation function $w\left(r_{\mathrm{p}}\right)$ shows similarities with $\xi(s)$, with again the early-type galaxies dominating over the other types at all scales. At variance with $\xi(s)$, the dwarf galaxies clustering dominates over the late spiral galaxies at $r_{\mathrm{p}} \simeq$ $0.15 \mathrm{~h}^{-1} \mathrm{Mpc}$. At larger scales, the dwarf galaxies have a spatial correlation function consistent with null clustering, whereas the late spiral galaxies take over at $r_{\mathrm{p}} \geq 0.6 \mathrm{~h}^{-1} \mathrm{Mpc}$, with a similar shape of the correlation function as for the early-type galaxies and a $50 \%$ lower amplitude.
Comparison of $\xi(s)$ and $w\left(r_{\mathrm{p}}\right)$ with and without inclusion of the over-dense region at $0.41<z<0.44$ provides useful clues on the nature of this region: the excess clustering appears entirely due to the fact that it contains richer and more densely clustered groups of galaxies than in the rest of the survey; and both the early-type and late spiral galaxies contribute to the excess clustering in the region. We then consider that the correlation functions for the ESS survey without the over-density are more representative of the overall galaxy distribution.

A subsequent analysis of the cross-correlation of the dwarf galaxies with the early-type and late spiral galaxies provides direct evidence that the dwarf galaxies are satellites of the giant galaxies. At $r_{\mathrm{p}} \leq 0.3 h^{-1} \mathrm{Mpc}$, pairs of early-type galaxies dominate over all the other types of pairs. Pairs of dwarf/early-type galaxies, dwarf galaxies, dwarf/late spiral galaxies, and late spiral galaxies are the next contributors to the small-scale two-point clustering, in decreasing order of contribution to the correlation function. In the intermediate scale range $0.3<r_{\mathrm{p}} \leq 5 h^{-1} \mathrm{Mpc}$, mixed pairs of dwarf and giant galaxies contribute equally to the clustering as pairs of giant galaxies. Then at $r_{\mathrm{p}} \simeq 10 \mathrm{~h}^{-1} \mathrm{Mpc}$, the clustering signal is dominated by pairs of giant galaxies. Moreover, the cross-correlation analysis indicates that at $r_{\mathrm{p}} \leq$ $0.3 h^{-1} \mathrm{Mpc}$, that dwarf and late spiral galaxies are not well mixed at $r_{\mathrm{p}} \leq 0.3 h^{-1} \mathrm{Mpc}$. Altogether, this suggests that the clustering of the dwarf galaxies around late spiral galaxies at $r_{\mathrm{p}} \leq 5 \mathrm{~h}^{-1} \mathrm{Mpc}$ may be an indirect consequence of how both galaxy types cluster in the environment of early-type galaxies.

We then interpret the variations in the correlation function with galaxy type in terms of membership to the underlying dark matter halos. This approach is eased by the separation into the giant and dwarf galaxies, as they exhibit a clear dichotomy in their halo components: the correlation function for the early-type galaxies shows a dip at $r_{\mathrm{p}} \simeq 1 h^{-1} \mathrm{Mpc}$, which is interpreted as the transition between the regimes in which the 1-halo and 2-halo pairs dominate resp., and both components show a significant contribution; in contrast, the dwarf and late spiral galaxy correlation functions are dominated by their 1-halo and 2-halo components, at small and large scales respectively. Altogether, this indicates that early-type galaxies tend to lie predominantly at the centers of the massive halos, whereas late spiral galaxies tend to lie either in the centers of less dense halos and/or in the outer regions of the densest halos. The small scale clustering is then not only determined by the dominant galaxies in the massive halos, but also by their dwarf satellites.

We also examine the two-point clustering for the bright and faint sub-samples of the early-type and late spiral galaxies. For both the early-type and late spiral galaxies, we detect a $1 \sigma$ excess clustering in the 2-halo regime of the bright sub-samples over the faint sub-samples, which is consistent with the expected properties of hierarchical clustering: the most massive halos have the strongest 2-halo clustering, and luminous galaxies are preferentially located within massive halos. Comparison of the 1-halo component brings the additional information that the relationship between halo mass and giant galaxy type is nearly independent of luminosity for the early-type galaxies, whereas faint late spiral galaxies might tend to reside in even less dense regions than their bright analogs.

At last, we compare our results with those from the other published analyses. Our power-law fits to $w\left(r_{\mathrm{p}}\right)$ for the full ESS sample, and for the sub-samples by galaxy type are consistent with those measured at comparable and lower redshifts from the other surveys. 


\section{Discussion and perspectives}

\subsection{The halo components of the correlation function}

One of the major results obtained here from the ESO-Sculptor redshift survey is that the projected-separation correlation function $w\left(r_{\mathrm{p}}\right)$ for each of the three galaxy types (early-type, late spiral and dwarf galaxies) presents marked deviations from a power-law, which can be interpreted as the transition between galaxies belonging to a same dark matter halo, and galaxies belonging to two different halos. This provides confirmation that the results obtained at low redshift $(z \lesssim 0.1)$ by Zehavi et al. (2005) and Magliocchetti \& Porciani (2003) extend to higher redshift $(z \lesssim 0.5)$. A similar result was recently obtained at even higher redshifts $(z \lesssim 1.2)$ based on the COMBO17 survey with broad and medium band photometric redshifts $(\sigma(z) / z \sim$ 0.01; Phleps et al. 2006). The ESS brings a useful confirmation based on spectroscopic redshifts, with $\sigma(z) \sim 0.00055$ at $0.1 \leq z \leq 0.51$.

In this context, the ESS results provide evidence in favor of the gravitational instability scenario for the formation of structure, in which the evolution of galaxy clustering is driven by the hierarchical merging of halos. Most recently, Conroy et al. (2006) have directly demonstrated that high-resolution dissipationless $\Lambda \mathrm{CDM}$ simulations can reproduce the observed bimodal behavior of the correlation function for absolute magnitudelimited samples at various redshift limits: these results are entirely based on combining the spatial clustering of the halos with a prescription that relates the galaxy luminosities to the maximum circular velocity of the sub-halos at the time of accretion. The hierarchical merging scenario is further validated by the detailed shape of the correlation functions for ESS early-type and late spiral galaxies, which allow a straightforward identification of the 1-halo and 2-halo components.

Direct modelling of the projected-separation correlation function using the "Halo Occupation Distribution" (HOD) (Benson et al. 2000; Berlind \& Weinberg 2002), or the more refined "Conditional Luminosity Function" approach (which takes into account the luminosity and colour distribution of galaxies within dark matter halos of varying mass; Yang et al. 2003), provides constraints on the halo parameters describing the central and satellite galaxies parameters (Magliocchetti \& Porciani 2003; Phleps \& Meisenheimer 2003; Abazajian et al. 2005; Zehavi et al. 2005; Cooray 2006). Nevertheless, several measurements of the correlation function at small scale also challenge the current version of the halo model for galaxy clustering: the very small scale clustering of luminous red galaxies from the SDSS is too steep and would require either a steeper dark halo profile or a galaxy distribution which is steeper than the dark matter at scales $0.01 \leq r_{\mathrm{p}} \leq 0.1 h^{-1} \mathrm{Mpc}$ (Masjedi et al. 2006). In contrast, Díaz et al. (2005) obtained projected density profiles of galaxy groups which are too flat compared to the standard Navarro et al. (1997) profile.

\subsection{Early-type versus late spiral segregation}

The second major result obtained from the ESS is the markedly different clustering properties of the two giant galaxy types, early-type and late spiral. Both types have comparable 2-halo components with a $50 \%$ higher amplitude for the early-type galaxies, whereas the 1-halo component of the early-type galaxies largely dominates over that for the late spiral galaxies. These results are remarkably similar to the predicted correlation functions calculated by Kauffmann et al. (1999a) in a $\Lambda$ CDM semi-analytical simulation, which exhibit a higher amplitude and steeper slope for the early-type/red galaxies, and a lower amplitude and a marked small-scale flattening at $r_{\mathrm{p}} \leq 1.0 \mathrm{~h}^{-1} \mathrm{Mpc}$ for the starforming galaxies.

In the framework of hierarchical clustering of the dark matter halos, our results imply a specific spatial segregation of the early-type and late spiral galaxies inside the dark matter halos, with the early-type galaxies residing in the center of the most massive halos, whereas the late spiral reside in their outskirts or in less dense halos. These segregation effects are consistent with the $50 \%$ higher pairwise velocity dispersion measured by Madgwick et al. (2003) for the 2dFGRS passive galaxies compared to the starforming galaxies, as it indicates that the passive galaxies inhabit preferentially the cores of high-mass virialized regions.

The link between the ESS type-segregation effects and the dark matter halos also finds direct confirmation from other recent analyses based on group catalogs. Zandivarez et al. (2003) and Yang et al. (2005c) show that the clustering properties of galaxy groups in the $2 \mathrm{dFGRS}$ match those of the dark matter halos in $\Lambda$ CDM N-body simulations. Yang et al. (2005b) thus derive a "halo-based" group finder algorithm which is optimized to associate a group to those galaxies which belong to the same dark matter halo. This allows one to directly examine the link between galaxies and their dark matter halos. Most interestingly, Yang et al. (2005c) separate the galaxy correlation function into the 1-group and 2-group components, and thus directly measure the individual 1-halo and 2-halo components.

The detected different distribution of ESS early-type and late spiral galaxies inside the dark matter halos raises the issue of whether the effect is due to each population belonging to different types of halos, or whether both galaxy types coexist within the same halos, but with a different spatial distribution. In the former case, the segregation effect would be related to the global halo properties like mass, whereas in the latter case, it would be related to the local properties such as dark matter density. The existence of both a 1-halo and 2-halo components in the cross-correlation function of the two ESS giant galaxy types (see Fig. 14) indicates that both effects may be at play. This is confirmed from the analyses performed with the 2dFGRS group catalogue of Yang et al. (2005b): on the one hand, Yang et al. (2005a) measure that central galaxies in high-mass, low-mass halos are mostly early-type, respectively late-type galaxies; on the other hand, Yang et al. (2005d) obtain direct evidence that early-type galaxies are closer to the luminosity-weighted group center than the late-type galaxies.

Another analysis of the three-dimensional density profiles of the 2dFGRS and SDSS groups found by a "friend-of-friend" algorithm indicates a type segregation, namely a decrease of the early-type galaxy fraction at larger group-centric distance and a corresponding increase of the late-type fraction (Díaz et al. 2005). At higher redshifts $(0.7 \leq z \leq 1.5)$, Coil et al. (2006) show that red galaxies are more centrally concentrated than blue galaxies in the galaxy groups extracted from the DEEP2 survey; this work uses yet another algorithm for group selection, based on the search for galaxy over-densities in redshift space which accounts for redshift-space distortions.

These various results find confirmation in the thorough analysis of Weinmann et al. (2006), based on the SDSS "halo-based" catalogue: the authors show that the fractions of early and late galaxy types not only vary with distance from the halo center, but also with halo mass over the full mass range probed, with more massive halos having higher/lower fractions of earlytype/late-type galaxies (Weinmann et al. 2006). Interestingly, 
Weinmann et al. (2006) find a flat distribution of intermediatetype galaxies as a function of mass and distance to the halo center, with types based on both color and specific star formation rate.

We also note that the detected segregation effects in the distribution of early-type and late-type galaxies within the dark matter halos are consistent with the type/density relationship, namely the trend for early-type galaxies to preferentially inhabit high-density regions. Although dense regions of a survey contain more galaxies that in the other regions, it is not obvious that they undergo stronger clustering. However, Abbas \& Sheth (2006) have shown that in high density regions of the SDSS, galaxies are more clustered than in low density regions, and this is valid at all scales from 0.1 to $30 \mathrm{~h}^{-1} \mathrm{Mpc}$. This remarkable property is interpreted by Abbas \& Sheth (2006) as providing strong support to the hierarchical models, as it is well reproduced by numerical and analytical models in which the entire effect is due to the correlation of galaxy properties with the mass of the parent halo, and to the fact that more massive halos populate dense regions.

The type-density relationship was clarified by the detailed study of Blanton et al. (2005), who found that color (present star formation rate) and luminosity (hence stellar mass, resulting from the history of past star formation) are the two properties most predictive of local density. Therefore, the detected segregation effects in the clustering of ESS galaxies for different spectral type (early-type versus late spiral) and luminosity (giant versus dwarf) are naturally expected. The uniqueness of the results presented here is that we identify for the first time the joint type/luminosity clustering segregation effect in terms of the very galaxy types which correspond to the locally well known morphological types.

\subsection{The dwarf galaxy correlation function}

The third major result obtained by the present ESS analysis is the correlation function for the dwarf galaxies. This function is measured for the first time, thanks to the separation of the dwarf galaxy component which the ESS allows, based on the type specific luminosity functions (de Lapparent et al. 2003). The projected-separation correlation function of the ESS dwarf galaxies can also be interpreted in terms of halo membership, as it displays a clear 1-halo component which falls off at $r_{\mathrm{p}}>0.3 \mathrm{~h}^{-1} \mathrm{Mpc}$. By their stronger clustering at all scales, the early-type galaxies appear as a key component of galaxy clustering. The auto-correlation function of the dwarf galaxies and their cross-correlation function with the early-type galaxies indicate that they are the next contributor to galaxy clustering at small scales. The additional evidence, based on the cross-correlation analysis, that dwarf and late spiral galaxies are not well mixed at scales $\leq 0.3 h^{-1} \mathrm{Mpc}$ leads to a picture in which dwarf galaxies are confined to the densest, hence central parts of the halos, and are preferentially satellites of early-type galaxies. This is in agreement with the observation that in local groups and clusters of galaxies, the dwarf-to-giant galaxy ratio is an increasing function of the richness of the galaxy concentration (Ferguson \& Sandage 1991; Trentham \& Hodgkin 2002; Trentham \& Tully 2002).

Most halo models parameterize the halo content in terms of a dominant galaxy and it satellites (Berlind et al. 2003; Cooray 2006). In their analysis of SDSS data, Zehavi et al. (2005) perform a two-component HOD modelling based on either red and blue central galaxies surrounded by red and blue satellite respectively. However, in the ESS, the dwarf galaxy sample is largely dominated by dwarf irregular hence blue galaxies, and these appear as satellites of the early-type hence red galaxies. Moreover, the late spiral galaxies may play the role of central galaxies in the less massive halos, whereas in the more massive halos, they may be considered as satellite galaxies. Another subtle effect is that detected by Weinmann et al. (2006) in the relative distribution of central and satellite galaxies in halos, which they name "galaxy conformity": for a halo of a given mass, the early-type fraction of satellites is significantly higher when the central galaxy is early-type rather than late-type. Altogether, these various results indicate that reality may be more complex than the simple two-component HOD models.

To illustrate the variety in galaxy types among a given halo, and their specific spatial distributions, let us consider our local group, which is typical of an intermediate-mass halo. It is dominated by the Milky Way, an Sb galaxy, and Andromeda, an Sab galaxy. In the ESS classification, the Milky Way would be classified as a late spiral, and Andromeda would be at the limit between early-type and late spiral. The third giant, although smaller galaxy, M33, is an Sdm galaxy, and would be classified as a late spiral. The blue Sm/Irr satellites of the Milky Way, the Magellanic Clouds, and the red dE satellites of Andromeda, M32 and M110, would all be classified as dwarf galaxies. There are in addition many $\mathrm{dSph}$ and $\mathrm{dI}$ galaxies in the local group, distributed at typically $0.05-0.1 h^{-1} \mathrm{Mpc}$ and $0.5 h^{-1} \mathrm{Mpc}$ from the giant galaxies (http://www.astro.washington.edu/ mayer/LG/LG.html).

\subsection{Perspectives}

The present analysis emphasizes the need for further studies of galaxy clustering as a function of galaxy type. This requires statistical analyses of large galaxy samples effectively containing halos within a large mass range, and a detailed knowledge of their galaxy content as a function of galaxy mass, luminosity and type. The specific clustering of the dwarf galaxies evidenced in the present ESS analysis suggests that probing the dark halo content in terms of the full sequence of giant and dwarf galaxy types would significantly enrich our understanding of galaxy clustering. Higher signal-to-noise measurement of the galaxy correlation functions for the various giant and dwarf galaxy types, and interpretation using the "Halo Occupation Distribution" or "Conditional Luminosity Function" would bring new insight into their distribution within the dark matter halos, and the relative role of the central and satellite galaxies in the halos.

The ultimate goal when identifying the different segregation effects which galaxies undergo in a given halo, is to make a link with the past history of star formation and mass accumulation of each system. In such studies, the definition of the galaxy types will be important, and the choice of the classification method will have an decisive impact. The innovative approach of programme EFIGI at IAP (see http: //terapix . iap. fr/), aimed at obtaining a quantitative morphological classification (Baillard et al., in preparation), should allow one to reliably classify large samples of galaxies, hence to better understand how each morphological type contributes to galaxy clustering.

Acknowledgements. We thank C. Benoist (OCA), A. Cappi (INAF, Osservatorio Astronomico di Bologna) and S. Maurogordato (OCA/CNRS) for useful discussions about subtleties of the two-point correlation function estimators. We also wish to express our thanks to F. Mignard (OCA/CNRS) who generously supplied the frequency mapping FAMOUS software (ftp://ftp.obs-nice.fr/pub/mignard/Famous) which has been used to produce the periodogram displayed in Fig. 6. 


\section{References}

Abazajian, K., Zheng, Z., Zehavi, I., et al. 2005, ApJ, 625, 613

Abbas, U., \& Sheth, R. K. 2006, MNRAS, 372, 1749

Andreon, S., \& Cuillandre, J.-C. 2002, ApJ, 569, 144

Arnouts, S., de Lapparent, V., Mathez, G., et al. 1997, A\&A, 124, 163

Baldi, A., Bardelli, S., \& Zucca, E. 2001, MNRAS, 324, 509

Baugh, C. M. 1996, MNRAS, 280, 267

Bellanger, C., \& de Lapparent, V. 1995, ApJ, 455, L103

Bellanger, C., de Lapparent, V., Arnouts, S., et al. 1995, A\&AS, 110, 159

Benoist, C., Maurogordato, S., da Costa, L. N., Cappi, A., \& Schaeffer, R. 1996, ApJ, 472, 452

Benson, A. J., Cole, S., Frenk, C. S., Baugh, C. M., \& Lacey, C. G. 2000, MNRAS, 311, 793

Benson, A. J., Frenk, C. S., Baugh, C. M., Cole, S., \& Lacey, C. G. 2001, MNRAS, 327, 1041

Berlind, A. A., \& Weinberg, D. H. 2002, ApJ, 575, 587

Berlind, A. A., Weinberg, D. H., Benson, A. J., et al. 2003, ApJ, 593, 1

Bernstein, G. M. 1994, ApJ, 424, 569

Binggeli, B., Tarenghi, M., \& Sandage, A. 1990, A\&A, 228, 42

Blanton, M. R., Eisenstein, D., Hogg, D. W., Schlegel, D. J., \& Brinkmann, J. 2005, ApJ, 629, 143

Brainerd, T. G., Smail, I., \& Mould, J. 1995, MNRAS, 275, 781

Bromley, B. C., Press, W. H., Lin, H., \& Kirshner, R. P. 1998, ApJ, 505, 25

Budavári, T., Connolly, A. J., Szalay, A. S., et al. 2003, ApJ, 595, 59

Carlberg, R. G., Yee, H. K. C., Morris, S. L., et al. 2000, ApJ, 542, 57

Coil, A. L., Davis, M., Madgwick, D. S., et al. 2004, ApJ, 609, 525

Coil, A. L., Gerke, B. F., Newman, J. A., et al. 2006, ApJ, 638, 668

Cole, S., Ellis, R. S., Broadhurst, T. J., \& Colless, M. M. 1994, MNRAS, 267, 541

Colless, M., Dalton, G., Maddox, S., et al. 2001, MNRAS, 328, 1039

Conroy, C., Wechsler, R. H., \& Kravtsov, A. V. 2006, ApJ, 647, 201

Cooray, A. 2006, MNRAS, 365, 842

Courteau, S., \& van den Bergh, S. 1999, AJ, 118, 337

Davis, M., \& Huchra, J. 1982, ApJ, 254, 437

Davis, M., \& Peebles, P. J. E. 1983, ApJ, 267, 465

de Lapparent, V., Geller, M. J., \& Huchra, J. P. 1986, ApJ, 302, L1

de Lapparent, V., Geller, M. J., \& Huchra, J. P. 1988, ApJ, 332, 44

de Lapparent, V., Galaz, G., Bardelli, S., \& Arnouts, S. 2003, A\&A, 404, 831

de Lapparent, V., Arnouts, S., Galaz, G., \& Bardelli, S. 2004, A\&A, 422, 841

Díaz, E., Zandivarez, A., Merchán, M. E., \& Muriel, H. 2005, ApJ, 629, 158

Dressler, A. 1980, ApJ, 236, 351

Efstathiou, G. 1988, in Comets to Cosmology, LNP, 297, 312

Ekholm, T., Baryshev, Y., Teerikorpi, P., Hanski, M. O., \& Paturel, G. 2001, A\&A, 368, L17

Ferguson, H. C., \& Sandage, A. 1991, AJ, 101, 765

Fioc, M., \& Rocca-Volmerange, B. 1997, A\&A, 326, 950

Fisher, K. B., Davis, M., Strauss, M. A., Yahil, A., \& Huchra, J. 1994, MNRAS, 266,50

Folkes, S. R., Lahav, O., \& Maddox, S. J. 1996, MNRAS, 283, 651

Galaz, G., \& de Lapparent, V. 1998, A\&A, 332, 459

Giuricin, G., Samurović, S., Girardi, M., Mezzetti, M., \& Marinoni, C. 2001, ApJ, 554, 857

Grant, N. I., Kuipers, J. A., \& Phillipps, S. 2005, MNRAS, 363, 1019

Guzzo, L., Bartlett, J. G., Cappi, A., et al. 2000, A\&A, 355, 1

Hamilton, A. J. S. 1993, ApJ, 417, 19

Hawkins, E., Maddox, S., Cole, S., et al. 2003, MNRAS, 346, 78

Hermit, S., Santiago, B. X., Lahav, O., et al. 1996, MNRAS, 283, 709

Hogg, D. W., Cohen, J. G., \& Blandford, R. 2000, ApJ, 545, 32

Jenkins, A., Frenk, C. S., White, S. D. M., et al. 2001, MNRAS, 321, 372

Jerjen, H., \& Tammann, G. A. 1997, A\&A, 321, 713

Jing, Y. P., Mo, H. J., \& Boerner, G. 1998, ApJ, 494, 1

Kauffmann, G., Colberg, J. M., Diaferio, A., \& White, S. D. M. 1999a, MNRAS, 303,188

Kauffmann, G., Colberg, J. M., Diaferio, A., \& White, S. D. M. 1999b, MNRAS, 307,529

Kennicutt, R. C. 1992, ApJS, 79, 255

Kerscher, M., Szapudi, I., \& Szalay, A. S. 2000, ApJ, 535, L13

Landy, S. D., \& Szalay, A. S. 1993, ApJ, 412, 64

Le Fevre, O., Hudon, D., Lilly, S. J., et al. 1996, ApJ, 461, 534
Li, C., Kauffmann, G., Jing, Y. P., et al. 2006, MNRAS, 368, 21

Lilly, S. J., Tresse, L., Hammer, F., Crampton, D., \& Le Fevre, O. 1995, ApJ, 455,108

Lin, H., Yee, H. K. C., Carlberg, R. G., et al. 1999, ApJ, 518, 533

Ling, E. N., Barrow, J. D., \& Frenk, C. S. 1986, MNRAS, 223, 21P

Loveday, J., Efstathiou, G., Peterson, B. A., \& Maddox, S. J. 1992, ApJ, 400, L43

Loveday, J., Maddox, S. J., Efstathiou, G., \& Peterson, B. A. 1995, ApJ, 442, 457

Loveday, J., Tresse, L., \& Maddox, S. 1999, MNRAS, 310, 281

Madgwick, D. S., Hawkins, E., Lahav, O., et al. 2003, MNRAS, 344, 847

Magliocchetti, M., \& Porciani, C. 2003, MNRAS, 346, 186

Masjedi, M., Hogg, D. W., Cool, R. J., et al. 2006, ApJ, 644, 54

Maurogordato, S., Schaeffer, R., \& da Costa, L. N. 1992, ApJ, 390, 17

Meneux, B., Le Fèvre, O., Guzzo, L., et al. 2006, A\&A, 452, 387

Navarro, J. F., Frenk, C. S., \& White, S. D. M. 1997, ApJ, 490, 493

Norberg, P., Baugh, C. M., Hawkins, E., et al. 2002, MNRAS, 332, 827

Park, C., Vogeley, M. S., Geller, M. J., \& Huchra, J. P. 1994, ApJ, 431, 569

Pearce, F. R., Jenkins, A., Frenk, C. S., et al. 2001, MNRAS, 326, 649

Peebles, P. J. E. 1980, The large-scale structure of the universe, Research supported by the National Science Foundation (Princeton, N.J.: Princeton University Press), 435

Perlmutter, S., Aldering, G., Goldhaber, G., et al. 1999, ApJ, 517, 565

Phillips, J., Weinberg, D. H., Croft, R. A. C., et al. 2001, ApJ, 560, 15

Phleps, S., \& Meisenheimer, K. 2003, A\&A, 407, 855

Phleps, S., Peacock, J. A., Meisenheimer, K., \& Wolf, C. 2006, A\&A, 457, 145

Pollo, A., Guzzo, L., Le Fèvre, O., et al. 2006, A\&A, 451, 409

Pons-Bordería, M., Martínez, V. J., Stoyan, D., Stoyan, H., \& Saar, E. 1999, ApJ, 523,480

Postman, M., \& Geller, M. J. 1984, ApJ, 281, 95

Ramella, M., Pisani, A., \& Geller, M. J. 1997, AJ, 113, 483

Ratcliffe, A., Shanks, T., Broadbent, A., et al. 1996, MNRAS, 281, L47

Ratcliffe, A., Shanks, T., Parker, Q. A., et al. 1998a, MNRAS, 300, 417

Ratcliffe, A., Shanks, T., Parker, Q. A., \& Fong, R. 1998b, MNRAS, 296, 173

Riess, A. G., Filippenko, A. V., Challis, P., et al. 1998, AJ, 116, 1009

Saunders, W., Rowan-Robinson, M., \& Lawrence, A. 1992, MNRAS, 258, 134

Schechter, P. 1976, ApJ, 203, 297

Shectman, S. A., Landy, S. D., Oemler, A., et al. 1996, ApJ, 470, 172

Shepherd, C. W., Carlberg, R. G., Yee, H. K. C., et al. 2001, ApJ, 560, 72

Small, T. A., Sargent, W. L. W., \& Hamilton, D. 1997, ApJ, 111, 1

Small, T. A., Ma, C., Sargent, W. L. W., \& Hamilton, D. 1999, ApJS, 524, 31

Smoot, G. F., Bennett, C. L., Kogut, A., et al. 1991, ApJ, 371, L1

Tonry, J. L., Schmidt, B. P., Barris, B., et al. 2003, MNRAS, 594, 1

Trentham, N. 1997, MNRAS, 286, 133

Trentham, N., \& Hodgkin, S. 2002, MNRAS, 333, 423

Trentham, N., \& Tully, R. B. 2002, MNRAS, 335, 712

Tucker, D. L., Oemler, A., Kirshner, R. P., et al. 1997, MNRAS, 285, L5

Weinberg, S. 1972, Gravitation and cosmology: Principles and applications of the general theory of relativity (New York: Wiley)

Weinmann, S. M., van den Bosch, F. C., Yang, X., \& Mo, H. J. 2006, MNRAS, 366,2

Willmer, C. N. A., da Costa, L. N., \& Pellegrini, P. S. 1998, AJ, 115, 869

Yahil, A., Tammann, G. A., \& Sandage, A. 1977, ApJ, 217, 903

Yang, X., Mo, H. J., \& van den Bosch, F. C. 2003, MNRAS, 339, 1057

Yang, X., Mo, H. J., Jing, Y. P., van den Bosch, F. C., \& Chu, Y. 2004, MNRAS, 350,1153

Yang, X., Mo, H. J., Jing, Y. P., \& van den Bosch, F. C. 2005a, MNRAS, 358, 217

Yang, X., Mo, H. J., van den Bosch, F. C., \& Jing, Y. P. 2005b, MNRAS, 356, 1293

Yang, X., Mo, H. J., van den Bosch, F. C., \& Jing, Y. P. 2005c, MNRAS, 357, 608

Yang, X., Mo, H. J., van den Bosch, F. C., et al. 2005d, MNRAS, 362, 711

Yoshida, N., Colberg, J., White, S. D. M., et al. 2001, MNRAS, 325, 803

Zandivarez, A., Merchán, M. E., \& Padilla, N. D. 2003, MNRAS, 344, 247

Zehavi, I., Blanton, M. R., Frieman, J. A., et al. 2002, ApJ, 571, 172

Zehavi, I., Weinberg, D. H., Zheng, Z., et al. 2004, ApJ, 608, 16

Zehavi, I., Zheng, Z., Weinberg, D. H., et al. 2005, ApJ, 630, 1

Zheng, Z., Tinker, J. L., Weinberg, D. H., \& Berlind, A. A. 2002, ApJ, 575, 617 
V. de Lapparent and E. Slezak: ESO-Sculptor clustering by galaxy type at $z \simeq 0.1-0.5$, Online Material $p 1$

\section{Online Material}




\section{Appendix A: Distances}

Redshifts $z$ are converted into comoving distances $r$ from the observer by using $\tau$ the dimensionless radial comoving coordinate of the Robertson-Walker line element (Weinberg 1972):

$r=\frac{c}{H_{0}} \tau$

with

$\tau=\int_{0}^{z}\left[\Omega_{\mathrm{m}}(1+v)^{3}+\Omega_{\Lambda}\right]^{-\frac{1}{2}} \mathrm{~d} v$.

Given the chosen flat geometry, the comoving separation between any two objects $i$ and $j$ with angular separation $\theta$ on the sky is expressed from the usual law of cosines as:

$s \equiv s_{i j}=\frac{c}{H_{0}} \sqrt{\tau_{i}^{2}+\tau_{j}^{2}-2 \tau_{i} \tau_{j} \cos \theta}$.

\section{Appendix B: Estimators}

The galaxy-galaxy correlation function in redshift space $\xi(s)$ is defined as the probability in excess of a homogeneous Poisson distribution of finding in any direction two galaxies at distance $s$ from each other:

$\delta P=\rho[1+\xi(s)] \delta V$,

where $\rho$ is the mean space number density of galaxies and $\delta V$ is the volume element (see Peebles 1980). When the distribution is homogeneous, $\xi(s) \equiv 0$, but any uncertainty in the mean density of the galaxy sample $\rho$ under study may result in an error in the correlation amplitude, especially at large spatial scales where the signal is below the fractional uncertainty in the density. To overcome this difficulty, together with the problem of selection and boundaries effects in the data sample, several estimators have been introduced which allow one to measure $\xi(s)$ from a finite set of objects with minimum bias and variance. They are generally defined as suitably normalized ratios of counts of galaxy pairs separated by distance $s$ in a narrow interval of distances $\delta s$ centered on $s$. The various considered pair counts are: (i) the weighted number of pairs of observed galaxies

$D D(s)=\Sigma_{i, j>i} w_{i}^{(\mathrm{d})} w_{j}^{(\mathrm{d})} ;$

(ii) the weighted number of pairs for a computer-generated random distribution with the same selection criteria as the galaxy sample (see Appendix E)

$R R(s)=\Sigma_{i, j>i} w_{i}^{(\mathrm{r})} w_{j}^{(\mathrm{r})} ;$

and (iii) the weighted number of pairs between the set of random objects and the observed galaxies

$D R(s)=\Sigma_{i, j>i} w_{i}^{(\mathrm{d})} w_{j}^{(\mathrm{r})}$.

Note that a given pair of objects $(i, j)$ is only counted once in Eqs. (B.2)-(B.4). The adopted functions for the weights $w_{i}^{\mathrm{d}}$ and $w_{i}^{\mathrm{r}}$ are discussed in Appendix D.

In the following, we also denote $N_{\mathrm{d}}$ the number of observed galaxies in the data sample and $N_{\mathrm{r}}$ the number of points in the corresponding random set (see Appendix E).

For investigating the correlation properties of the ESOSculptor redshift survey, we consider three of the demonstrated best estimators of $\xi(s)$. The first estimator is that by Davis \& Peebles (1983, denoted DP estimator hereafter). If we denote
$C^{(\mathrm{d})}$ and $C^{(\mathrm{r})}$ the weighted object counts in the galaxy and random samples resp. (see Appendix E for the definition of these quantities), this estimator may be defined for large enough samples $\left(N_{\mathrm{d}}, N_{\mathrm{r}} \gg 1\right)$ as:

$1+\hat{\xi}_{\mathrm{DP}}(s) \simeq 2 \frac{C^{(\mathrm{r})}}{C^{(\mathrm{d})}} \frac{D D(s)}{D R(s)}$,

with the sums in the pair counts extending over all independent pairs with redshift-space separations between $s-\delta s / 2$ and $s+\delta s / 2 ; \hat{x}$ is the standard notation to refer to an estimator of quantity $x$. The DP estimator is poorly sensitive to the adopted edge correction but its variance varies as $1 / \rho$. With its quadratic dependence on the uncertainty in the mean density $\rho$, the Hamilton (1993) estimator (denoted H estimator hereafter) performs better than the DP estimator for sparse samples with a poorly determined mean density. The $\mathrm{H}$ estimator takes into account the pair count within the random sample according to:

$1+\hat{\xi}_{\mathrm{H}}(s) \simeq 4 \frac{D D(s) \times R R(s)}{[D R(s)]^{2}}$,

which includes thereby a measure of the relative densities of the two catalogues at any separation, via the pair counts (independent pairs only); this allows one to bypass the density normalization factor present in Eq. (B.5).

To minimize the effects of the finite solid angle on the sky, Landy \& Szalay (1993) introduced yet another quadratic estimator, denoted LS estimator hereafter:

$1+\hat{\xi}_{\mathrm{LS}}(s) \simeq 2+\left[\frac{C^{(\mathrm{r})}}{C^{(\mathrm{d})}}\right]^{2} \frac{D D(s)}{R R(s)}-\frac{C^{(\mathrm{r})}}{C^{(\mathrm{d})}} \frac{D R(s)}{R R(s)} ;$

again, only independent pairs are counted. The authors show that this estimator performs very well with a nearly Poisson variance for uncorrelated data (for other clustering regimes, see Bernstein 1994), and is less sensitive to the number of points in the random distribution than the $\mathrm{H}$ estimator (Kerscher et al. 2000). Each estimator has its own theoretical advantages and weak points which depend on the scale range under study (Pons-Bordería et al. 1999). Even if recent analyses have shown that the 3 estimators agree within the error bars (Tucker et al. 1997; Guzzo et al. 2000; Zehavi et al. 2002; but see Loveday et al. 1995), here we choose to calculate the 3 estimators for each ESS sub-sample and to compare the estimates. This allows us to secure our conclusions on the behavior of the correlation function.

Finally, we define the cross-correlation function between two different sub-samples. It measures the excess probability over random of finding a galaxy belonging to sample \#2 at a separation $s$ from a galaxy belonging to sample \#1. The same estimators as for the auto-correlation function, but with slightly modified expressions and normalization factors can be used. The DP, $\mathrm{H}$ and LS estimators for the two-point cross-correlation can be written respectively as:

$$
\begin{aligned}
1+\hat{\xi}_{\mathrm{DP}}(s) & \simeq \sqrt{\frac{C^{\left(\mathrm{r}_{1}\right)} C^{\left(\mathrm{r}_{2}\right)}}{C^{\left(\mathrm{d}_{1}\right)} C^{\left(\mathrm{d}_{2}\right)}}} \frac{D_{1} D_{2}(s)}{\sqrt{D_{1} R_{2}(s) D_{2} R_{1}(s)}}, \\
1+\hat{\xi}_{\mathrm{H}}(s) \simeq & \frac{D_{1} D_{2}(s) \times R_{1} R_{2}(s)}{D_{1} R_{2}(s) D_{2} R_{1}(s)}, \\
1+\hat{\xi}_{\mathrm{LS}}(s) \simeq 2 & +\frac{C^{\left(\mathrm{r}_{1}\right)} C^{\left(\mathrm{r}_{2}\right)}}{C^{\left(\mathrm{d}_{1}\right)} C^{\left(\mathrm{d}_{2}\right)}} \frac{D_{1} D_{2}(s)}{R_{1} R_{2}(s)} \\
& -\frac{C^{\left(\mathrm{r}_{1}\right)}}{C^{\left(\mathrm{d}_{1}\right)}} \frac{D_{1} R_{2}(s)}{R_{1} R_{2}(s)}-\frac{C^{\left(\mathrm{r}_{2}\right)}}{C^{\left(\mathrm{d}_{2}\right)}} \frac{D_{2} R_{1}(s)}{R_{2} R_{1}(s)},
\end{aligned}
$$


where the $D_{1} D_{2}(s), R_{1} R_{2}(s)$ sums are the weighted numbers of all pairs with separations $s \pm \delta s / 2$, and the $D_{1} R_{2}(s), D_{2} R_{1}(s)$ sums are the weighted numbers of all cross-reference datarandom and random-data pairs. We have checked that the three estimators in Eqs. (B.8) to (B.10) yield consistent measures for the various cross-correlation functions considered in Sect. 5.4.

\section{Appendix C: Selection functions}

In magnitude-limited surveys, the observed galaxy density varies strongly with distance $r$ from the origin, because such surveys do not include all the galaxies within a limiting redshift distance, but only those bright enough to be detected. To calculate the correlation functions, one must account for this selection effect. The corresponding selection function is derived from the galaxy luminosity function, denoted $\phi(M)$. The probability that a galaxy at comoving distance $r$ (see Appendix A) with absolute magnitude $M$ is detected in a sample can be written as:

$p(M)=\frac{\phi(M)}{\int_{M_{\text {bright }}(r)}^{M_{\text {faint }}(r)} \phi(M) \mathrm{d} M}$,

where $M_{\text {bright }}(r)$ and $M_{\text {faint }}(r)$ are the brightest and faintest absolute magnitudes observable at distance $r$. The selection function $\psi(r)$ is then defined as the ratio between the number of the detectable objects at $r$ and the total number of galaxies which would be observed in a homogeneous sample between absolute magnitudes $M_{1}$ to $M_{2}$ :

$\psi(r)=\frac{\int_{\max \left(M_{\text {bright }}(r), M_{1}\right)}^{\min \left(M_{\text {faint }}(r), M_{2}\right)} \phi(M) \mathrm{d} M}{\int_{M_{1}}^{M_{2}} \phi(M) \mathrm{d} M}$.

Note that $\psi(r)$ takes values in the interval $[0,1]$, with $\psi(0)=1$ and $\psi(r) \rightarrow 0$ when $r \rightarrow \infty$.

Here, we use for $M_{1}$ and $M_{2}$ the effective boundaries (rounded to the first decimal place) of each considered subsample, in order to have the same distribution in absolute magnitude for the observed sample and the comparison random set (see Appendix E). The values of $M_{1}$ and $M_{2}$ adopted for each considered ESS sub-sample are listed in the third column of Table 1. In the case of a sub-sample with a cut in absolute magnitude, $M_{1}$ or $M_{2}$ in Eq. (C.2) are replaced with the appropriate bound.

Because only $92 \%$ of the ESS galaxies with $R_{\mathrm{c}} \leq 20.5$, and $52 \%$ with $R_{\mathrm{c}} \leq 21.5$ have a measured redshift, we also include the redshift incompleteness in the calculation of the selection function. As the redshift incompleteness is uncorrelated with the position on the sky and only depends on the apparent magnitude (see de Lapparent et al. 2003), we proceed as follows. We bin the redshift incompleteness in fixed intervals of $0.5 \mathrm{mag}$ in apparent magnitude. At each comoving distance $r$, we calculate the corresponding intervals in absolute magnitude and split the numerator integral in Eq. (C.2) into sub-integrals using these intervals; then in each sub-integral, the incompleteness is accounted for as a constant factor $\leq 1$.

Combined sub-samples including more than one class have their selection function defined as follows. The expected distance distribution for a homogeneous distribution with a single spectral class is

$N(r)=\phi_{0} \psi(r) \int_{M_{1}}^{M_{2}} \varphi(M) \mathrm{d} M$

where $\varphi(M)$ is the shape of the luminosity function, defined as $\phi(M) \mathrm{d} M=\phi_{0} \varphi(M) \mathrm{d} M$ for a Gaussian parameterization. We also use Eq. (C.4) for the composite luminosity functions of the ESS intermediate-type and late-type samples (see Sect. 2.2); then, the shape of the additive Schechter component contributed to $\varphi(M)$ is scaled by $\phi^{*} / \phi_{0}$.

By equating the total expected number of galaxies to the sum of the expected numbers for each sub-sample, we obtain

$\psi(r)=\frac{\sum_{k=1}^{K} \psi_{k}(r) \phi_{0 k} \int_{M_{1}}^{M_{2}} \varphi_{k}(M) \mathrm{d} M}{\phi_{0} \int_{M_{1}}^{M_{2}} \varphi(M) \mathrm{d} M}$

The integral in the denominator is unknown, as the parametric form of the luminosity function corresponding to the total sample is a priori unknown. It can however be determined using the boundary condition that Eq. (C.5) must also be valid at $r=0$, where all selection functions $\psi(r)$ and $\psi_{k}(r)$ are equal to unity (see Eq. (C.2)). This yields

$\psi(r)=\frac{\sum_{k=1}^{K} \psi_{k}(r) \phi_{0 k} \int_{M_{1}}^{M_{2}} \varphi_{k}(M) \mathrm{d} M}{\sum_{k=1}^{K} \phi_{0 k} \int_{M_{1}}^{M_{2}} \varphi_{k}(M) \mathrm{d} M}$.

For ESS sub-samples with a cut in absolute magnitude, the selection function for a single spectral class (Eq. (C.2)) is calculated with the modified values of the bounds $M_{1}$ and $M_{2}$. For ESS sub-samples from which is extracted a redshift interval, the selection function is set to zero in that interval. For both types of cuts, the selection function for a combined sample is derived using Eq. (C.6).

\section{Appendix D: Weights}

The selection functions described in Appendix $\mathrm{C}$ can be accounted for in the calculation of correlation functions by weighting each pair of galaxies in the various estimators of Appendix B according to three different schemes. When the weighting function is constant

$w(r) \equiv 1$

(denoted "equal pair" weighting), pairwise estimates of the correlation function are biased against the few distant galaxies. In contrast, weighting the galaxies in proportion to the inverse radial selection function $\psi(r)$

$w(r) \equiv 1 / \psi(r)$

(denoted "equal volume" weighting) gives too small a weight to the well-sampled nearby regions where clustering dominates the galaxy shot noise. This leads to the introduction of the minimum-variance weighting scheme in which each object at distance $r$ in a pair with separation $s$ is applied a weight

$w(r, s)=1 /\left[1+4 \pi \rho \psi(r) J_{3}(s)\right]$,

where $4 \pi J_{3}(s)$ is the volume integral of the two-point correlation function $\xi(s)$ out to a separation $s$; note that this approach can only be used if $\xi(s)$ vanishes on scales larger than some scale $s_{\mathrm{c}}$. The minimum-variance weighting (also denoted $J 3$ weighting) is intermediate between the two other weighting schemes: although $\psi(r)$ increases at small values of $r$, the decrease of $J 3$ with $r$ dominates and $4 \pi \rho \psi(r) J_{3}(s) \ll 1$ at small $r$, so that $w(r, s) \sim 1$; in contrast, at large values of $r, 4 \pi \rho \psi(r) J_{3}(s) \gg 1$ and $w(r, s)$ behaves as $1 / \psi(r)$.

It was also shown that the $J 3$ weighting scheme gives the minimum uncertainty in the clustering amplitude on scales 
where $\xi(s) \leq 1$ (Efstathiou 1988; Saunders et al. 1992). Equation (D.3) results from a separable approximation of the true minimum-variance pair weighting which is valid in the linear regime applicable at large separations, when higher-order statistics can be neglected (Hamilton 1993). The integral $J_{3}(s)$ can be calculated without involving any iterative technique by modeling the required correlation function with a power-law model and still yield accurate estimates of $\xi(s)$, especially if one uses unbiased estimators for $\xi(s)$ (see Appendix B; see also Ratcliffe et al. 1998b; Guzzo et al. 2000).

Here, we estimate $J_{3}(s)$ using the power-law model which provides a good fit to most observed samples

$\xi(s)=\left(s / s_{0}\right)^{-\gamma}$

with

$$
\begin{aligned}
& \gamma=1.6 \\
& s_{0}=6 h^{-1} \mathrm{Mpc}
\end{aligned}
$$

as measured from the existing redshift surveys (de Lapparent et al. 1988; Maurogordato et al. 1992; Loveday et al. 1995; Hermit et al. 1996; Tucker et al. 1997; Willmer et al. 1998; Ratcliffe et al. 1998a; Zehavi et al. 2002; Hawkins et al. 2003). A posteriori, this is also in acceptable agreement with the results for the ESS (see Eq. (11)). The power-law parameterization of Eq. (D.4) is used only for separations smaller than $s_{\mathrm{c}}=30 \mathrm{~h}^{-1} \mathrm{Mpc}$; we use $\xi(s)=0$ otherwise. This yields

$$
\begin{array}{ll}
J_{3}(s)=12.6 s^{1.4} h^{-3} \mathrm{Mpc}^{3} & \text { for } \quad s \leq s_{\mathrm{c}}, \\
J_{3}(s)=1468 h^{-3} \mathrm{Mpc}^{3} & \text { for } \quad s>s_{\mathrm{c}} .
\end{array}
$$

Note that the $J_{3}$ weighting favors low-luminosity pairs at small separations while luminous objects dominate the estimate on large scales (Guzzo et al. 2000). The overall shape of the correlation function may then change in case of any luminosity dependence of the galaxy clustering. The $J_{3}$ weighting results must therefore be compared with those obtained in the "equal pair" weighting, in particular at small scales.

\section{Appendix E: Normalization}

Because the two-point correlation measures the excess number of pairs over a homogeneous distribution, it requires a normalization which is obtained by comparison of the number of pairs in a given ESS sample, with that derived from a mock homogeneous distribution occupying the same volume as the ESS sample, and having the selection function $\psi(r)$ derived from the luminosity function of that sample. Because the redshift incompleteness is accounted for in the selection function $\psi(r)$ (see Appendix C), it is automatically accounted for in the random distributions, and does not need to be included into the weighting functions of Eqs. (D.1)-(D.3).

The Monte-Carlo set containing $N_{\mathrm{r}}$ points, and corresponding to each data sub-sample defined in Table 2 includes at least fifty times as many objects as the observational catalogue:

$$
\begin{array}{lll}
N_{\mathrm{r}}=50 N_{\mathrm{d}} & \text { for } & N_{\mathrm{d}} \geq 100, \\
N_{\mathrm{r}}=5000 & \text { for } & N_{\mathrm{d}}<100 .
\end{array}
$$

The number of random points $N_{\mathrm{r}}$ is then large enough to ensure that the fluctuation in $C^{(\mathrm{r})}$ (see below) and the related uncertainty in $\xi(s)$ are negligible, so that the uncertainties in the correlation function are dominated by those in the pair count $D D(s)$ (see Eq. (B.2)). The normalizing factor $C^{(\mathrm{r})} / C^{(\mathrm{d})}$ (defined below) then allows one to normalize the density of the random distribution to that of the data sample for the DP and LS estimators
(Eqs. (B.5) and (B.7); in the $\mathrm{H}$ estimator of Eq. (B.6), the normalizing factor cancels out). This normalization of the observed number of pairs is equivalent to adopting the mean density for each observed sample as the reference density.

Each random distribution is then generated by randomly drawing points with a redshift probability distribution defined by the selection function $\psi(r)$ corresponding to the data sub-sample (Eq. (C.2)). The RA and Dec coordinates of each random point are also drawn randomly between the ESS extreme values while accounting for the small excluded RA and Dec regions due to saturated stars. For a sub-sample in Table 1 which is based on one spectral type and has cuts in either redshift or absolute magnitude, the number of observed objects $N_{\mathrm{d}}$ after applying the redshift or magnitude cut is listed in Table 1 and used in Eq. (E.1), and the random distributions is generated using the cut-updated selection function for that spectral type (see Appendix C). For the combined samples (for example the sample containing "all galaxies"), we use the reunion of the random sets corresponding to each spectral-type sub-sample and each satisfying Eq. (E.1), which ensures that the selection functions and relative proportions for each spectral-type are taken into account.

Then, from each observed sample and its corresponding random set, we calculate the data pairs counts $D D(s)$ (Eq. (B.2)) and the comparison random pair counts $D R(s)$ and $R R(s)$ (Eqs. (B.4) and (B.3)). In the case of "equal pair" or "equal volume" weighting, the normalizing ratio of weighted counts of objects which appear in the DP and LS estimators (see Eqs. (B.5) and (B.7)) is defined as

$$
\frac{C^{(\mathrm{r})}}{C^{(\mathrm{d})}}=\frac{\sum_{l=1}^{N_{\mathrm{r}}} w\left(r_{l}\right)}{\sum_{k=1}^{N_{\mathrm{d}}} w\left(r_{k}\right)}
$$

where the sums run over the $N_{\mathrm{r}}, N_{\mathrm{d}}$ objects of the random, resp. observed distributions, and the weights are defined in Eqs. (D.2) and (D.3). In the case of $J_{3}$ weighting, the ratio of weighted pair counts in the DP and LS estimators is computed as

$$
\frac{C^{(\mathrm{r})}}{C^{(\mathrm{d})}}=\frac{\sum_{l=1}^{N_{\mathrm{r}}} w\left(r_{l}, s_{\mathrm{c}}\right)}{\sum_{k=1}^{N_{\mathrm{d}}} w\left(r_{k}, s_{\mathrm{c}}\right)} \quad \text { with } \quad s_{\mathrm{c}} \equiv 30 h^{-1} \mathrm{Mpc} .
$$

By fixing the value of the $J 3$ weights at the large pair separation $s_{\mathrm{c}}=30 h^{-1} \mathrm{Mpc}$, we ensure that the weighted pair counts $C^{(\mathrm{d})}$ and $C^{(\mathrm{r})}$ are not affected by galaxy clustering.

\section{Appendix F: Mean density}

In the case of the $J_{3}$ pair-weighting function (Eq. (D.3)), one must define an estimator of the mean number density $\rho$. Given a magnitude-limited sample of $N$ galaxies, we denote $\rho\left(M_{1}<\right.$ $\left.M<M_{2}\right)$ the mean density of galaxies with absolute magnitude $M$ in the interval $M_{1}<M<M_{2}$ (corresponding to the bounds used in the selection function, in Eq. (C.2)). An estimator of $\rho\left(M_{1}<M<M_{2}\right)$ which is unbiased by the selection function $\psi(r)$ can be obtained using

$\hat{\rho}\left(M_{1}<M<M_{2}\right)=\frac{\sum_{i=1}^{N} w\left(r_{i}\right)}{\int_{r_{\text {min }}}^{r_{\max }} w(r) \psi(r) \frac{\mathrm{d} V}{\mathrm{~d} r} \mathrm{~d} r}$,

where $w(r)$ is a weighting function (see Eqs. (D.1)-(D.3)). The comoving distances $r_{\min }$ and $r_{\max }$ correspond to the redshift boundaries of the sample (Eq. (1)).

Davis \& Huchra (1982) showed that in the case of "equal pair" weighting (Eq. (D.1)), this estimator is the most stable, 
but heavily weights galaxies near the peak of the redshift distribution. In the case of "equal volume" weighting (Eq. (D.2)), Davis \& Huchra (1982) also showed that Eq. (F.1) is close to the minimum variance estimator, but that it heavily weights distant structures. The $J_{3}$ weighting defined in Eq. (D.3) provides an intermediate estimate of the mean density.

To estimate the mean density required for a $J_{3}$ pair-weighting (Eq. (D.2)), we iterate over Eqs. (F.1) and (D.3). The "equal volume" weighting (Eq. (D.1)) is used for calculating a first value of $\rho$. Then, in each calculation of $w(r)$, we use Eq. (D.3) with $J_{3}(s) \equiv J_{3}\left(30 h^{-1} \mathrm{Mpc}\right)=1468 h^{-3} \mathrm{Mpc}^{3}$ (see Eq. (D.6)), because the estimate of $\rho$ in Eq. (F.1) requires a weighting function with the comoving distance $r$ as the only variable, whereas the $J_{3}$ weighting function $w(r, s)$ varies with both $r$ and separation $s$; we thus ensure that the weights, and therefore the mean density estimate, are not affected by galaxy clustering. This yields for the 3 spectral-type sub-samples:

$\hat{\rho}=8.47 \times 10^{-3} h^{3} \mathrm{Mpc}^{-3}$ for early - type

$\hat{\rho}=33.58 \times 10^{-3} h^{3} \mathrm{Mpc}^{-3}$ for intermediate - type

$\hat{\rho}=52.41 \times 10^{-3} h^{3} \mathrm{Mpc}^{-3}$ for late - type.

With these values of $\rho, 4 \pi \rho \psi(r) J_{3}(30) \geq 10$ for $r \leq$ $1500 h^{-1} \mathrm{Mpc}$. As a result, $w(r, s) \propto 1 / \psi(r)$, which corresponds to the "equal volume" weighting (Eq. (D.2)).

\section{Appendix G: Projected correlation functions}

Peculiar velocities distort the redshift-space correlation function $\xi(s)$, which then differs from the real-space correlation function $\xi(r)$. In redshift-space, internal random motions within bound structures create the so-called "finger-of-god" structures (elongated along the line-of-sight), while coherent motions on large scales tend to flatten the over-densities along the observer's line of sight (Hawkins et al. 2003). Moreover, the rms uncertainty of $\sim 1.6 h^{-1} \mathrm{Mpc}$ on the line-of-sight separation caused by the redshift measurement uncertainties in the ESS, also contributes to smooth out any clustering in redshift space on scales comparable $s \lesssim 3 h^{-1} \mathrm{Mpc}$ (see Sect. 3.1 and Fig. 4 in Cole et al. 1994).

Because the redshift-space distortions are only radial, one can compute the correlation function as a function of separation parallel $(\pi)$ and perpendicular $\left(r_{\mathrm{p}}\right)$ to the line-of-sight, which allows one to disentangle the effects of peculiar velocities from the genuine spatial correlations. Following the formalism of Fisher et al. (1994), for any two galaxies with redshift positions $\boldsymbol{P}_{1}$ and $\boldsymbol{P}_{2}$, the redshift separation and line-of-sight vectors are defined as $\boldsymbol{S} \equiv \boldsymbol{P}_{2}-\boldsymbol{P}_{1}$ and $\boldsymbol{L} \equiv 0.5 \times\left(\boldsymbol{P}_{1}+\boldsymbol{P}_{2}\right)$, respectively. Therefore, the parallel and perpendicular separations are:

$\pi=\boldsymbol{S} \cdot \boldsymbol{L} /|L|$,

$r_{\mathrm{p}}^{2}=\boldsymbol{S} \cdot \boldsymbol{S}-\pi^{2}$

The redshift-space correlation function $\xi\left(r_{\mathrm{p}}, \pi\right)$ can then be derived for each estimators by replacing $D D(s), R R(s)$ and $D R(s)$ in Eqs. (B.5)-(B.7) with $D D\left(r_{\mathrm{p}}, \pi\right) R R\left(r_{\mathrm{p}}, \pi\right) D R\left(r_{\mathrm{p}}, \pi\right)$, which refer to the data-data, random-random and data-random pair counts resp. at each value of $\left(r_{\mathrm{p}}, \pi\right)$.

In a second stage, $\xi\left(r_{\mathrm{p}}, \pi\right)$ allows one to derive the correlation function $w\left(r_{\mathrm{p}}\right)$ as a function of projected separation $r_{\mathrm{p}}$, which is unaffected by redshift distortions (Davis \& Peebles 1983), and is obtained by integrating $\xi\left(r_{\mathrm{p}}, \pi\right)$ over $\pi$ :

$w\left(r_{\mathrm{p}}\right)=2 \int_{0}^{\infty} \xi\left(r_{\mathrm{p}}, \pi\right) \mathrm{d} \pi=2 \sum_{i} \xi\left(r_{\mathrm{p}}, \pi_{i}\right) \Delta_{i}$,

the summation yields an unbiased estimate of $w\left(r_{\mathrm{p}}\right)$ (Jing et al. 1998), which is related to the real-space correlation function $\xi(r)$ by:

$w\left(r_{\mathrm{p}}\right)=2 \int_{0}^{\infty} \xi\left(\sqrt{r_{\mathrm{p}}^{2}+y^{2}}\right) \mathrm{d} y$.

If $\xi(r)$ is modelled as a power-law $\xi(r)=\left(r / r_{0}\right)^{-\gamma}$, the integral in Eq. (G.3) can be calculated analytically, and yields:

$w\left(r_{\mathrm{p}}\right)=r_{\mathrm{p}}^{1-\gamma} r_{0}^{\gamma} \frac{\Gamma(1 / 2) \Gamma(\gamma / 2-1 / 2)}{\Gamma(\gamma / 2)}$,

where $\Gamma(x)$ is the Gamma function.

The model parameters $r_{0}$ and $\gamma$ in Eq. (G.4) are derived by minimizing the value of $\chi^{2}$ defined as:

$\chi^{2}=\sum_{i} \frac{\left[\xi\left(r_{i}\right)-\left(r_{i} / r_{0}\right)^{-\gamma}\right]^{2}}{\sigma_{i}^{2}}$

where $\xi\left(r_{i}\right)$ and $\sigma_{i}$ are the measured values of the correlation function and its rms fluctuation at a separation $r_{i}$, assuming thereby that the correlation between $\xi\left(r_{i}\right)$ values leads to a small enough bias on the final result. 